\title{
Complex Analytic Construction of the Kuranishi Family on a Normal Strongly Pseudo Convex Manifold
}

\author{
By \\ Takao AKAHORI*
}

\section{Introduction}

Let $(V, x)$ be an analytic subset of a domain in a complex euclidean space with an isolated singular point $x$. Then, we obtain a real submanifold $M$ by cutting the analytic set by a sphere of sufficiently small radius centered at $x$. As is well known $V$ defines a subbundle of fibre dimension $n-1$, to be denoted by ${ }^{\circ} T$, , of the complex tangent bundle CTM of $M$, where $n$ is the dimension of $V$. It is the set of all elements in $C T M$ which are of type $(0,1)$ in $\left.C T V\right|_{M}$. If $V$ is normal and stein, $V$ is completely determined by $\left(M,{ }^{\circ} T^{\prime \prime}\right)($ H. Rossi [6]). So M. Kuranishi considered a deformation theory of isolated singularities $(V, x)$ by means of a deformation theory of pseudo-complex structures $\left(M,{ }^{\circ} T^{\prime \prime}\right)$ (M. Kuranishi [4]). But his result is not definitive enough in the following sense: Since $\bar{\partial}_{b}$ is not elliptic, he had to use the Nash-Moser inverse mapping theorem, which does not preserve the analyticity. So he constructed only a $C^{\infty}$ versal family, without putting a complex structure on the family. We have to take a new approach to remedy this point.

In the previous paper (T. Akahori [1]), the author reformulated an abstract almost pseudo-complex structure sufficiently close to the given one by a tangent bundle valued form $\varphi$ of a certain type and rewrote the integrablity condition as a system of $\bar{\partial}_{b}$-equations. The results are formulated in the following two propositions:

Communicated by S. Nakano, January 25, 1978.

* Department of Mathematics, University of the Ryukyus, Naha 903, Japan. 
Proposition 1.6.1. An almost partially complex structure $T^{\prime \prime}$ at a finite distance from ${ }^{\circ} T^{\prime \prime}$ corresponds to $\varphi$ in $\Gamma\left(M, T^{\prime} \otimes\left({ }^{\circ} T^{\prime \prime}\right)^{*}\right)$ one to one. The following formula determines the correspondence.

$$
{ }^{\varphi} T^{\prime \prime}=\left\{X^{\prime}: X^{\prime}=X+\varphi(X), X \in{ }^{0} T^{\prime \prime}\right\}
$$

Proposition 1.6.2. " $T$ " is integrable if and only if the following relation holds :

$$
P(\varphi)=\bar{\partial}_{T^{\prime}}^{(1)} \varphi+R_{2}(\varphi)+R_{3}(\varphi) .
$$

Here numbers given to propositions refer to those given in later sections.

M. Kuranishi gives the condition equivalent to the above. $\mathrm{He}$ looked for the solutions of corresponding differential equations in $\Gamma\left(M, T^{\prime} \otimes\left({ }^{0} T^{\prime \prime}\right)^{*}\right)$, where he made an essential use of the NashMoser inverse mapping theorem. In this paper we try to look for the solutions of $P(\varphi)=0$ in the subspace $\Gamma\left(M,{ }^{0} \bar{T}^{\prime \prime} \otimes\left({ }^{0} T^{\prime \prime}\right)^{*}\right)$ of $\Gamma\left(M, T^{\prime} \otimes\left({ }^{0} T^{\prime \prime}\right)^{*}\right)$ in case where $M$ is a compact normal strongly pseudo-convex manifold. In $\S 1$, we shall also have the following proposition :

Proposition 1. 7.1. For all elements $\varphi$ in $\Gamma\left(M,{ }^{\circ} \bar{T}^{\prime \prime} \otimes\left({ }^{0} T^{\prime \prime}\right)^{*}\right)$, the relation $P(\varphi)=0$ holds if and only if the following relations hold.

$$
D_{1} \varphi+R_{2}(\varphi)=0
$$

and

$$
L \varphi=0
$$

where $L$ is an operator from $\Gamma\left(M,{ }^{0} \bar{T}^{\prime \prime} \otimes\left({ }^{0} T^{\prime \prime}\right)^{*}\right)$ to $\Gamma\left(M, F \otimes \wedge^{2}\left({ }^{0} T^{\prime \prime}\right)^{*}\right)$, linear over the ring of the $C^{\infty}$-functions and $D_{1}$ is a first order linear differential operator from $\Gamma\left(M,{ }^{\circ} \bar{T}^{\prime \prime} \otimes\left({ }^{0} T^{\prime \prime}\right)^{*}\right)$ to $\Gamma\left(M,{ }^{\circ} \bar{T}^{\prime \prime} \otimes \wedge^{2}\left({ }^{\circ} T^{\prime \prime}\right)^{*}\right)$.

This proposition has nothing to do with normality. Normality appears when we study the first order differential operator $D_{1}$. As was defined by N. Tanaka a compact strongly pseudo-convex manifold 
$M$ is said to be a compact normal strongly pseudo-convex manifold if and only if $M$ has a global real vector field $\xi$ such that $\left[\xi, \Gamma\left(M,{ }^{\circ} T^{\prime \prime}\right)\right] \subset$ $\Gamma\left(M,{ }^{0} T^{\prime \prime}\right)$ and $\xi_{p} \notin R e\left({ }^{0} T_{p}^{\prime \prime} \oplus \bar{T}_{p}^{\prime \prime}\right)$ for every point $P$ of $M$. On a compact normal strongly pseudo-convex manifold $M$ there exists a differential complex of the following form:

$$
0 \rightarrow \Gamma\left(M,{ }^{0} \bar{T}^{\prime \prime}\right) \stackrel{{ }^{D}}{\longrightarrow} \Gamma\left(M,{ }^{0} \bar{T}^{\prime \prime} \otimes\left({ }^{0} T^{\prime \prime}\right) *\right) \stackrel{{ }^{D_{1}}}{\longrightarrow} \Gamma\left(M,{ }^{0} \bar{T}^{\prime \prime} \otimes{ }^{2}\left({ }^{0} T^{\prime \prime}\right)^{*}\right) .
$$

Using the above fact, we shall solve the differential equation

$$
D_{1} \varphi+R_{2}(\varphi)=0
$$

and

$$
L \varphi=0
$$

in $\Gamma\left(M .{ }^{\circ} \bar{T}^{\prime \prime} \otimes\left({ }^{0} T^{\prime \prime}\right)^{*}\right)$. The following propositions assure that we can construct a versal family likewise in the case of compact complex manifold.

Proposition 3.13. Assuming that $(M, \xi)$ is a compact normal strongly pseudo-convex manifold and $\operatorname{dim}_{R} M=2 n-1 \geq 7$, the following relation holds.

$$
\left\|N D_{1}^{*} R_{2}(\varphi)\right\|_{(m)}^{\prime \prime}<\|\varphi\|_{(m)}^{\prime \prime 2}
$$

uniformly for all $\varphi$ in $\Gamma\left(M,{ }^{\circ} \bar{T}^{\prime \prime} \otimes\left({ }^{\circ} T^{\prime \prime}\right) *\right)$, where $m$ is a non-negative integer. (The author got the idea of this estimate through the communication with M. Kuranishi.)

Proposition 4. 1. Denote by $\Gamma_{(m)}^{\prime \prime}\left(M,{ }^{0} \bar{T}^{\prime \prime} \otimes\left({ }^{0} T^{\prime \prime}\right)^{*}\right)\left(\operatorname{resp} . \Gamma_{(m)}^{\prime \prime}(M, F \otimes\right.$ $\left.\left.{ }^{2} \wedge\left({ }^{0} T^{\prime \prime}\right)^{*}\right)\right)$ the Hilbert space obtained by completing $\Gamma\left(M,{ }^{\circ} \bar{T}^{\prime \prime} \otimes\left({ }^{0} T^{\prime \prime}\right)^{*}\right)$ (resp. $\Gamma\left(M, F \otimes \wedge^{2}\left({ }^{0} T^{\prime \prime}\right)^{*}\right)$ ) with respect to the norm \|\|$_{(m)}^{\prime \prime}$ introduced in § 3. Setting $Z_{D_{1}}=\left\{\varphi: \varphi=\left(H+D D^{*} N\right) \varphi\right.$, $\varphi$ in $\left.\Gamma_{(m)}^{\prime \prime}\left(M,{ }^{\circ} \bar{T}^{\prime \prime} \otimes\left({ }^{0} T^{\prime \prime}\right)^{*}\right)\right\}$, $L Z_{D_{1}}$ is closed in $\Gamma_{(m)}^{\prime \prime}\left(M, \mathrm{~F} \otimes \wedge^{2}\left({ }^{0} T^{\prime \prime}\right)^{*}\right)$.

Then we have the main Theorem in this paper.

Main Theorem 5. 2. Under the assumptions that $H_{T}^{(2)}=0, \operatorname{dim}_{R} M$ $=2 n-1 \geq 7$ and $(M, \xi)$ is a compact normal strongly pseudo-convex 
manifold, there exists a deformation $\varphi(t)$, complex analytically parametrized by a neighborhood of the origin in the euclidean space $\mathscr{H}$ such that:

1) There exists an element $\varphi(t)$, a $\Gamma\left(M,{ }^{\circ} \bar{T}^{\prime \prime} \otimes\left({ }^{\circ} T^{\prime \prime}\right)^{*}\right)$ valued function of $t$ of $C^{2}$-class such that the following relation holds:

$$
\varphi(0)=0
$$

and

$$
P(\varphi(t))=0
$$

2) The linear term of $\varphi\left(t_{1}, t_{2}, \ldots, t_{q}\right)$ is $\sum_{\lambda=1}^{q} \beta_{\lambda} t_{\lambda}$, where $\left\{\beta_{\lambda}\right\}_{1 \leq k \leq q}$ is a system of basis of $\mathscr{H}$ and $\operatorname{dim} \mathscr{H}=q$.

In $\S 6$, we prove that this family is the versal family of isolated singularities in the sense of $\mathrm{M}$. Kuranishi.

The assumption of normality of $M$ is not too restrictive, because Tanaka has proved that the isolated singularity $(V, x)$ defined by a quasihomogeneous polynomial in a euclidean space has a compact normal strongly pseudo-convex manifold $M$ such that $M=\partial V^{\prime}$, embedded in a slightly larger open manifold $V$.

It is noted that $H$. Gravert and A. Douady constructed a versal family of isolated singularities in another way. We hope that this method gives a new insight into the problems of deformation of singularities (not necessarily isolated).

\section{§. The Boundary of a Complex Analytic Space and Its Deformation}

(1.1) In this section we shall study the boundary of a complex analytic space and recall its deformation theory developed in [1]. Let $Y^{\prime}$ be a complex analytic space of complex dimension $n$. Let $Y$ be a relatively compact open subset of $Y^{\prime}$ with strongly pseudo-convex smooth boundary $b Y$. This means that in a neighbourhood of $b Y$, we can find a complex coordinate system $\left(z_{1}, z_{2}, \ldots, z_{n}\right)$ and a real valued $C^{\infty}$ function $r$ with the following properties : $r<0$ in $Y, r>0$ 
outside of $\bar{Y}, d r \neq 0$ on $b Y$ and $\left\{r_{z_{i} \bar{z}_{j}}\right\}$ is a positive definite hermitian matrix on $b Y$. Then $Y$ defines a subbundle of fiber dimension $n-1$, say ' $T$ ", of the complex tangent bundle $C T b Y$ of $b Y$, where $n$ is the dimension of $Y$. We call the subbundle obtained as above a partially complex structure. It is the set of all elements in $C T b Y$ which are of type $(0,1)$ in $Y^{\prime}$, i. e., using the notation ${ }^{\circ} T^{\prime \prime}$, an element of ${ }^{\circ} T^{\prime \prime}$ is of the form

$$
\sum_{k=1}^{n} a_{k}(x) \partial / \partial \bar{z}_{k}
$$

where $x$ in $b Y$ and we require that

$$
\sum_{k=1}^{n} a_{k}(x) \partial r / \partial \bar{z}_{k}=0
$$

on $b Y$.

(1.2) J. J. Kohn has shown that the methods of harmonic integrals work on such boundaries and describes the $\bar{\partial}_{b}$-equations. Thus we can bring the techniques of harmonic integrals into the study of isolated singularities. To do this we study the abstract strongly pseudo-convex manifold owing to Tanaka.

Let $M$ be a differentiable manifold. By a partially complex structure on $M$, we mean a pair $\left(M,{ }^{\circ} T^{\prime \prime}\right)$ of $M$ and a subbundle ${ }^{\circ} T^{\prime \prime}$ of $C T M$, where ${ }^{\circ} T$ " satisfies the following conditions A. 1) and A. 2):

A. 1) ${ }^{\circ} T^{\prime \prime} \cap{ }^{0} \bar{T}^{\prime \prime}=0$,

A. 2) for any $X, Y$ in $\Gamma\left(M,{ }^{\circ} T^{\prime \prime}\right),[X, Y]$ is in $\Gamma\left(M,{ }^{\circ} T^{\prime \prime}\right)$.

$M$ with ${ }^{\circ} T$ " is also called a partially complex manifold. Let $M$ be a partially complex manifold. Then there exists the following exact sequence of vector bundles.

$$
0 \rightarrow{ }^{0} T^{\prime \prime} \oplus^{0} \bar{T}^{\prime \prime} \rightarrow C T M \rightarrow C T M /{ }^{\circ} T^{\prime \prime} \oplus^{\circ} \bar{T}^{\prime \prime} \rightarrow 0 .
$$

Differentiably, this sequence splits and the splitting commutes with the operation of complex conjugation. So there exists, differentiable vector bundle isomorphism

$$
\mu:{ }^{\circ} T^{\prime \prime} \oplus{ }^{0} \bar{T}^{\prime \prime} \oplus C T M /{ }^{\circ} T^{\prime \prime} \oplus{ }^{\circ} \bar{T}^{\prime \prime} \rightarrow C T M .
$$


We shall fix this splitting $C T M={ }^{\circ} T^{\prime \prime} \oplus^{\circ} T^{\prime \prime} \oplus F$, where $F=\mu(C T M$ / $\left.{ }^{\circ} T^{\prime \prime} \oplus^{\circ} T^{\prime \prime}\right)$. For each $x$ in $M$, we define $\mathrm{F}$-valued hermitian form $L_{x}$ by

$$
L_{x}(X, Y)=\sqrt{-1}[X, \bar{Y}]_{F}
$$

for $X, Y$ in $\Gamma\left(M,{ }^{\circ} T^{\prime \prime}\right)$, where by $[X, \bar{Y}]_{F}$ we denote the projection of $[X, \bar{Y}]$ to $F$ according to the above splitting $C T M={ }^{\circ} T^{\prime \prime} \oplus^{\circ} \bar{T}^{\prime \prime} \oplus F$. The hermitian form $L_{x}$ is usually called the Levi form at $x$. We say that $\left(M,{ }^{\circ} T^{\prime \prime}\right)$ is a strongly pseudo-convex manifold (abbreviation: an s. p. c. manifold) if $\operatorname{dim} F=1$ and if the Levi form $L_{x}$ is definite at each $x$, i. e., the conditions $x$ in ${ }^{\circ} T^{\prime \prime}$ and $[X, \bar{X}]_{F}=0$ imply $X=0$. If there exists a complex analytic space $Y$ and its boundary $b Y$ such that $b Y$ is strongly pseudo-convex, then the Levi form $L_{x}$ is definite at each $x$. Conversely if the Levi form $L_{x}$ is definite at each $x$ and $Y b Y$ is compact. $b Y$ is strongly pseudo-convex in $Y$. It is a theorem of $\mathrm{H}$. Rossi that if $Y$ is normal and stein, $Y$ is determined by the pair $\left(b Y,{ }^{\circ} T^{\prime \prime}\right)$. (H. Rossi [6].)

(1.3) In this paper, we shall study the deformation of partially complex structures on a normal s. p. c. manifold and construct the Kuranishi family on it. To do this, we shall recall the definition of a normal s. p. c. manifold.

Definition 1. 3.1. An s.p.c. manifold $\left(M,{ }^{\circ} T^{\prime \prime}\right)$ is called a normal s.p.c. manifold if and only if there exists a global real vector field $\xi$ on $M$ such that

$\left[\xi, \Gamma\left(M,{ }^{0} T^{\prime \prime}\right)\right] \subset \Gamma\left(M,{ }^{0} T^{\prime \prime}\right), \xi_{p} \notin \operatorname{Re}\left({ }^{0} T_{p}^{\prime \prime} \oplus^{0} \bar{T}_{p}^{\prime \prime}\right)$ for any $p$ of $M$.

Example. A typical example of a normal s. p.c. manifold is a Brieskorn variety. Let $f\left(z_{1}, z_{2}, \ldots, z_{n}\right)$ be a weighted homogeneous polynomial of type $\left(a_{1}, a_{2}, \ldots, a_{n}\right)$, where $a_{1}, a_{2}, \ldots, a_{n}$ are positive rational numbers (Milnor [5]). By definition the polynomial $f$ satisfies the equality

$$
f\left(e^{c / a_{1}} z_{1}, \ldots, e^{c / a_{n}} z_{n}\right)=e^{c} f\left(z_{1}, \ldots, z_{n}\right)
$$

for every complex number $c$. Clearly we have $f(0)=0$. We assume 
that the origin $o$ is an isolated singular point of $f$. It is easy to see that the origin is the only isolated singular point of $f$. We put $Y=f^{-1}(0)$, and for every positive number $\varepsilon$ we denote by $M_{\varepsilon}$ the intersection of the complex hypersurface $f^{-1}(0)$ with the sphere $S^{2 n-1}(\varepsilon)$. Then $M$ is an s. p. c. real hypersurface of $Y$. We shall show that this s.p.c. manifold $M$ is normal. Define a one parameter group of holomorphic transformations of $C^{n}, \tau_{t}$, by

$$
\tau_{t}(z)=\left(z_{1}^{\prime}, \ldots, z_{n}^{\prime}\right), z_{i}^{\prime}=e^{t \sqrt{-1 /} a_{i}} z_{i} \text {. }
$$

Clearly $\tau_{t}$ leaves invariant $Y, S^{2 n-1}(\varepsilon)$ and hence $M$. Let $\xi$ be a vector field on $M$ induced by the one parameter group. Then $\xi$ is a real vector field and satisfies the relation

$$
\left[\xi, \Gamma\left(M,{ }^{\circ} T^{\prime \prime}\right)\right] \subset \Gamma\left(M,{ }^{\circ} T^{\prime \prime}\right),
$$

i. e., $\xi$ is the real part of a holomorphic vector field and $\xi_{p} \notin \operatorname{Re}\left({ }^{0} \bar{T}_{p}^{\prime \prime} \oplus\right.$ ${ }^{\circ} T_{p}^{\prime \prime}$ ) for every point $p$ on $M$.

(1.4) Let $(M, \xi)$ be a normal s. p. c. manifold. Then there exists the following bundle isomorphism.

$$
\mu:{ }^{\circ} \bar{T}^{\prime \prime} \oplus{ }^{0} T^{\prime \prime} \oplus C \xi \stackrel{\longrightarrow}{\longrightarrow} C T M .
$$

We shall fix the splitting $C T M={ }^{\circ} \bar{T}^{\prime \prime} \oplus{ }^{\circ} T^{\prime \prime} \oplus F$ newly, where $F=\mu(C \xi)$. Using this splitting, we define a first order differential operator

$$
D: \Gamma\left(M,{ }^{0} \bar{T}^{\prime \prime}\right) \rightarrow \Gamma\left(M,{ }^{\circ} \bar{T}^{\prime \prime} \otimes\left({ }^{0} \bar{T}^{\prime \prime}\right)^{*}\right)
$$

by

$$
\operatorname{Du}(X)=[X, u]_{0_{T^{\prime \prime}}}
$$

for all $u$ in $\Gamma\left(M,{ }^{\circ} \bar{T}^{\prime \prime}\right)$ and $X$ in $\Gamma\left(M,{ }^{\circ} T^{\prime \prime}\right)$. This map is well defined since the relation. $[f X, u]_{0_{\bar{T}^{\prime \prime}}}=f[X, u]_{0_{\bar{T}^{\prime \prime}}}$ holds for all $C^{\infty}$ functions $f, X$ in $\Gamma\left(M,{ }^{\circ} T^{\prime \prime}\right)$ and $u$ in $\Gamma\left(M,{ }^{\circ} \bar{T}^{\prime \prime}\right)$. Here by $[X, u]_{0_{\bar{T}^{\prime \prime \prime}}}$, we denote the projection of $[X, u]$ to ${ }^{\circ} \bar{T}^{\prime \prime}$ according to the above new splitting $C T M={ }^{\circ} \bar{T}^{\prime \prime} \oplus{ }^{0} T^{\prime \prime} \oplus F$. Then we have

B. 1) $X(f u)=X f \cdot u+f X u$,

B. 2) $[X, Y] u=X(Y u)-Y(X u)$, 
where $u$ in $\Gamma\left(M,{ }^{\circ} \bar{T}^{\prime \prime}\right), f$ in $\Gamma(M, C), X, Y$ in $\Gamma\left(M,{ }^{\circ} T^{\prime \prime}\right)$ and we put $X u=D u(X)=[X, u]_{0_{\tilde{T}},}$.

In fact, from the relation $[X, f u]=(X f) u+f[X, u]$, we have

$$
[X, f u]_{0_{T^{\prime \prime}}}=(X f) u+f[X, u]_{0_{T^{\prime \prime}}} \text {. }
$$

So the relation $\mathrm{B} .1)$ is proven. From the Jacobi identity $[[X, Y], u]=$ $[X,[Y, u]]-[Y,[X, u]]$, we have the relation $[[X, Y], u]_{0_{x^{\prime \prime}}}=$ $[X,[Y, u]]_{0_{T^{\prime \prime}}}-[Y,[X, u]]_{0_{T^{\prime \prime}}}$ According to the new splitting, we have the relation

$$
[X, u]=[X, u]_{0_{T^{\prime \prime}}}+[X, u]_{0_{T^{\prime \prime}}}+[X, u]_{F}
$$

and

$$
[Y, u]=[Y, u]_{0_{T^{\prime \prime}}}+[Y, u]_{0_{T^{\prime \prime}}}+[Y, u]_{F}
$$

So we have

$$
\begin{aligned}
{[[x, Y], u]_{0_{T^{\prime \prime}}}=} & {[X,[Y, u]]_{0_{T^{\prime \prime}}}-[Y,[X, u]]_{0_{T^{\prime \prime}}} } \\
= & {\left[X,[Y, u]_{0_{T^{\prime \prime}}}+[Y, u]_{0_{T^{\prime \prime}}}+[Y, u]_{F}\right]_{0_{T^{\prime \prime}}} } \\
& -\left[Y,[X, u]_{0_{T^{\prime \prime}}}+[X, u]_{0_{T^{\prime \prime}}}+[X, u]_{F}\right]_{0_{T^{\prime \prime}}} \\
= & {\left[X,[Y, u]_{0_{T^{\prime \prime}}}\right]_{0_{T^{\prime \prime}}}-\left[Y,[X, u]_{0_{T^{\prime \prime}}}\right]_{0_{T^{\prime \prime}}} }
\end{aligned}
$$

(From the relations $\left[\xi, \Gamma\left(M,{ }^{\circ} T^{\prime \prime}\right)\right] \subset \Gamma\left(M,{ }^{\circ} T^{\prime \prime}\right)$ and $\left[\Gamma\left(M,{ }^{\circ} T^{\prime \prime}\right)\right.$, $\left.\Gamma\left(M,{ }^{\circ} T^{\prime \prime}\right)\right] \subset \Gamma\left(M,{ }^{\circ} T^{\prime \prime}\right)$. $)$ The relation B. 2) is proven.

(1.5) From the relation B.1) and B.2), we can define an operator $D_{p}$ for each $p \geq 1$ from $\Gamma\left(M,{ }^{\circ} \bar{T}^{\prime \prime} \otimes \wedge\left({ }^{\circ} T^{\prime \prime}\right)^{*}\right)$ to $\Gamma\left(M,{ }^{\circ} \bar{T}^{\prime \prime} \otimes\right.$ $\left.\bigwedge^{p+1}\left({ }^{0} T^{\prime \prime}\right)^{*}\right)$ as follows. For any $\varphi$ in $\Gamma\left(M,{ }^{\circ} \bar{T}^{\prime \prime} \otimes \bigwedge^{p}\left({ }^{0} T^{\prime \prime}\right)^{*}\right)$, we set

$$
\begin{aligned}
& D_{p} \varphi\left(X_{1}, X_{2}, \ldots, X_{p+1}\right)=\sum_{i}(-1)^{i+1} X_{i} \bullet \varphi\left(X_{1}, \ldots, \check{X}_{i}, \ldots, X_{p+1}\right) \\
& \quad+\sum_{i<j}(-1)^{i+j} \varphi\left(\left[X_{i}, X_{j}\right], \ldots, \check{X}_{i}, \ldots, \check{X}_{j}, \ldots, X_{p+1}\right)
\end{aligned}
$$

where $X_{i}$ are in $\Gamma\left(M,{ }^{\circ} T^{\prime \prime}\right)$ and we put $X u=D u(X)=[X, u]_{0_{T^{\prime \prime}}}$.

Then we have the following differential complex.

$$
\begin{aligned}
& 0 \longrightarrow \Gamma\left(M,{ }^{0} \bar{T}^{\prime \prime}\right) \underset{D^{\prime}}{\longrightarrow} \Gamma\left(M,{ }^{\circ} \bar{T}^{\prime \prime} \otimes\left({ }^{0} T^{\prime \prime}\right)^{*}\right) \underset{D_{1}}{\longrightarrow} \Gamma\left(\mathrm{M},{ }^{0} \bar{T}^{\prime \prime} \otimes \bigwedge^{2}\left({ }^{0} T^{\prime \prime}\right)^{*}\right) \\
& \longrightarrow \Gamma\left(M,{ }^{0} \bar{T}^{\prime \prime} \otimes \wedge^{p}\left({ }^{0} T^{\prime \prime}\right) *\right) \underset{D_{p}}{\longrightarrow} \Gamma\left(M,{ }^{\circ} \bar{T}^{\prime \prime} \otimes \wedge^{p+1}\left({ }^{0} T^{\prime \prime}\right)^{*}\right) \longrightarrow
\end{aligned}
$$


Especially we have

$$
D_{1} \varphi(X, Y)=[\varphi(X), Y]_{0_{T^{\prime \prime}}}+[X, \varphi(Y)]_{0_{\tilde{T}^{\prime \prime}}}-\varphi([X, Y]) .
$$

(1.6) Now we shall recall results obtained in [1]. Let $\left(M,{ }^{\circ} T^{\prime \prime}\right)$ be an abstract s. p. c. manifolds. Then we have

Proposition 1.6. 1. Setting $T^{\prime}={ }^{\circ} \bar{T}^{\prime \prime} \oplus F$, an almost partially complex structure " $T$ " at a finite distance from ' ${ }^{\circ} T^{\prime}$ corresponds to $\varphi$ in $\Gamma\left(M, T^{\prime} \otimes\left({ }^{0} T^{\prime \prime}\right)^{*}\right)$ bijectively. The following formula determines a bijective correspondence.

$$
{ }^{\varphi} T^{\prime \prime}=\left\{X+\varphi(X): X \in{ }^{\circ} T^{\prime \prime}\right\}
$$

Proposition 1.6. 2. " $T$ " is integrable if and only if satisfies the following relation.

$$
P(\varphi)=\bar{\partial}_{T^{\prime}}^{(1)} \varphi+R_{2}(\varphi)+R_{3}(\varphi)=0,
$$

where

$$
R_{2}(\varphi)(X, Y)=[\varphi(X), \varphi(Y)]_{T^{\prime}}-\varphi\left([X, \varphi(Y)]_{0_{T^{\prime \prime}}}+[\varphi(X), Y]_{0_{T^{\prime \prime}}}\right)
$$

and

$$
R_{3}(\varphi)(X, Y)=-\varphi\left([\varphi(X), \varphi(Y)]_{T^{\prime \prime}}\right) .
$$

For the proof, see Proposition 1.1 and Proposition 2. 1 in [1].

(1.7) We shall solve this first order non-linear differential equation $p(\varphi)=0$. But it is difficult to solve this in $\Gamma\left(M, T^{\prime} \otimes\left({ }^{\circ} T^{\prime \prime}\right)^{*}\right)$ since $\breve{\partial}_{T^{\prime}}$ is not elliptic. We shall look for the solution of $p(\varphi)=0$ on a restricted space.

Proposition 1. 7. 3. $P(\varphi)=0$ holds for all $\varphi$ in $\Gamma\left(M,{ }^{\circ} \bar{T}^{\prime \prime} \otimes\left({ }^{\circ} T^{\prime \prime}\right)^{*}\right)$ if and only if $D_{1} \varphi+R_{2}(\varphi)=0$ and $L=0$, where we put $L \varphi(X, Y)=$ $[\varphi(X), Y]_{F}+[X, \varphi(Y)]_{F}$.

Proof. The relation $P(\varphi)=0$ holds if and only if $(P(\varphi))_{0_{T^{\prime \prime}}}=0$ and $(P(\varphi))_{F}=0$, where by $(P(\varphi))_{0_{T^{\prime \prime}}}$ we denote the projection from $\Gamma\left(M, T^{\prime} \otimes \wedge^{2}\left({ }^{0} T^{\prime \prime}\right)^{*}\right)$ to $\Gamma\left(M,{ }^{0} \bar{T}^{\prime \prime} \otimes \wedge^{2}\left({ }^{0} T^{\prime \prime}\right) *\right)$ according to the splitting 
$C T M={ }^{\circ} T^{\prime \prime} \oplus^{\circ} \bar{T}^{\prime \prime} \oplus F$ and by $(P(\varphi))_{F}$ we denote the projection from $\Gamma\left(M, T^{\prime} \otimes \wedge^{2}\left({ }^{0} T^{\prime \prime}\right)^{*}\right)$ to $\Gamma\left(M, F \otimes \bigwedge^{2}\left({ }^{0} T^{\prime \prime}\right)^{*}\right)$ according to the splitting $C T M={ }^{\circ} T^{\prime \prime} \oplus^{\circ} \bar{T}^{\prime \prime} \oplus F$. We shall compute the relations $(P(\varphi))_{0_{T^{\prime \prime}}}=0$ and $(P(\varphi))_{F}=0$ for $\varphi$ in $\Gamma\left(M,{ }^{\circ} \bar{T}^{\prime \prime} \otimes\left({ }^{0} T^{\prime \prime}\right)^{*}\right)$.

$$
\begin{aligned}
(P(\varphi))_{0_{T^{\prime \prime}}}(X, Y) & =\left([\varphi(X), Y]_{T^{\prime}}+[X, \varphi(Y)]_{T^{\prime}}-\varphi([X, Y])\right. \\
& +[\varphi(X), \varphi(Y)]_{T^{\prime}}-\varphi\left([\varphi(X), Y]_{0_{T^{\prime \prime}}}\right. \\
& \left.+[X, \varphi(Y)]_{0_{T^{\prime \prime}}}\right)+\varphi([\varphi(X), \varphi(Y)])_{0_{T^{\prime \prime}}} \\
& =D_{1} \varphi(X, Y)+R_{2}(\varphi)(X, Y) \\
(P(\varphi))_{F}(X, Y) & =\left([\varphi(X), Y]_{T^{\prime}}+[X, \varphi(Y)]_{T^{\prime}}-\varphi([X, Y])\right)_{F} \\
& =L \varphi(X, Y) .
\end{aligned}
$$

So Proposition 1.7.3 is proven.

Q. E. D.

\section{§ 2. The Complex $\left(\mathbb{I}\left(M, \mathbb{F} \otimes \bigwedge^{p}\left({ }^{0} \mathbb{T}^{\prime \prime}\right)^{*}\right), \bar{\partial}_{F}^{(p)}\right)$}

(2.1) In this section we shall define a differential complex $\left(\Gamma\left(M, F \otimes \wedge\left({ }^{0} T^{\prime \prime}\right)^{*}\right), \bar{\partial}_{F}^{(p)}\right)$. By $\Gamma\left(M, F \otimes \wedge^{p}\left({ }^{0} T^{\prime \prime}\right)^{*}\right)$ we denote the following

$$
\Gamma\left(M, F \otimes \stackrel{\wedge}{\wedge}\left({ }^{0} T^{\prime \prime}\right)^{*}\right)=\left\{\varphi: \varphi=\psi \otimes \xi, \psi \in \Gamma\left(M, \otimes \bigwedge^{p}\left({ }^{0} T^{\prime \prime}\right)^{*}\right)\right\} .
$$

We define a first order differential operator $\bar{\partial}_{F}$ from $\Gamma(M, F)$ to $\Gamma\left(M, F \otimes\left({ }^{\circ} T^{\prime \prime}\right)^{*}\right)$ as follows : For any element $u \Gamma(M, F)$ we set

$$
\bar{\partial}_{F} u(X)=[X, u]_{F},
$$

for $X$ in $\Gamma\left(M,{ }^{\circ} T^{\prime \prime}\right)$, where by $[X, u]_{F}$ we denote the projection of $[X, u]$ to $F$ according to the splitting $C T M={ }^{\circ} T^{\prime \prime} \oplus^{\circ} \bar{T}^{\prime \prime} \oplus F$ introduced in $\S 1$.

(2.2) Then we get the following relations C. 1) and C. 2).

C. 1) $X(f u)=X f \cdot u+f X u$,

C. 2) $[X, Y] u=X(Y u)-Y(X u)$,

where $u$ is in $\Gamma(M, F), f$ is a $C^{\infty}$ function on $M, X, Y$ are in $\Gamma\left(M,{ }^{\circ} T^{\prime \prime}\right)$ and we put $X u=\bar{\partial}_{F} u(X)=[X, u]_{F}$. 
Proof. In fact from the relation $[X, f u]=X f \cdot u+f[X, u]$, we have

$$
[X, f u]_{F}=X f u+f[X, u]_{F} .
$$

So we get the relation C. 1). From the Jacobi identity, we have

$$
[[X, Y], u]_{F}=[X,[Y, u]]_{F}-[Y,[X, u]]_{F} .
$$

(M. $\xi$ ) being a normal s. p. c. manifold, we have the relation

$$
[Y, u]=[Y, u]_{F}+[Y, u]_{0^{\prime \prime \prime}}
$$

for all $Y$ in $\Gamma\left(M,{ }^{\circ} T^{\prime \prime}\right)$ and $u$ in $\Gamma(M, F)$. (In fact for each $u$ in $\Gamma(M, F)$ there exists a $C^{\infty}$ function $u^{\prime}$ on $M$ such that

$$
u=u^{\prime} \xi \text {. }
$$

Therefore we have the relation

$$
\begin{aligned}
{[Y, u] } & =\left[Y, u^{\prime} \xi\right] \\
& =Y u^{\prime} \cdot \xi+u^{\prime}[Y, \xi] \\
& =[Y, u]_{F}+[Y, u]_{T_{T^{\prime \prime}}}
\end{aligned}
$$

because the relation $\left[\Gamma\left(M,{ }^{\circ} T^{\prime \prime}\right), \xi\right] \subset \Gamma\left(M,{ }^{\circ} T^{\prime \prime}\right)$ holds. $)$ So we have the following relation:

$$
\begin{aligned}
{[[X, Y], u]_{F}=} & {[X,[Y, u]]_{F}-[Y,[X, u]]_{F} } \\
= & {\left[X,[Y, u]_{F}+[Y, u]_{T^{\prime \prime}}\right]_{F}-\left[Y,[X, u]_{F}\right.} \\
& \left.+[X, u]_{0^{\prime \prime}}\right]_{F} \\
= & {\left[X,[Y, u]_{F}\right]_{F}-\left[Y,[X, u]_{F}\right]_{F}, }
\end{aligned}
$$

for all $X, Y$ in $\Gamma\left(M,{ }^{\circ} T^{\prime \prime}\right)$.

So we have the relation C. 2).

(2. 3) From C. 1) and C. 2), we can define a first order differential operator $\bar{\partial}_{F}^{(p)}$ from $\Gamma\left(M, F \otimes \wedge^{p}\left({ }^{0} T^{\prime \prime}\right)^{*}\right)$ to $\Gamma\left(M, F \otimes \bigwedge^{p+1}\left({ }^{0} T^{\prime \prime}\right)^{*}\right)$ as follows : for any $\phi$ in $\Gamma\left(M, F \otimes \wedge^{p}\left({ }^{0} T^{\prime \prime}\right)^{*}\right)$,

$$
\begin{aligned}
& \bar{\partial}_{F}^{(p)} \psi\left(X_{1}, \ldots, X_{p+1}\right)= \sum_{i=1}^{p+1}(-1)^{i+1} X_{i} \bullet \psi\left(X_{1}, \ldots, \check{X}_{i}, \ldots, X_{p+1}\right) \\
&+\sum_{i<j}(-1)^{i+j} \psi\left(\left[X_{i}, X_{j}\right], X_{1}, \ldots, \check{X}_{i}, \ldots,\right. \\
&\left.\check{X}_{j}, \ldots, X_{p+1}\right),
\end{aligned}
$$


where $X_{i}^{\prime}$ 's are in $\Gamma\left(M,{ }^{\circ} T^{\prime \prime}\right)$ and we put

$$
X_{i} \cdot \psi\left(X_{1}, \ldots, \check{X}_{i}, \ldots, X_{p+1}\right)=\bar{\partial}_{F}\left(\psi\left(X_{1}, \ldots, \check{X}_{i}, \ldots, X_{p+1}\right)\left(X_{i}\right)\right) .
$$

Then we have the following differential complex

$$
\begin{aligned}
0 \rightarrow \Gamma(M, F) & \stackrel{\bar{\partial}_{F}}{\longrightarrow} \Gamma\left(M, F \otimes\left({ }^{0} T^{\prime \prime}\right)^{*}\right) \stackrel{\bar{\partial}_{F}^{(1)}}{\longrightarrow} \Gamma\left(M, F \otimes \bigwedge^{2}\left({ }^{0} T^{\prime \prime}\right)^{*}\right) \\
& \stackrel{\bar{\partial}_{F}^{(p-1)}}{\longrightarrow} \Gamma\left(M, F \otimes \wedge\left({ }^{0} T^{\prime \prime}\right)^{*}\right) \stackrel{\bar{\partial}_{F}^{(p)}}{\longrightarrow} \Gamma\left(M, F \otimes \bigwedge^{p+1}\left({ }^{0} T^{\prime \prime}\right)^{*}\right)
\end{aligned}
$$

(2.4) We shall study the $\bar{\partial}_{F}^{(p)}$-cohomology group. We set a linear map $\tau_{p}$ from $\Gamma\left(M, \stackrel{p}{\wedge}\left({ }^{0} T^{\prime \prime}\right)^{*}\right)$ to $\Gamma\left(M, F \otimes \wedge\left({ }^{0} T^{\prime \prime}\right)^{*}\right)$ as follows : For each $\psi$ in $\Gamma\left(M, \stackrel{p}{\wedge}\left({ }^{0} T^{\prime \prime}\right)^{*}\right)$, we set

$$
\tau_{p}(\psi)=\psi \otimes \xi .
$$

Then the following diagram commutes:

$$
\begin{array}{cc}
\Gamma\left(M, \stackrel{p}{\wedge}\left({ }^{0} T^{\prime \prime}\right)^{*}\right) & \stackrel{\bar{\partial}_{b}^{(p)}}{\longrightarrow} \Gamma\left(M,{\left.\stackrel{p}{\wedge+1}\left({ }^{0} T^{\prime \prime}\right)^{*}\right)}^{\longrightarrow \tau_{p}}\right. \\
\Gamma\left(M, F \otimes \stackrel{\tau_{p+1}}{\wedge}\left({ }^{0} T^{\prime \prime}\right)^{*}\right) \stackrel{\bar{\partial}_{F}^{(p)}}{\longrightarrow} \Gamma\left(M, F \otimes \bigwedge^{p+1}\left({ }^{0} T^{\prime \prime}\right)^{*}\right) .
\end{array}
$$

(In fact for any $\phi$ in $\Gamma\left(M, \stackrel{p}{\wedge}\left({ }^{0} T^{\prime \prime}\right)^{*}\right)$, we have

$$
\begin{aligned}
\bar{\partial}_{F}^{(p)} & (\psi \otimes \xi)\left(X_{1}, X_{2}, \ldots, X_{p+1}\right) \\
= & \sum_{j}(-1)^{j+1}\left[X, \psi\left(X_{1}, X_{2}, \ldots, \check{X}_{j}, \ldots, X_{p+1}\right) \xi\right]_{F} \\
& +\sum_{i<j}(-1)^{i+j} \psi\left(\left[X_{i}, X_{j}\right], \ldots, \check{X}_{i}, \ldots, \check{X}_{j}, \ldots, X_{p+1}\right) \xi \\
= & \sum_{j}(-1)^{j+1} X_{\jmath} \psi\left(X_{1}, X_{2}, \ldots, \check{X}_{j}, \ldots, X_{p+1}\right) \xi \\
& +\sum_{i<j}(-1)^{i+j} \psi\left(\left[X_{i}, X_{j}\right], \ldots, \check{X}_{i}, \ldots, \check{X}_{j}, \ldots, X_{p+1}\right) \xi \\
= & \left(\bar{\partial}_{b}^{(p)} \psi\right)\left(X_{1}, X_{2}, \ldots, X_{p+1}\right) \xi \\
= & \left(\bar{\partial}_{b}^{(p)} \psi \otimes \xi\right)\left(X_{1}, X_{2}, \ldots, X_{p+1}\right),
\end{aligned}
$$

for any $X_{i}$ in $\Gamma\left(M,{ }^{\circ} T^{\prime \prime}\right)$. For the details see T. Akahori [2].)

(2.5) Under this situation, we have the following Proposition 2.5. 1 .

Proposition 2.5.1. The map $\tau_{p}$ induces the following isomorphism: 
$\operatorname{Ker} \bar{\partial}_{b}^{(p)} / \operatorname{Im} \bar{\partial}_{b}^{(p-1)} \longrightarrow \operatorname{Ker} \bar{\partial}_{F}^{(p)} / \operatorname{Im} \bar{\partial}_{F}^{(p-1)}$.

Proof. From the fact that $\tau_{p}$ is an isomorphism from $\Gamma(M, \stackrel{p}{\wedge}$ $\left.\left({ }^{0} T^{\prime \prime}\right)^{*}\right)$ to $\Gamma\left(M, F \otimes \stackrel{\wedge}{\wedge}\left({ }^{0} T^{\prime \prime}\right)^{*}\right)$ and from the relation $\bar{\partial}_{F}^{(p)} \cdot \tau_{p}=\tau_{p+1} \cdot \bar{\partial}_{b}^{(p)}$, we have our Proposition.

Q. E. D.

(2.6) Now we shall introduce a first order derivation $L_{\xi}$ on $\Gamma\left(M, F \otimes \stackrel{p}{\wedge}\left({ }^{0} T^{\prime \prime}\right)^{*}\right)$. For each $u=u^{\prime} \otimes \xi$ in $\Gamma\left(M, F \otimes \wedge\left({ }^{0} T^{\prime \prime}\right)^{*}\right)$ with $u^{\prime}$ in $\Gamma\left(M, \stackrel{p}{\wedge}\left({ }^{\circ} T^{\prime \prime}\right)^{*}\right)$, we set

$$
\begin{aligned}
& L_{\xi}\left(u^{\prime} \otimes \xi\right)\left(X_{1}, X_{2}, \ldots, X_{p}\right) \\
& \quad=\left(\xi u^{\prime}\left(X_{1}, X_{2}, \ldots, X_{p}\right)\right. \\
& \left.\quad+\sum_{j}(-1)^{j} u^{\prime}\left(\left[\xi, X_{j}\right], X_{1}, \ldots, \check{X}_{j}, \ldots, X_{p}\right)\right) \otimes \xi .
\end{aligned}
$$

We consider $u^{\prime}$ as an element of $\Gamma\left(M, \stackrel{p}{\wedge}(C T M)^{*}\right)$ according to the splitting $C T M={ }^{\circ} T^{\prime \prime} \oplus^{\circ} \bar{T}^{\prime \prime} \oplus F$. Then the above definition of $L_{\xi}$ is rewritten as

$$
L_{\xi}\left(u^{\prime} \otimes \xi\right)\left(X_{1}, X_{2}, \ldots, X_{p}\right)=d u^{\prime}\left(\xi, X_{1}, X_{2}, \ldots, X_{p}\right) .
$$

Therefore $L_{\xi}$ is nothing but the Lie derivation in differential geometry. Then N. Tanaka proved that there exists a hermitian metric $g$ on $M$ such that

$$
L_{s} g=0
$$

in [3]. So we have the relation

$$
\sqrt{-1} \xi\left\langle u, u^{\prime}\right\rangle=\left\langle\sqrt{-1} L_{\xi} u, u^{\prime}\right\rangle-\left\langle u, \sqrt{-1} L_{\xi} u^{\prime}\right\rangle
$$

for all $u, u^{\prime}$ in $\Gamma\left(M, F \otimes \wedge\left({ }^{\circ} T^{\prime \prime}\right)^{*}\right)$, where by $\langle$,$\rangle we denote the$ inner product defined by the above metric $g$.

(2.7) We shall define an $L^{2}$-norm on $\Gamma\left(M, F \otimes \wedge\left({ }^{\circ} T^{\prime \prime}\right)^{*}\right)$ by the above inner product. Then $\sqrt{-1} L_{\xi}$ is a self adjoint operator by Proposition 3.1 in [3]. Moreover we have the following Proposition.

Proposition 2.7.1. If we put

$$
\square_{F}=\bar{\partial}_{F}^{(p)} \cdot \bar{\partial}_{F}^{*(p)}+\bar{\partial}_{F}^{*(p+1)} \cdot \bar{\partial}_{F}^{(p+1)},
$$


then we have

$$
\sqrt{-1} L_{\xi} \cdot \square_{F}=\square_{F} \cdot \sqrt{-1} L_{\xi},
$$

where $\bar{\partial}_{F}^{*(p)}$ denotes the $L^{2}$-adjoint operator of $\bar{\partial}_{F}^{(p)}$ and $p$ denotes a nonnegative integer.

Proof. Since $L_{\xi}$ is a Lie derivation, we have

$$
L_{\xi} \cdot \bar{\partial}_{F}^{(p)}=\bar{\partial}_{F}^{(p)} \cdot L_{\xi}
$$

Therefore it suffices to prove that

$$
L_{\xi} \cdot \bar{\partial}_{F}^{*(p)}=\bar{\partial}_{F}^{*(p)} \cdot L_{\xi}=\left(\varphi, \bar{\partial}_{F}^{(p)} \sqrt{-1} L_{\xi} \psi\right)=\left(\sqrt{-1} L_{\xi} \bar{\partial}_{F}^{*(p)} \varphi, \phi\right)
$$

In fact we have

$$
\left(\bar{\partial}_{F}^{*(p)} \sqrt{-1} L_{\xi} \varphi, \phi\right)=\left(\sqrt{-1} L_{\xi} \varphi, \bar{\partial}_{F}^{(p)} \phi\right)=\left(\varphi, \sqrt{-1} L_{\xi} \bar{\partial}_{F}^{(p)} \phi\right)
$$

for all $\varphi$ in $\Gamma\left(M, F \otimes \wedge^{p}\left({ }^{0} T^{\prime \prime}\right)^{*}\right)$ and $\psi$ in $\Gamma\left(M, F \otimes \bigwedge^{p-1}\left({ }^{0} T^{\prime \prime}\right)^{*}\right)$. This proves the proposition.

Q. E. D.

(2. 8) We speak of the harmonic theory on $\Gamma\left(M, F \otimes \wedge^{p}\left({ }^{0} T^{\prime \prime}\right)^{*}\right)$ by J. J. Kohn. In particular there exist the Neumann operator $N_{F}$ and the harmonic operator $\mathrm{H}_{F}$ with the relation $I=H_{F}+\square_{F} N_{F}$. From Proposition 2.7.1 it follows that $L_{\varepsilon}$ preserves the space of harmonic forms, and the following Corollary 2.8.1 holds.

Corollary 2. 8. 1. The following relation holds.

$$
N_{F} \cdot L_{\xi}=L_{\xi} \cdot N_{F} \text {. }
$$

\section{§3. A Priori Estimates for $D_{p}$}

We shall prove some a priori estimate in order to construct the versal family. The proofs of the estimates are very complicated exercises in integration by parts. To write down equations rather shortly, we shall state some notations.

For any $\varepsilon>0$ there exists $K>0$ such that for all positive numbers $a$ and $b, a b \leq \varepsilon a^{2}+K b^{2}$. We shall write this relation as 


$$
a b \leq\left(s . c_{.}\right) a^{2}+\left(l_{.} c_{.}\right) b^{2},
$$

where (l.c.) stands for "large constant" and (s.c.) stands for "small constant" with the understanding that $\left(s . c_{\text {. }}\right.$ ) may be chosen as small as it is necessary if we take (l. c.) sufficiently large. If $A$ and $B$ are real-valued functions on $a$ set $S$, we use the notation $A \leqslant B$ to mean that for some $c>0, A(\sigma) \leq c B(\sigma)$ for all $\sigma$ in $S$. If $A$ and $B$ also depend on other parameters, we shall say $A \lesssim B$ uniformly for $\sigma$ in $S$ to indicate that the constant $c$ is independent of $\sigma$, although not of the other parameters. Further, we write $A \sim B$ to mean that $A \leq B$ and $B \lesssim A$.

As usual let $C_{0}^{\infty}\left(R^{2 n-1}\right)$ denote the space of $C^{\infty}$ functions with compact supports on a euclidean space $R^{2 n-1}$. For each non-negative integer $m$, we define the Sobolev norms \|\|$_{(m)}$ on $C_{0}^{\infty}\left(R^{2 n-1}\right)$ by

$$
\|f\|_{(m)}^{2}=\sum_{|l| \leq m} \int_{R^{2 n-1}}\left|\partial^{l} / \partial x^{l} f(x)\right|^{2} d x
$$

where by $l$ we denote a multi index $\left(i_{1}, i_{2}, \ldots, i_{l}\right), 1 \leq i_{1} \leq i_{2} \leq \ldots \leq$ $i_{l} \leq 2 n-1$ and by $\partial^{l} / \partial x^{l} f$ we denote

$$
\partial^{l} / \partial x_{i_{1}} \partial x_{i_{2}} \ldots \partial x_{i_{l}} f
$$

where by $\left\{x_{i}\right\}_{1 \leq i \leq 2 n-1}$ we denote a real coordinate system of $R^{2 n-1}$.

Let $\left\{U_{k}, h_{k}\right\}_{k \in K}$ be an atlas of $M$ such that $K$ is a finite set and such that each $U_{k}$ is homeomorphic to $R^{2 n-1}$. (We are assuming that $M$ is compact s. p. c.) Let $\left\{\rho_{k}\right\}$ be a partition of unity subordinate to the atlas. For each $k$, take a moving frame $\left(e_{1}^{k}, e_{2}^{k}, \ldots, e_{n-1}^{k}\right)$ of $\left.{ }^{0} T^{\prime \prime}\right|_{U_{k}}$ and a moving frame $\left(\bar{e}_{1}^{k}, \bar{e}_{2}^{k}, \ldots, \bar{e}_{n-1}^{k}\right)$ of $\left.{ }^{0} \bar{T}^{\prime \prime}\right|_{U_{k}}$ such that $\left[\mathrm{e}_{i}^{k}, \bar{e}_{j}^{k}\right]_{F}=\sqrt{-1} \delta_{i, j} \xi i, j=1,2, \ldots, n-1$. By $J^{q}$ we denote the set of all ordered sets $\left(i_{1}, i_{2}, \ldots, i_{q}\right)$ of integers with $1 \leq i_{1}<i_{2}<\ldots<i_{q} \leq n-1$. For any $\varphi \in \Gamma\left(M,{ }^{0} \bar{T}^{\prime \prime} \otimes \bigwedge^{q}\left({ }^{0} T^{\prime \prime}\right)^{*}\right), I \in J^{q}$ and $l(1 \leq l \leq n-1)$, define $C^{\infty}$ functions $\underline{\varphi_{l, I}^{k}}$ on $U_{k}$ by

$$
\left(e_{i_{1}}^{k}, e_{i_{2}}^{k}, \ldots, e_{i_{q}}^{k}\right)=\sum_{l} \varphi_{l_{1} I}^{k} \bar{e}_{l}^{k},
$$

where $I=\left(i_{1}, i_{2}, \ldots, i_{q}\right)$. Using these functions, we define the Sobolev $m$-norm \|\|$_{(m)}$ in $\Gamma\left(M,{ }^{0} \bar{T}^{\prime \prime} \otimes \wedge^{q}\left({ }^{0} T^{\prime \prime}\right)^{*}\right)$ by

$$
\|\varphi\|_{(m)}^{2}=\sum_{l, I, k}\left\|\left(\rho_{k} \cdot \varphi_{l, I}^{k}\right) \circ h_{k}^{-1}\right\|_{(m)}^{2} .
$$


Moreover we define norms \|\|$_{(m)}^{\prime}$ and \|\|$_{(m)}^{\prime \prime}$ on $\Gamma\left(M,{ }^{\circ} \bar{T}^{\prime \prime} \otimes \wedge\left({ }^{0} T^{\prime \prime}\right)^{*}\right)$ by

$$
\begin{aligned}
\|\varphi\|_{(m)}^{2} & =\sum_{i, j, I, k}\left\|\rho_{k} e_{i}^{k}\left(\varphi_{j, I}^{k}\right) \circ h_{k}^{-1}\right\|_{(m)}^{2} \\
& +\sum_{i, j, I, k}\left\|_{k}^{1} \rho_{k} \bar{e}_{i}^{k}\left(\varphi_{j, I}^{k}\right) \circ h_{k}^{-1}\right\|_{(m)}^{2}+\|\varphi\|_{(m)}^{2}
\end{aligned}
$$

and

$$
\begin{aligned}
\|\varphi\|_{(m)}^{\prime \prime 2} & =\sum_{i, j, l, I, k}\left\|\rho_{k} e_{i}^{k} e_{j}^{k}\left(\varphi_{l, I}^{k}\right) \circ h_{k}^{-1}\right\|_{(m)}^{2} \\
& +\sum_{i, j, l, I, k}\left\|\rho_{k} \bar{e}_{i}^{k} e_{j}^{k}\left(\varphi_{l, I}^{k}\right) \circ h_{k}^{-1}\right\|_{(m)}^{2} \\
& +\sum_{i, j, l, I, k}\left\|\rho_{k} e_{i}^{k} \bar{e}_{j}^{k}\left(\varphi_{l, I}^{k}\right) \circ h_{k}^{-1}\right\|_{(m)}^{2} \\
& +\sum_{i, j, l, I, k}\left\|\rho_{k} \bar{e}_{i}^{k} \bar{e}_{j}^{k}\left(\varphi_{l, I}^{k}\right) \circ h_{k}^{-1}\right\|_{(m)}^{2}+\|\varphi\|_{(m)}^{2} .
\end{aligned}
$$

(From now on, we omit $\rho_{k}$ and $h_{k}^{-1}$ for brevity.)

J. J. Kohn [3] has shown that the methods of harmonic integrals work on an abstract s. p. c. manifold. In this section we assume that $(M, \xi)$ is a normal s. p. c. manifold of $\operatorname{dim}_{R} M=2 n-1 \geq 7$. Then we have the following Proposition 3.1 by Kohn's arguments.

Proposition 3.1. The following estimate

$$
\left\|D_{j-1}^{*} \varphi\right\|_{(0)}^{2}+\left\|D_{i} \varphi\right\|_{(0)}^{2}+\|\varphi\|_{(0)}^{2} \gtrsim\|\varphi\|_{(0)}^{2}
$$

holds uniformly for all $\varphi \in \Gamma\left(M,{ }^{\circ} \bar{T}^{\prime \prime} \otimes \wedge^{i}\left({ }^{0} T^{\prime \prime}\right)^{*}\right)$, where $D_{i}^{*}$ is an adjoint operator of $D_{i}$ with the above Sobolev 0 -norm, $i=1$ or 2 and we put $D_{0}^{*}=D^{*}$.

Proof. We only deal with the case $i=1$. The case $i=2$ can be shown analogously. For $\varphi$ in $\Gamma\left(M,{ }^{\circ} \bar{T}^{\prime \prime} \otimes\left({ }^{0} T^{\prime \prime}\right)^{*}\right)$, we have the relations

$$
\begin{aligned}
\left(D_{1} \varphi\right)\left(e_{i}^{k}, e_{j}^{k}\right) & =\left[e_{i}^{k}, \varphi\left(e_{j}^{k}\right)\right]_{0_{T^{\prime \prime}}}+\left[\varphi\left(e_{i}^{k}\right), e_{j}^{k}\right]_{0_{T^{\prime \prime}}}-\varphi\left(\left[e_{i}^{k}, e_{j}^{k}\right]\right) \\
& =\sum_{l}\left(e_{i}^{k} \varphi_{l, j}^{k}-e_{j}^{k} \varphi_{l, i}^{k}+\sum_{m, n} s_{i, j, l, m, n}^{k} \varphi_{m, n}^{k}\right) \bar{e}_{l}^{k},
\end{aligned}
$$

where $s_{i, j, l, m, n}^{k}$ denote $C^{\infty}$ functions on $U_{k}$, and

$$
\left(D^{*} \varphi\right)^{k}=\sum_{l}\left(-\sum_{i} \bar{e}_{i}^{k} \varphi_{l, i}^{k}+\sum_{m, n} t_{l, m, n}^{k} \varphi_{m, n}^{k}\right) \bar{e}_{l}^{k},
$$

where $t_{l, m, n}^{k}$ denote $C^{\infty}$ functions on $U_{k}$ by a simple computation using integration by parts. From (3.1) and (3.2) combined with the rela- 
tion $a b \leq(s . c.) a^{2}+($ l. c. $) b^{2}$, we have the following.

$$
\begin{aligned}
& \left\|_{1} D_{0}^{*} \varphi\right\|_{(0)}^{2}+\left\|D_{1} \varphi\right\|_{(0)}^{2}+\|\varphi\|_{(0)}^{2} \\
& \geq \sum_{l}\left\{\sum_{i<j, k}\left\|\left(e_{i}^{k} \varphi_{l, j}^{k}-e_{j}^{k} \varphi_{l, i}^{k}\right)\right\|_{(0)}^{2}\right. \\
& \left.+\left\|\left(-\sum_{i} \bar{e}_{i}^{k} \varphi_{l, i}^{k}\right)\right\|_{(0)}^{2}\right\} .
\end{aligned}
$$

We shall recall Kohn's argument. The right hand side of (3.3) becomes

$$
\begin{aligned}
\sum_{l}\left\{\sum _ { i < j , k } \left(\left\|e_{i}^{k} \varphi_{l, j}^{k}\right\|_{(0)}^{2}\right.\right. & \left.+\left\|e_{j}^{k} \varphi_{l, j}^{k}\right\|_{(0)}^{2}-2 \operatorname{Re}\left\langle e_{i}^{k} \varphi_{l, j}^{k}, e_{j}^{k} \varphi_{l, j}^{k}\right\rangle\right) \\
& \left.+\sum_{i}\left\|\bar{e}_{i}^{k} \varphi_{l, i}^{k}\right\|^{2}+\sum_{i<j, k} 2 \operatorname{Re}\left\langle\bar{e}_{j}^{k} \varphi_{l, j}^{k}, \quad \bar{e}_{i}^{k} \varphi_{l, i}^{k}\right\rangle\right\}
\end{aligned}
$$

While from the relation $\left[e_{i}^{k}, \bar{e}_{j}^{k}\right]_{F}=\sqrt{-1} \delta_{i, j} \xi$,

$$
\left\langle e_{i}^{k} \varphi_{l, j}^{k}, e_{j}^{k} \varphi_{l, i}^{k}\right\rangle=\left\langle\bar{e}_{i}^{k} \varphi_{l, j}^{k}, \bar{e}_{i}^{k} \varphi_{l, i}^{k}\right\rangle+\mathcal{O}\left(\|\varphi\|_{(0)}^{\prime}\|\varphi\|_{(0)}\right)
$$

holds integrating by parts, where $i \neq j$. From (3.3), (3.4) and (3.5), we have

$$
\begin{aligned}
\left\|D_{0}^{*} \varphi\right\|_{(0)}^{2} & +\left\|D_{1} \varphi\right\|_{(0)}^{2}+\left(l_{.} c_{.}\right)\|\varphi\|_{(0)}^{2} \\
& \geqq \sum_{l}\left\{\sum_{j}\left(\sum_{i \neq j}\left\|e_{i}^{k} \varphi_{l, j}^{k}\right\|_{(0)}^{2}+\left\|\overline{\boldsymbol{e}}_{j}^{k} \varphi_{l, j}^{k}\right\|_{(0)}^{2}\right)\right\} .
\end{aligned}
$$

Furthermore from the relation $\left[e_{j}^{k}, \bar{e}_{j}^{k}\right]_{F}=\sqrt{-1} \delta_{i, j} \xi$,

$$
\begin{aligned}
\left(e_{i}^{k} \varphi_{l, j}^{k}, e_{i}^{k} \varphi_{l, j}^{k}\right\rangle & =\left\langle\bar{e}_{i}^{k} \varphi_{l, j}^{k}, \bar{e}_{i}^{k} \varphi_{l, j}^{k}\right\rangle+\left\langle\sqrt{-1} \xi \varphi_{l, j}^{k}, \varphi_{l, j}^{k}\right\rangle \\
& +\mathcal{O}\left(\|\varphi\|_{(0)}^{\prime}\|\varphi\|_{(0)}\right)
\end{aligned}
$$

and

$$
\begin{aligned}
\left\langle\bar{e}_{j}^{k} \varphi_{l, j}^{k}, \bar{e}_{j}^{k} \varphi_{l, j}^{k}\right\rangle & =\left\langle e_{j}^{k} \varphi_{l, j}^{k}, e_{j}^{k} \varphi_{l, j}^{k}\right\rangle-\left\langle\sqrt{-1} \xi \varphi_{l, j}^{k}, \varphi_{l, j}^{k}\right\rangle \\
& +\mathcal{O}\left(\|\varphi\|_{(0)}^{\prime}\|\varphi\|_{(0)}\right) .
\end{aligned}
$$

From (3.6), (3.7) and (3.8), we have our Propositon. (For the details see Proposition 5.1 in J. J. Kohn [3].)

In this section we shall give more detailed estimates to construct the versal family.

\section{Proposition 3. 2. The following estimate}

$$
\left\|D_{i-1}^{*} \psi\right\|_{(m)}^{2}+\left\|D_{i} \psi\right\|_{(m)}^{2}+\|\psi\|_{(m)}^{2} \gtrsim\|\phi\|_{(m)}^{2}
$$

holds uniformly for all $\phi$ in $\Gamma\left(M,{ }^{0} \bar{T}^{\prime \prime} \otimes \wedge^{i}\left({ }^{0} T^{\prime \prime}\right)^{*}\right)$, where $m$ is a nonnegative integer and $i=1$ or 2 . 
Proof. It suffices to prove the inequality for forms $\phi$ supported in a patch $U_{k}$. Let $\left\{x_{i}\right\}_{1 \leq i \leq 2 n-1}$ be a real coordinate system of $U_{k}$. We define a differential operator $P_{L}$ on $\Gamma_{0}\left(U_{k},{ }^{0} \bar{T}^{\prime \prime} \otimes \wedge^{i}\left({ }^{0} T^{\prime \prime}\right)^{*}\right)$ to itself as follows : For $\phi$ in $\Gamma_{0}\left(U_{k},{ }^{0} \bar{T}^{\prime \prime} \otimes \wedge^{i}\left({ }^{0} T^{\prime \prime}\right)^{*}\right)$, we put

$$
\left(P_{L} \psi\right)_{l, I}^{k}=\partial / \partial x^{L} \phi_{l, I}^{k},
$$

where $I$ denotes a multi index $\left(i_{1}, i_{2}, \ldots, i_{l}\right)$. Further we put $s=|I|=\sum_{k=1}^{l} i_{k}$ of course

$$
\|\psi\|_{(s)}^{2} \sim \sum_{l, I, L}\left\|\left(P_{L} \psi\right)_{l}\right\|_{(0)}^{2} \quad|L| \leq s .
$$

We only deal with the case $i=1$. The case $i=2$ can be shown analogously. From Proposition 3.1, we have the estimate:

$$
\left\|D_{0}^{*} P_{L} \psi_{\|_{(0)}}^{2}+\right\| D_{1} P_{L} \psi\left\|_{(0)}^{2}+\right\| P_{L} \psi\left\|_{(0)}^{2} \gtrsim\right\| P_{L} \psi \|_{(0)}^{2}
$$

uniformly for all $\phi$ in $\Gamma_{0}\left(U_{k},{ }^{0} \bar{T}^{\prime \prime} \otimes\left({ }^{0} T^{\prime \prime}\right)^{*}\right)$. From the relation $D_{0}^{*} P_{L}=$ $P_{L} D_{0}^{*}+\left[D_{0}^{*}, P_{L}\right]$, we have that

$$
\left\|P_{L} D_{0}^{*} \psi\right\|_{(0)}^{2}+\left(l_{.} c_{.}\right)\|\psi\|_{(s)}^{2} \geq\left\|D_{0}^{*} P_{L} \psi\right\|_{(0)}^{2}, \quad|L|=s,
$$

uniformly for all $\phi$ in $\Gamma_{0}\left(U_{k},{ }^{0} \bar{T}^{\prime \prime} \otimes\left({ }^{0} T^{\prime \prime}\right)^{*}\right)$ by using the Schwarz inequality. Similarly we have that

$$
\left\|P_{L} D_{1} \psi\right\|_{(0)}^{2}+\left(l_{.} c_{.}\right)\|\psi\|_{(s)}^{2} \gtrsim\left\|D_{1} P_{L} \psi\right\|_{(0)}^{2}, \quad|L|=s,
$$

uniformly for all $\phi$ in $\Gamma_{0}\left(U_{k},{ }^{0} \bar{T}^{\prime \prime} \otimes\left({ }^{0} T^{\prime \prime}\right)^{*}\right)$. From (3.9), (3.10) and (3. 11), we have

$$
\left\|P_{L} D_{0}^{*} \psi\right\|_{i(0)}^{2}+\left\|P_{L} D_{1} \psi\right\|_{(0)}^{2}+\left(l_{.} c_{0}\right)\|\psi\|_{(s)}^{2} \geq\left\|P_{L} \psi\right\|_{(0)}^{2},
$$

uniformly for all $\psi$ in $\Gamma_{0}\left(U_{k},{ }^{0} \bar{T}^{\prime \prime} \otimes\left({ }^{0} \bar{T}^{\prime \prime}\right)^{*}\right)$. Therefore we have that

$$
\left\|D_{0}^{*} \psi\right\|_{(m)}^{2}+\left\|D_{1} \psi\right\|_{(m)}^{2}+\left(l_{0} c .\right)\|\psi\|_{(m)}^{2} \gtrsim \sum_{|L| \leq m} \| P_{L} \psi_{\|_{(0)}^{\prime}}^{2},
$$

uniformly for all $\phi$ in $\Gamma_{0}\left(U_{k},{ }^{\circ} \bar{T}^{\prime \prime} \otimes\left({ }^{0} T^{\prime \prime}\right)^{*}\right)$. And from the relations $e_{i}^{k} \cdot P_{L}=P_{L} \cdot e_{i}^{k}+\left[e_{i}^{k}, P_{L}\right]$ and $\bar{e}_{i}^{k} \cdot P_{L}=P_{L} \cdot \bar{e}_{i}^{k}+\left[\bar{e}_{i}^{k}, P_{L}\right]$, we have

$$
\sum_{|L| \leq m}\left\|P_{L} \psi\right\|_{(0)}^{2}+(l . c .)\left\|\left.\phi\right|_{(m)} ^{2} \geq\right\| \psi \|_{(m)}^{2},
$$

uniformly for all $\phi$ in $\Gamma_{0}\left(U_{k},{ }^{\circ} \bar{T}^{\prime \prime} \otimes\left({ }^{0} T^{\prime \prime}\right)^{*}\right)$. From (3.13) and (3.14), we have our Proposition.

Q. E. D.

From Proposition 3.2, we shall prove the key estimates. 
Proposition 3. 3. For any non-negative integer $m$, the following estimate

$$
\|\psi\|_{(m)}^{\prime_{2}} \lesssim\|\square \phi\|_{(m)}^{2}+\|\psi\|_{(m)}^{2}
$$

holds uniformly for all $\phi$ in $\Gamma\left(M,{ }^{\circ} \bar{T}^{\prime \prime} \otimes\left({ }^{0} T^{\prime \prime}\right)^{*}\right)$, where $\square$ denotes $D_{1}^{*} \cdot D_{1}+D_{0} \cdot D_{0}^{*}$.

In order to prove this proposition, we shall prove some lemmas.

Lemma 3.4. For any non-negative integer $m$, the following estimate

$$
\|\psi\|_{(m+1)}^{2} \leqq\|\square \psi\|_{(m)}^{2}+\|\psi\|_{(m)}^{2}
$$

holds uniformly for all $\phi$ in $\Gamma\left(M,{ }^{\circ} \bar{T}^{\prime \prime} \otimes\left({ }^{0} T^{\prime \prime}\right)^{*}\right)$.

For the proof see Theorem 2.4 in [2]. In [2] the estimate of the above type is proved for scalar valued forms. The proof for ${ }^{\circ} \bar{T}^{\prime \prime}$-valued forms is similar.

Lemma 3. 5. Under the above situation, the following estimate holds uniformly for all $\phi$ in $\Gamma_{0}\left(U_{k},{ }^{\circ} \bar{T}^{\prime \prime} \otimes\left({ }^{0} T^{\prime \prime}\right)^{*}\right)$ :

$$
\|\phi\|_{i(m)}^{2}+\left(l_{.} c_{.}\right)\|\phi\|_{(m+1)}^{2} \sim \sum_{|L| \leqq m}\left\|P_{L} \psi\right\|_{(0)}^{2}+\left(l_{.} c_{.}\right)\|\phi\|_{(m+1)}^{2}
$$

Proof. From the definition, we have

$$
\|\phi\|_{(m)}^{2} \sim \sum_{|L| \leq m} \sum_{l, j}\left\|\left(P_{L} \psi\right)_{l, j}^{k}\right\|_{(0)}^{2} .
$$

From the relation $P_{L} \square=\square P_{L}+\left[P_{L}, \square\right]$ and that $\left[P_{L}, \square\right]$ is a differential operator of order $|L|+1$, we have

$$
\begin{aligned}
& \sum_{|L|=m} \sum_{l, j}\left\|\left(P_{L} \square \psi\right)_{l, j}^{k}\right\|_{(0)}^{2}+\left(l_{.} c_{.}\right)\|\phi\|_{|L|+1} \\
& \quad \sim \sum_{|L|=m} \sum_{l, j}\left\|\left(\square P_{L}\right) \psi_{l, j}^{k}\right\|_{(0)}^{2}+\left(l_{.} c_{0}\right)\|\phi\|_{|L|+1}
\end{aligned}
$$

by using Schwarz inequality and the relation $a b \leq\left(s_{.} c_{0}\right) a^{2}+\left(l_{0} c_{.}\right) b^{2}$. Therefore we have Lemma 3.5. Q. E. D.

Using Lemma 3.4 and Lemma 3.5, we shall prove Proposition 3.3. It suffices to prove the inequality for forms $\phi$ supported in a 
patch $U_{k}$. By Lemma 3.4 and Lemma 3.5, if the following inequality (3.15) holds, our Proposition 3.3 is proven.

$$
\|\psi\|_{(0)}^{\prime_{2}} \lesssim\|\square \phi\|_{(0)}^{2}+\|\psi\|_{(1)}^{2},
$$

uniformly for $\phi \in \Gamma_{0}\left(U_{k},{ }^{\circ} \bar{T}^{\prime \prime} \otimes\left({ }^{0} T^{\prime \prime}\right) *\right)$.

In fact setting $\varphi=P_{L} \psi$ in this inequality, we have the following inequality with $\psi \in \Gamma_{0}\left(U_{k},{ }^{0} \bar{T}^{\prime \prime} \otimes\left({ }^{0} T^{\prime \prime}\right)^{*}\right)$

$$
\left\|P_{L} \psi\right\|_{(0)}^{\prime 2} \leqq\left\|\square P_{L} \psi\right\|_{(0)}^{2}+\left\|P_{L} \psi\right\|_{(1)}^{2} \text {. }
$$

And so

$$
\left\|P_{L} \psi\right\|_{(0)}^{\prime \prime 2} \lesssim\left\|\square P_{L} \psi\right\|_{(0)}^{2}+\|\psi\|_{(|L|+1)}^{2} .
$$

On the other hand, the following relation (3.18) holds for all $\phi$ in $\Gamma_{0}\left(U_{k},{ }^{0} \bar{T}^{\prime \prime} \otimes\left({ }^{0} T^{\prime \prime}\right)^{*}\right)$.

$$
\begin{gathered}
\sum_{|L| \leq m} \sum_{l, r}\left\|\left(e_{i}^{k} e_{j}^{k} P_{L} \psi\right)_{l, r}^{k}\right\|^{2}+\left(l_{.} c_{.}\right)\|\psi\|_{(m+1)}^{2} \\
\sim \sum_{|L| \leq m} \sum_{l, r}\left\|\left(P_{L} e_{i}^{k} e_{j}^{k} \psi\right)_{l, r}^{k}\right\|^{2}+\left(l_{.} c_{.}\right)\|\psi\|_{(m+1)}^{2} .
\end{gathered}
$$

In fact from the relation $e_{i}^{k} \cdot e_{j}^{k} P_{L}=P_{L} e_{i}^{k} e_{j}^{k}+\left[e_{i}^{k} e_{j}^{k}, P_{L}\right]$ and Schwarz inequality, (3.18) follows. Similarly we have

$$
\begin{aligned}
& \sum_{|L| \leq m} \sum_{l, r}\left\|\left(e_{i}^{k} \bar{e}_{j}^{k} P_{L} \psi\right)_{l, r}^{k}\right\|_{(0)}^{2}+\left(l_{0} c_{0}\right) \| \phi_{\|_{(m+1)}}^{2} \\
& \sim \sum_{|L| \leq m} \sum_{l, r}\left\|\left(P_{L} e_{i}^{k} \bar{e}_{j}^{k} \psi\right)_{l, r}^{k}\right\|_{(0)}^{2}+\left(l_{.} c_{.}\right)\|\phi\|_{(m+1)}^{2}, \\
& \begin{array}{r}
\sum_{|L| \leq m} \sum_{l, r}\left\|\left(\bar{e}_{i}^{k} e_{j}^{k} P_{L} \psi\right)_{l, r}^{k}\right\|_{(0)}^{2}+\left(l_{.} c_{.}\right)\|\psi\|_{(m+1)}^{2} \\
\sim \sum_{|L| \leq m} \sum_{l, r}\left\|\left(P_{L} \bar{e}_{i}^{k} e_{j}^{k} \psi\right)_{l, r}^{k}\right\|_{(0)}^{2}+\left(l_{.} c_{.}\right)\|\psi\|_{(m+1)}^{2}
\end{array}
\end{aligned}
$$

and

$$
\begin{aligned}
& \sum_{|L| \leq m} \sum_{l, r}\left\|\left(\bar{e}_{i}^{k} e_{j}^{k} P_{L} \psi\right)_{l, r}^{k}\right\|_{(0)}^{2}+\left(l_{.} c_{.}\right)\|\psi\|_{(m+1)}^{2} \\
\sim & \sum_{|L| \leq m} \sum_{l, r}\left\|\left(P_{L} \bar{e}_{i}^{k} \bar{e}_{j}^{k} \psi\right)_{l, r}^{k}\right\|_{(0)}^{2}+\left(l_{.} c_{0}\right)\|\psi\|_{(m+1)}^{2}
\end{aligned}
$$

for all $\psi$ in $\Gamma_{0}\left(U_{k},{ }^{0} \bar{T}^{\prime \prime} \otimes\left({ }^{0} T^{\prime \prime}\right)^{*}\right)$. From (3.18), (3.19), (3.20) and (3. 21), we have

$$
\begin{aligned}
& \sum_{|L| \leq m}\left\|P_{L} \psi\right\|_{(0)}^{\prime \prime 2}+(l . c .)\|\phi\|_{(m+1)}^{2} \\
\sim & \|\phi\|_{(m)}^{\prime \prime 2}+(l . c .)\|\psi\|_{(m+1)}^{2} .
\end{aligned}
$$

From these relations and Lemmas 3.4 and 3.5, we have Proposition 
3. 3.

We shall prove the estimate (3.15). From Proposition 3.1 applied to $D_{1}$ and taking into account the relation $D_{2} D_{1}=0$ as follows from the normality of $\left(M,{ }^{\circ} T^{\prime \prime}\right)$, we have the relation:

$$
\left\|D_{1} \varphi\right\|_{(0)}^{2} \lesssim\left\|D_{1}^{*} D_{1} \varphi\right\|_{(0)}^{2}+\left\|D_{1} \varphi\right\|_{(0)}^{2},
$$

for all $\varphi \in \Gamma\left(M,{ }^{\circ} \bar{T}^{\prime \prime} \otimes\left({ }^{0} T^{\prime \prime}\right)^{*}\right)$.

We put

$$
\varphi\left(e_{i}^{k}\right)=\sum_{l} \varphi_{l, i}^{k} \bar{e}_{l}^{k}, \quad \varphi \in \Gamma\left(M,{ }^{0} \bar{T}^{\prime \prime} \otimes\left({ }^{0} T^{\prime \prime}\right)^{*}\right) .
$$

Then

$$
\left(D_{1} \varphi\right)_{m,(n, l)}^{k}=e_{n}^{k} \varphi_{m, l}^{k}-e_{l}^{k} \varphi_{m, n}^{k}+\mathcal{O}\left(\|\varphi\|_{(0)}\right) .
$$

From this and (3.23), we have

$$
\begin{aligned}
& \sum_{m}\left\{\sum_{i, n<l}\left\|\bar{e}_{i}^{k}\left(e_{n}^{k} \varphi_{m, l}^{k}-e_{l}^{k} \varphi_{m, n}^{k}\right)\right\|_{(0)}^{2}\right\} \\
& \leq\left\|D_{1}^{*} D_{1} \varphi\right\|_{(0)}^{2}+\left(l_{.} c_{.}\right)\|\varphi\|_{(1)}^{2}
\end{aligned}
$$

by Schwarz lemma and using the relation $a b \leq\left(s_{*} c_{*}\right) a^{2}+\left(l_{*} c_{.}\right) b^{2}$. On the other hand, since

$$
\left(D^{*} \varphi\right)_{m}^{k}=-\sum_{l} \bar{e}_{l}^{k} \varphi_{m, l}^{k}+\mathcal{O}\left(\|\varphi\|_{(0)}\right),
$$

we have

$$
\left(D D^{*} \varphi\right)_{m, i}^{k}=e_{i}^{k}\left(-\sum_{l} \bar{e}_{l}^{k} \varphi_{m, l}^{k}\right)+\mathcal{O}\left(\|\varphi\|_{(0)}^{\prime}\right)
$$

We shall compute $\left\|D D^{*} \varphi\right\|_{(0)}^{2}$ by this formula.

Lemma 3. 6.

$$
\begin{aligned}
& \left\|D D^{*} \varphi\right\|_{(0)}^{2}+\left(l_{.} c_{.}\right)\|\varphi\|_{(1)}^{2}+\left(s_{.} c_{.}\right)\|\varphi\|_{(0)}^{\prime_{2}^{2}} \\
& \sim \sum_{m}\left\{\sum_{i}\left\{\sum_{l}\left\|e_{i}^{k} \bar{e}_{l}^{k} \varphi_{m, l}^{k}\right\|_{(0)}^{2}+2 \operatorname{Re} \sum_{l<n}\left\langle e_{i}^{k} \bar{e}_{l}^{k} \varphi_{m, l}^{k}, e_{i}^{k} \bar{e}_{n}^{k} \varphi_{m, n}^{k}\right\rangle\right\}\right\} .
\end{aligned}
$$

Proof. From (3.25) we have Lemma 3.6 by using integration by parts.

Q. E. D.

And by a simple calculation we have:

\section{Lemma 3. 7.}




$$
\begin{aligned}
& \sum_{m}\left\{\sum_{i, n<l}\left\|\bar{e}_{i}^{k}\left(e_{n}^{k} \varphi_{m, l}^{k}-e_{l}^{k} \varphi_{m, n}^{k}\right)\right\|_{(0)}^{2}\right\} \\
= & \sum_{m}\left\{\sum_{i, n \leq l}\left\|\bar{e}_{i}^{k} e_{n}^{k} \varphi_{m, l}^{k}\right\|_{(0)}^{2}+\left\|\bar{e}_{i}^{k} e_{l}^{k} \varphi_{m, n}^{k}\right\|_{(0)}^{2}\right. \\
- & \left.2 \operatorname{Re}\left\langle\bar{e}_{i}^{k} e_{n}^{k} \varphi_{m, l}^{k}, \bar{e}_{i}^{k} e_{l}^{k} \varphi_{m, n}^{k}\right\rangle\right\} .
\end{aligned}
$$

We shall prove the following estimate.

Lemma 3. 8. The terms

$$
\begin{array}{r}
2 \operatorname{Re} \sum_{i, l>n}\left\langle e_{i}^{k} e_{l}^{k} \varphi_{m, l}^{k}, e_{i}^{k} \bar{e}_{n}^{k} \varphi_{m, n}^{k}\right\rangle \\
-2 \operatorname{Re} \sum_{i, l>n}\left\langle\bar{e}_{i}^{k} e_{n}^{k} \varphi_{m, l}^{k}, \bar{e}_{i}^{k} e_{l}^{k} \varphi_{m, n}^{k}\right\rangle
\end{array}
$$

can be estimated by $\left(s_{0} c_{.}\right)\|\varphi\|_{(0)}^{2_{2}}+\left(l_{.} c_{.}\right)\|\varphi\|_{(1)}^{2}$.

Proof. We only prove the estimate

$$
\begin{aligned}
& 2 \operatorname{Re} \sum_{i, l>n}\left\langle e_{i}^{k} \bar{e}_{l}^{k} \varphi_{m, l}^{k}, e_{i}^{k} \bar{e}_{n}^{k} \varphi_{m, n}^{k}\right\rangle \\
\leq & 2 \operatorname{Re} \sum_{i, l>n}\left\langle\bar{e}_{i}^{k} e_{n}^{k} \varphi_{m, l}^{k}, \bar{e}_{i}^{k} e_{l}^{k} \varphi_{m, n}^{k}\right\rangle+(s . c .)\|\varphi\|_{(0)}^{\prime \prime 2}+\left(l_{.} c_{.}\right)\|\varphi\|_{(1)}^{2}
\end{aligned}
$$

uniformly for all $\varphi \in \Gamma_{0}\left(U_{k},{ }^{\circ} \bar{T}^{\prime \prime} \otimes\left({ }^{\circ} T^{\prime \prime}\right)^{*}\right)$.

We count $\operatorname{Re}\left\langle e_{i}^{k} \bar{e}_{l}^{k} \varphi_{m, l}^{k}, e_{i}^{k} e_{n}^{k} \varphi_{m, n}^{k}\right\rangle$ by integration by parts.

$$
\begin{aligned}
& \operatorname{Re}\left\langle e_{i}^{k} \bar{e}_{l}^{k} \varphi_{m, l}^{k}, e_{i}^{k} \bar{e}_{n}^{k} \varphi_{m, n}^{k}\right\rangle \\
= & -\operatorname{Re}\left\langle\bar{e}_{i}^{k} e_{i}^{k} \bar{e}_{l}^{k} \varphi_{m, l}^{k}, \quad \bar{e}_{n}^{k} \varphi_{m, n}^{k}\right\rangle+\mathcal{O}\left(\|\varphi\|_{(0)}^{\prime \prime}\|\varphi\|_{(1)}\right) \\
= & -\operatorname{Re}\left\langle e_{i}^{k} \bar{e}_{i}^{k} \bar{e}_{l}^{k} \varphi_{m, l}^{k}, \quad \bar{e}_{n}^{k} \varphi_{m, n}^{k}\right\rangle \\
& -\left\langle\operatorname{Re}\left[\bar{e}_{i}^{k}, e_{i}^{k}\right] \bar{e}_{l}^{k} \varphi_{m, l}^{k}, \bar{e}_{n}^{k} \varphi_{m, n}^{k}\right\rangle+\mathcal{O}\left(\|\varphi\|_{(0)}^{\prime \prime}\left\|_{l} \mid \varphi\right\|_{(1)}\right) .
\end{aligned}
$$

The term $\operatorname{Re}\left\langle\left[\bar{e}_{i}^{k}, e_{i}^{k}\right] \bar{e}_{l}^{k} \varphi_{m, l}^{k}, e_{n}^{k} \varphi_{m, n}^{k}\right\rangle$ can be estimated as follows:

$$
\begin{aligned}
& \operatorname{Re}\left\langle\left[\bar{e}_{i}^{k}, e_{i}^{k}\right] \bar{e}_{l}^{k} \varphi_{m, l}^{k}, \bar{e}_{n}^{k} \varphi_{m, n}^{k}\right\rangle \\
= & \operatorname{Re}\left\langle\bar{e}_{l}^{k}\left[\bar{e}_{i}^{k}, e_{i}^{k}\right] \varphi_{m, l}^{k}, \bar{e}_{n}^{k} \varphi_{m, n}^{k}\right\rangle \\
& +\operatorname{Re}\left\langle\left[\left[\bar{e}_{i}^{k}, e_{i}^{k}\right], \bar{e}_{l}^{k}\right] \varphi_{m, l}^{k}, \bar{e}_{n}^{k} \varphi_{m, n}^{k}\right\rangle \\
= & -\operatorname{Re}\left\langle\left[\bar{e}_{i}^{k}, e_{i}^{k}\right] \varphi_{m, l}^{k}, e_{e}^{k} e_{n}^{k} \varphi_{m, n}^{k}\right\rangle+\mathcal{O}\left(\|\varphi\|_{(0)}^{\prime}\|\varphi\|_{(1)}\right) \\
\leq & \left(l_{.} c_{.}\right)\|\varphi\|_{(1)}^{2}+\left(s, c_{0}\right)\|\varphi\|_{(0)}^{2} .
\end{aligned}
$$

So we may neglect this term.

Therefore from (3.26) we have

$$
\begin{aligned}
& \operatorname{Re}\left\langle e_{i}^{k} \bar{e}_{l}^{k} \varphi_{m, l}^{k}, e_{i}^{k} e_{n}^{k} \varphi_{m, n}^{k}\right\rangle \\
& \leq-\operatorname{Re}\left\langle e_{i}^{k} \bar{e}_{i}^{k} \bar{e}_{l}^{k} \varphi_{m, l}^{k}, \bar{e}_{n}^{k} \varphi_{m, n}^{k}\right\rangle+(\text { l.c. })\left\|\varphi !_{(1)}^{2}+\left(s_{0} . c_{0}\right)\right\| \varphi \|_{(0)}^{\prime \prime 2} \\
& \leq \operatorname{Re}\left\langle\bar{e}_{i}^{k} \bar{e}_{l}^{k} \varphi_{m, l}^{k}, \bar{e}_{i}^{k} \bar{e}_{n}^{k} \varphi_{m, n}^{k}\right\rangle+\left(l^{k} c_{.}\right)\|\varphi\|_{(1)}^{2}+(s . c .)\|\varphi\|_{(0)}^{n_{2}} \text {. }
\end{aligned}
$$


Furthermore the term

$$
\operatorname{Re}\left\langle\bar{e}_{i}^{k} \bar{e}_{l}^{k} \varphi_{m, l}^{k}, \bar{e}_{i}^{k} \bar{e}_{n}^{k} \varphi_{m, n}^{k}\right\rangle
$$

can be estimated as follows :

$$
\begin{aligned}
& \operatorname{Re}\left\langle\bar{e}_{i}^{k} \bar{e}_{l}^{k} \varphi_{m, l}^{k}, \bar{e}_{i}^{k} \bar{e}_{n}^{k} \varphi_{m, n}^{k}\right\rangle \\
& \leq \operatorname{Re}\left\langle\bar{e}_{l}^{k} \bar{e}_{i}^{k} \varphi_{m, l}^{k}, \bar{e}_{i}^{k} \bar{e}_{n}^{k} \varphi_{m, n}^{k}\right\rangle+\operatorname{Re}\left\langle\left[\bar{e}_{i}^{k}, \bar{e}_{l}^{k}\right] \varphi_{m, l}^{k}, \bar{e}_{i}^{k} \bar{e}_{n}^{k} \varphi_{m, n}^{k}\right\rangle \\
& \leq \operatorname{Re}\left\langle\bar{e}_{l}^{k} \bar{e}_{i}^{k} \varphi_{n, l}^{k}, \bar{e}_{i}^{k} \bar{e}_{n}^{k} \varphi_{m, n}^{k}\right\rangle+\left(\text { s.c.) }\|\varphi\|_{(0)}^{\prime 2}+\left(l_{\text {. c. }}\right)\|\varphi\|_{(1)}^{2}\right. \\
& \leq \operatorname{Re}\left\langle\bar{e}_{l}^{k} \bar{e}_{i}^{k} \varphi_{m, l}^{k}, \bar{e}_{n}^{k} \bar{e}_{i}^{k} \varphi_{m, n}^{k}\right\rangle+\operatorname{Re}\left\langle\bar{e}_{l}^{k} \bar{e}_{i}^{k} \varphi_{m, l}^{k}\left[\bar{e}_{i}^{k}, \bar{e}_{n}^{k}\right] \varphi_{m, n}^{k}\right\rangle \\
& +(s . c .)\|\varphi\|_{(0)}^{\prime \prime 2}+\left(l_{0} c_{.}\right)\|\varphi\|_{(1)}^{2} \\
& \leq \operatorname{Re}\left\langle\bar{e}_{l}^{k} \bar{e}_{i}^{k} \varphi_{m, l}^{k}, \bar{e}_{n}^{k} \bar{e}_{i}^{k} \varphi_{m, n}^{k}\right\rangle+\left(s . c_{0}\right)\|\varphi\|_{(0)}^{\prime \prime 2}+\left(l_{0} c_{0}\right)\|\varphi\|_{(1)}^{2} \cdot
\end{aligned}
$$

The term $\operatorname{Re}\left\langle\bar{e}_{l}^{k} \bar{e}_{i}^{k} \varphi_{m, l}^{k}, \bar{e}_{n}^{k} \bar{e}_{i}^{k} \varphi_{m, n}^{k}\right\rangle$ is estimated as follows.

$$
\begin{aligned}
& \operatorname{Re}\left\langle\bar{e}_{l}^{k} \bar{e}_{i}^{k} \varphi_{m, l}^{k}, \bar{e}_{n}^{k} \bar{e}_{i}^{k} \varphi_{m, n}^{k}\right\rangle \\
& \leq-\operatorname{Re}\left\langle e_{n}^{k} \bar{e}_{l}^{k} \bar{e}_{i}^{k} \varphi_{m, l}^{k}, \bar{e}_{i}^{k} \varphi_{m, n}^{k}\right\rangle+\left(\text { s. c.) }\|\varphi\|_{(0)}^{\prime \prime 2}+(\text { l.c. })\|\varphi\|_{(1)}^{2}\right. \\
& \leq-\operatorname{Re}\left\langle\bar{e}_{l}^{k} e_{n}^{k} \bar{e}_{i}^{k} \varphi_{m, l}^{k}, \bar{e}_{i}^{k} \varphi_{m, n}^{k}\right\rangle+\operatorname{Re}\left\langle\left[\bar{e}_{l}^{k}, e_{n}^{k}\right] \bar{e}_{i}^{k} \varphi_{m, l}^{k}, \bar{e}_{i}^{k} \varphi_{m, n}^{k}\right\rangle \\
& +\left(s_{.} c_{0}\right)\|\varphi\|_{(0)}^{\prime \prime 2}+\left(l_{\text {. c. }}\right)\|\varphi\|_{(1)}^{2} \\
& \leq-\operatorname{Re}\left\langle\bar{e}_{l}^{k} e_{n}^{k} \bar{e}_{i}^{k} \varphi_{m, l}^{k}, \bar{e}_{i}^{k} \varphi_{m, n}^{k}\right\rangle+\left(s_{0} c_{0}\right)\|\varphi\|_{(0)}^{\prime \prime 2}+\left(l_{0} c_{0}\right)\|\varphi\|_{(1)}^{2} \\
& \leq \operatorname{Re}\left\langle e_{n}^{k} \bar{e}_{i}^{k} \varphi_{m, l}^{k}, e_{l}^{k} \bar{e}_{i}^{k} \varphi_{m, n}^{k}\right\rangle+\left(\text { s.c.) }\|\varphi\|_{(0)}^{\prime \prime 2}+\left(l_{\text {. c. }}\right)\|\varphi\|_{(1)}^{2}\right. \\
& \leq \operatorname{Re}\left\langle\bar{e}_{i}^{k} e_{n}^{k} \varphi_{m, l}^{k}, e_{l}^{k} \bar{e}_{i}^{k} \varphi_{m, n}^{k}\right\rangle+\operatorname{Re}\left\langle\left[e_{n}^{k}, \bar{e}_{i}^{k}\right] \varphi_{m, l}^{k}, e_{l}^{k} \bar{e}_{i}^{k} \varphi_{m, n}^{k}\right\rangle \\
& +(s . c .)\left\|_{1}^{1} \varphi\right\|_{(0)}^{\prime \prime 2}+\left(l_{0} c_{0}\right)\|\varphi\|_{(1)}^{2} \\
& \leq \operatorname{Re}\left\langle\bar{e}_{i}^{k} e_{n}^{k} \varphi_{m, l}^{k}, e_{l}^{k} \bar{e}_{i}^{k} \varphi_{m, n}^{k}\right\rangle+\left(s_{.} c_{\text {. }}\right)\|\varphi\|_{(0)}^{\prime \prime 2}+\left(l_{\text {. }} c_{0}\right)\|\varphi\|_{(1)}^{2} \\
& \leq \operatorname{Re}\left\langle\bar{e}_{i}^{k} e_{n}^{k} \varphi_{m, l}^{k}, \quad \bar{e}_{i}^{k} e_{l}^{k} \varphi_{m, n}^{k}\right\rangle+\operatorname{Re}\left\langle\bar{e}_{i}^{k} e_{n}^{k} \varphi_{m, l}^{k}, \quad\left[e_{l}^{k}, \bar{e}_{i}^{k}\right] \varphi_{m, n}^{k}\right\rangle \\
& +\left(s . c_{0}\right)\|\varphi\|_{(0)}^{\prime 2}+\left(l_{0} c_{0}\right)\|\varphi\|_{(1)}^{2} \\
& \leq \operatorname{Re}\left\langle\bar{e}_{i}^{k} e_{n}^{k} \varphi_{m, l}^{k}, \bar{e}_{i}^{k} e_{l}^{k} \varphi_{m, n}^{k}\right\rangle+\left(s_{\bullet} c_{.}\right)\left\|\varphi_{\|}^{\prime}\right\|_{(0)}^{\prime \prime 2}+\left(l_{\bullet} c_{.}\right)\|\varphi\|_{(1)}^{2} \text {. }
\end{aligned}
$$

So we have Lemma 3.8.

From Lemma 3.6, Lemma 3.7 and Lemma 3.8 we have the estimate (3.15). Therefore we have Proposition 3.3.

From Proposition 3.3 we have the following key estimate.

Proposition 3. 9. Under the situation in Proposition 3. 3, the following estimate holds uniformly $\mu \in \Gamma\left(M,{ }^{\circ} \bar{T}^{\prime \prime} \otimes\left({ }^{0} T^{\prime \prime}\right)^{*}\right)$.

$$
\|N \mu\|_{(m)}^{\prime \prime} \lesssim\|\mu\|_{(m)}
$$

where $N$ denotes the Neumann operator for $\square$ and $m$ denotes a non- 
negative integer.

Proof. The following estimate is proved in Proposition 3. 3.

$$
\|\varphi\|_{(m)}^{\prime 2} \leqq\|\square \varphi\|_{(m)}^{2}+\|\varphi\|_{(m)}^{2}
$$

for all $\varphi$ in $\Gamma\left(M,{ }^{0} T^{\prime \prime} \otimes\left({ }^{0} T^{\prime \prime}\right)^{*}\right)$. Setting $\varphi=N \mu$ in this estimate, we have

$$
\begin{aligned}
\|N \mu\|_{(m)}^{\prime \prime 2} & \leqq\|\square N \mu\|_{(m)}^{2}+\|N \mu\|_{(m)}^{2}, \\
& \leqq\|\mu-H \mu\|_{(m)}^{2}+\|N \mu\|_{(m)}^{2},
\end{aligned}
$$

where $H$ denotes the harmonic operator for $\square$. While

$$
\|N \mu\|_{(m)}^{2} \lesssim\|\mu\|_{(m)}^{2}
$$

and

$$
\|H \mu\|_{(m)}^{2} \leqq\|\mu\|_{(m)}^{2}
$$

holds uniformly for $\mu$ in $\Gamma\left(M,{ }^{\circ} \bar{T}^{\prime \prime} \otimes\left({ }^{0} T^{\prime \prime}\right)^{*}\right)$. So Proposition 3.9 is proven.

We shall take the Hermitian metric along the fibres of $T^{\prime}={ }^{0} \bar{T}^{\prime \prime} \oplus F$, and make the space of $C^{\infty}$-sections of $T^{\prime} \otimes \wedge^{i}\left({ }^{0} T^{\prime \prime}\right) *$ pre-Hilbert spaces. We take the Sobolev n-norm on there. We shall also introduce the adjoint operator $\bar{\partial}_{T^{\prime}}^{*}$ of $\bar{\partial}_{T^{\prime}}$, making use of this metric, and consider the sub-elliptic differential operator $\square_{T^{\prime}}=\bar{\partial}_{T^{\prime}}^{*(i)} \cdot \bar{\partial}_{T^{\prime}}^{(i)}+\bar{\partial}_{T^{\prime}}^{(i-1)} \cdot \bar{\partial}_{T^{\prime}}^{*(i-1)}$. By a similar argument we have the following Propositions.

Proposition 3.10. The estimate

$$
\|\mu\|_{(m)}^{\prime 2} \lesssim\left\|\square_{T^{\prime}} \mu\right\|_{(m)}^{2}+\|\mu\|_{(m)}^{2}
$$

holds uniformly for all $\mu$ in $\Gamma\left(M, T^{\prime} \otimes\left({ }^{0} T^{\prime \prime}\right)^{*}\right)$ and for all $m$ nonnegative integer.

Proposition 3.11. The estimate

$$
\left\|N_{T^{\prime}} \mu\right\|_{(m)}^{\prime \prime} \leqq\|\mu\|_{(m)}
$$

holds uniformly for all $\mu$ in $\Gamma\left(M, T^{\prime} \otimes\left({ }^{\circ} T^{\prime \prime}\right)^{*}\right)$ and for all $m$ nonnegative integer, where $N_{T^{\prime}}$ denotes the Neumann operator for $\square_{T^{\prime}}$. 
Proposition 3.12. The following estimate

$$
\left\|D_{1}^{*} R_{2}(\varphi)\right\|_{(m)} \lesssim\|\varphi\|_{(m)}^{\prime 2}
$$

holds uniformly for all $\varphi$ in $\Gamma\left(M,{ }^{\circ} \bar{T}^{\prime \prime} \otimes\left({ }^{0} T^{\prime \prime}\right)^{*}\right)$.

Proof. In fact from the relation

$$
R_{2}(\varphi)(X, Y)=[\varphi(X), \varphi(Y)]_{T^{\prime}}-\varphi\left([\varphi(X), Y]_{0_{T^{\prime \prime}}}+[X, \varphi(Y)]_{{ }_{T^{\prime \prime}}}\right)
$$

and

$$
\varphi \in \Gamma\left(M,{ }^{0} \bar{T}^{\prime \prime} \otimes\left({ }^{0} T^{\prime \prime}\right)^{*}\right)
$$

we have

$$
\begin{aligned}
& D^{*} R_{2}(\varphi)\left(e_{i}^{k}, e_{j}^{k}\right) \\
= & \sum_{\substack{l, m, p, q \\
p^{\prime}, q^{\prime}, q}} A_{l, m, p, q, p^{\prime}, q^{\prime}}^{k}\left(\bar{e}_{l}^{k} \bar{e}_{m}^{k} \varphi_{p, q}^{k}\right) \cdot \varphi_{p^{\prime}, q^{\prime}}^{k} \\
+ & \sum_{\substack{l, m, p, q, q \\
p^{\prime}, q^{\prime}}} B_{l, m, p, q, p^{\prime}, q^{\prime}}^{k}\left(\bar{e}_{l}^{k} \varphi_{p, q}^{k}\right)\left(\bar{e}_{m}^{k} \varphi_{p^{\prime}, q^{\prime}}^{k}\right)+\sum_{\substack{l, p, q \\
p^{\prime}, q^{\prime}}} C_{l, p, q, p^{\prime}, q^{\prime}}^{k, i}\left(\bar{e}_{l}^{k} \varphi_{p, q}^{k}\right) \varphi_{p^{\prime}, q^{\prime}}^{k^{\prime}}
\end{aligned}
$$

where $A_{l, m, p, q, p^{\prime}, q^{\prime}}^{k} B_{l, m, p, q, p^{\prime}, q^{\prime}}^{k}$ and $C_{l, p, q, p^{\prime}, q^{\prime}}^{k, i}$ denote $C^{\infty}$ functions on $U_{k}$.

Therefore if $m \geq n$, Proposition 3.12 is proven. $\left(\operatorname{dim}_{R} M=2 n-1\right)$

Proposition 3.13. Assuming that $(M, \xi)$ is a compact normal s.p.c. manifold and $\operatorname{dim}_{R} M=2 n-1 \geq 7$, the following estimate holds uniformly for all $\varphi$ in $\Gamma\left(M,{ }^{\circ} \bar{T}^{\prime \prime} \otimes\left({ }^{\circ} T^{\prime \prime}\right)^{*}\right)$.

$$
\left\|N D_{1}^{*} R_{2}(\varphi)\right\|_{(m)}^{\prime \prime} \leqq\|\varphi\|_{(m)}^{\prime \prime 2}
$$

Proof. In Proposition 3.9, we have

$$
\|N \mu\|_{(m)}^{\prime \prime} \leqq\|\mu\|_{(m)}
$$

uniformly for $\mu$ in $\Gamma\left(M,{ }^{\circ} \bar{T}^{\prime \prime} \otimes\left({ }^{0} T^{\prime \prime}\right)^{*}\right)$. Setting $\mu=D^{*} R_{2}(\varphi)$ in this estimate, we have

$$
\left\|N D^{*} R_{2}(\varphi)\right\|_{(m)}^{\prime \prime} \leqq\left\|D^{*} R_{2}(\varphi)\right\|_{(m)} \text {. }
$$

From Proposition 3.12 and this estimate, we have Proposition 3.13.

Q. E. D. 


\section{§4. The Space $Z_{D_{i}}$ and the Closedness of $L Z_{D_{1}}$}

In this section we shall study the linear operator $L$ introduced in $\S 1$. We denote by $\Gamma_{(m)}^{\prime \prime}\left(M,{ }^{0} \bar{T}^{\prime \prime} \otimes\left({ }^{0} T^{\prime \prime}\right)^{*}\right) \quad\left(\operatorname{resp} . \Gamma_{(m)}^{\prime \prime}\left(M, F \otimes{ }^{2}\left({ }^{0} T^{\prime \prime}\right)^{*}\right)\right)$ the Hilbert space obtained by completing the pre-Hilbert space consisting of ${ }^{\circ} \bar{T}^{\prime \prime} \otimes\left({ }^{0} T^{\prime \prime}\right)^{*}$-valued $C^{\infty}$ functions (resp. $F \otimes \bigwedge^{2}\left({ }^{0} T^{\prime \prime}\right)^{*}$ valued $C^{\infty}$ functions) by the norm \|\|$_{(m)}^{\prime \prime}$ introduced in $\S 3$. We put $Z_{D_{1}}=\left\{\varphi: \varphi=\left(H+D D^{*} N\right) \varphi, \varphi \in \Gamma_{(m)}^{\prime \prime}\left(M,{ }^{\circ} \bar{T}^{\prime \prime} \otimes\left({ }^{0} T^{\prime \prime}\right)^{*}\right)\right\}$.

Then we have the following proposition.

Proposition 4. 1. The set $L Z_{D_{1}}$ is closed in $\Gamma_{(m)}^{\prime \prime}\left(M, F \otimes \wedge^{2}\left({ }^{0} T^{\prime \prime}\right)^{*}\right)$.

To prove this proposition, we make some preparations.

Lemma 4. 2. The following diagram commutes.

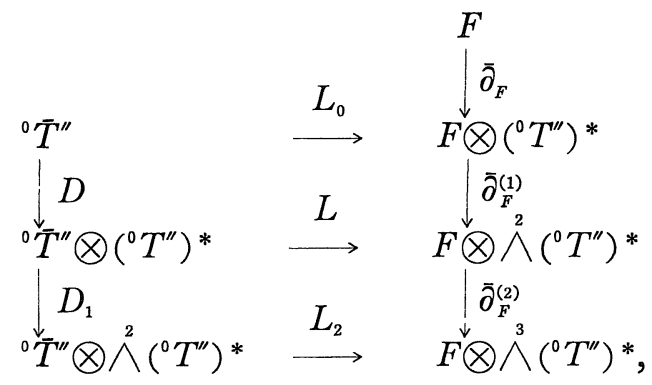

where we set $L_{0}$ as follows: for each $\theta$ in ${ }^{\circ} \bar{T}^{\prime \prime}$, we put

$$
L_{0} \theta(X)=-[X, \theta]_{F}
$$

where $X$ in ${ }^{\circ} T^{\prime \prime}$. And we set $L_{2}$ as follows: for each $\phi$ in ${ }^{\circ} \bar{T}^{\prime \prime} \otimes \wedge^{2}\left({ }^{0} T^{\prime \prime}\right)^{*}$, we put

$$
L_{2} \psi\left(X_{1}, X_{2}, X_{3}\right)=-\left(\bar{\partial}_{T^{\prime}}^{(2)} \psi\left(X_{1}, X_{2}, X_{3}\right)\right)^{F},
$$

where by $\left(\bar{\partial}_{T^{\prime}}^{(2)} \psi\left(X_{1}, X_{2}, X_{3}\right)\right)_{F}$ we denote the projection of $\bar{\partial}_{T^{\prime}}^{(2)} \psi\left(X_{1}, X_{2}, X_{3}\right)$ to $F$ according to the splitting $C \otimes T M={ }^{\circ} T^{\prime \prime} \oplus{ }^{\circ} \bar{T}^{\prime \prime} \oplus F$ introduced in $\S 1$. 
Proof. Of course the above maps $L_{0}, L_{2}$ are well defined. Especially $L_{0}$ is a $C^{\infty}$ bundle-isomorphism since $\left(M,{ }^{\circ} T^{\prime \prime}\right)$ is an s. p.c. Now we shall prove our lemma. For each $\theta \in \Gamma\left(M,{ }^{\circ} \bar{T}^{\prime \prime}\right)$, we have the relation:

$$
\begin{aligned}
& \left(\bar{\partial}_{F}^{(1)} \cdot L_{0} \theta\right)(X, Y) \\
& \quad=\left[X, L_{0} \theta(Y)\right]_{F}+\left[L_{0} \theta(X), Y\right]_{F}-L_{0} \theta([X, Y]) \\
& \quad=-\left[X,[Y, \theta]_{F}\right]_{F}-\left[[X, \theta]_{F}, Y\right]_{F}+[[X, Y], \theta]_{F},
\end{aligned}
$$

where $X, Y \in \Gamma\left(M,{ }^{\circ} T^{\prime \prime}\right)$. On the other hand

$$
\begin{aligned}
L D \theta(X, Y) & =[X, D \theta(Y)]_{F}+[D \theta(X), Y]_{F} \\
& =\left[X,[Y, \theta]_{0 T^{\prime \prime}}\right]_{F}+\left[[X, \theta]_{0 T^{\prime \prime}}, Y\right]_{F},
\end{aligned}
$$

where $X, Y \in \Gamma\left(M,{ }^{\circ} T^{\prime \prime}\right)$. From (4.1) and (4.2), we have the relation:

$$
\begin{aligned}
\left(\tilde{\partial}_{F}^{(1)} \cdot L_{0}-\right. & L \cdot D) \theta(X, Y) \\
= & -\left[X,[Y, \theta]_{F}\right]_{F}-\left[[X, \theta]_{F}, Y\right]_{F}+[[X, Y], \theta]_{F} \\
& -\left[X,[Y, \theta]_{O^{\prime \prime}}\right]_{F}-\left[[X, \theta]_{0 T^{\prime \prime}}, Y\right]_{F} \\
= & -\left[X,[Y, \theta]_{T^{\prime}}\right]_{F}-\left[[X, \theta]_{T^{\prime}}, Y\right]_{F}+[[X, Y], \theta]_{F} \\
= & -[X,[Y, \theta]]_{F}-[[X, \theta], Y]_{F}+[[X, Y], \theta]_{F} \\
= & 0,
\end{aligned}
$$

for any $X, Y \in \Gamma\left(M,{ }^{\circ} T^{\prime \prime}\right)$. Therefore we have

$$
\bar{\partial}_{F}^{(1)} \cdot L_{0}=L \cdot D \text {. }
$$

Similarly we have

$$
\bar{\partial}_{F}^{(2)} \cdot L=L_{2} \cdot D_{1} .
$$

Therefore we have our lemma.

Q. E. D.

Next we shall prove that $Z_{D_{1}}$ is closed in $\Gamma_{(m)}^{\prime \prime}\left(M,{ }^{\circ} \bar{T}^{\prime \prime} \otimes\left({ }^{0} T^{\prime \prime}\right)^{*}\right)$. The linear operator $D^{*} D N$ is a continuous operator from $\Gamma_{(m)}^{\prime \prime}(M$, $\left.{ }^{\circ} \bar{T}^{\prime \prime} \otimes\left({ }^{\circ} T^{\prime \prime}\right)^{*}\right)$ to itself. (In fact the following relation assures this.

$$
\left\|D^{*} D N \varphi\right\|_{(m)}^{\prime \prime}=\left\|N D^{*} D \varphi\right\|_{(m)}^{\prime \prime} \leq C|| D^{*} D \varphi\left\|_{(m)} \leq C^{\prime}|| \varphi\right\|_{(m)}^{\prime \prime},
$$

for all $\varphi$ in $\Gamma_{(m)}^{\prime \prime}\left(M,{ }^{0} \bar{T}^{\prime \prime} \otimes\left({ }^{0} T^{\prime \prime}\right)^{*}\right)$.) Therefore $\operatorname{Ker} D^{*} D N=Z_{D_{1}}$ is closed in $\Gamma_{(m)}^{\prime \prime}\left(M,{ }^{\circ} \bar{T}^{\prime \prime} \otimes\left({ }^{0} T^{\prime \prime}\right)^{*}\right)$. 
Similarly we have the following:

The set $Z_{\dot{f}_{F}^{(2)}}=\left\{\varphi: \varphi=\left(H_{F}+\bar{\partial}_{F}^{(1)} \bar{\partial}_{F}^{(1) *} N_{F}\right) \varphi, \varphi \in \Gamma_{(m)}^{\prime \prime}\left(M, F \otimes \bigwedge^{2}\left({ }^{0} T^{\prime \prime}\right)^{*}\right\} \quad\right.$ is closed in $\Gamma_{(m)}^{\prime \prime}\left(M, F \otimes \wedge^{2}\left({ }^{\circ} T^{\prime \prime}\right)^{*}\right)$.

Now we shall prove Proposition 4.1. It suffices to prove the following relation:

(4.3) $L D D^{*} N{\Gamma_{(m)}^{\prime \prime}}\left(M,{ }^{0} \bar{T}^{\prime \prime} \otimes\left({ }^{0} T^{\prime \prime}\right)^{*}\right)=\bar{\partial}_{F}^{(1)} \bar{\partial}_{F}^{(1) *} N_{F} \Gamma_{(m)}^{\prime \prime}\left(M, F \otimes \wedge^{2}\left({ }^{0} T^{\prime \prime}\right)^{*}\right)$, since $H \Gamma_{(m)}^{\prime \prime}\left(M,{ }^{0} \bar{T}^{\prime \prime} \otimes\left({ }^{\circ} T^{\prime \prime}\right)^{*}\right)$ is a finite dimensional vector space and $L Z_{D_{1}}$ is spanned by $L H \Gamma_{(m)}^{\prime \prime}\left(M,{ }^{\circ} \bar{T}^{\prime \prime} \otimes\left({ }^{0} T^{\prime \prime}\right)^{*}\right)$ and $L D D^{*} N \Gamma_{(m)}^{\prime \prime}\left(M, \Gamma^{0} \bar{T}^{\prime \prime} \otimes\right.$ $\left.\left({ }^{0} T^{\prime \prime}\right)^{*}\right)$. In fact the set $\bar{\partial}_{F}^{(1)} \bar{\partial}_{F}^{(1) *} N_{F} \Gamma_{(m)}^{\prime \prime}\left(M, F \otimes \wedge^{2}\left({ }^{0} T^{\prime \prime}\right)^{*}\right)$ is closed in $\Gamma_{(m)}^{\prime \prime}\left(M, F \otimes \wedge^{2}\left({ }^{0} T^{\prime \prime}\right)^{*}\right)$ (because of the relation:

$$
\left.\bar{\partial}_{F}^{(1)} \bar{\partial}_{F}^{(1) *} N_{F} \Gamma_{(m)}^{\prime \prime}\left(M, F \otimes \wedge^{2}\left({ }^{0} T^{\prime \prime}\right)^{*}\right)=Z_{\delta_{F}^{(2)}} \cap \operatorname{Ker} H_{F} \cdot\right)
$$

Now we shall prove (4.3). For any $\varphi$ in $\Gamma_{(m)}^{\prime \prime}\left(M,{ }^{0} \bar{T}^{\prime \prime} \otimes\left({ }^{0} T^{\prime \prime}\right)^{*}\right)$, we have that

$$
\left\|D D^{*} N \varphi\right\|_{(m)}^{\prime \prime}=\left\|N D D^{*} \varphi\right\|_{(m)}^{\prime \prime} \leq C\left\|D D^{*} \varphi\right\|_{(m)} \leq C^{\prime}\|\varphi\|_{(m)}^{\prime \prime} .
$$

And so

$$
\left\|L D D^{*} N \varphi\right\|_{(m)}^{\prime \prime} \leq C^{\prime \prime}|| D D^{*} N \varphi\left\|_{(m)}^{\prime \prime} \leq C^{\prime} C^{\prime \prime}\right\| \varphi \|_{(m)}^{\prime \prime}
$$

Hence

$$
L D D^{*} N \varphi \in \Gamma_{(m)}^{\prime \prime}\left(M, F \otimes \bigwedge^{2}\left({ }^{0} T^{\prime \prime}\right)^{*}\right) .
$$

On the other hand, it follows from Lemma 4.2 that

$$
\begin{aligned}
L D D^{*} N \varphi & =\left(H_{F}+\bar{\partial}_{F}^{(1)} \bar{\partial}_{F}^{(1) *} N_{F}+\bar{\partial}_{F}^{(2) *} \bar{\partial}_{F}^{(2)} N_{F}\right) L D D^{*} N \varphi \\
& =\bar{\partial}_{F}^{(1)} \bar{\partial}_{F}^{(1) *} N_{F} L D D^{*} N \varphi .
\end{aligned}
$$

Hence

$$
L D D^{*} N \Gamma_{(m)}^{\prime \prime}\left(M,{ }^{0} \bar{T}^{\prime \prime} \otimes\left({ }^{0} T^{\prime \prime}\right)^{*}\right) \subset \bar{\partial}_{F}^{(1)} \bar{\partial}_{F}^{(1) *} N_{F} \Gamma_{(m)}^{\prime \prime}\left(M,{ }^{0} \bar{T}^{\prime \prime} \otimes\left({ }^{0} T^{\prime \prime}\right)^{*}\right) .
$$

Conversely for any $\psi$ in $\Gamma_{(m)}^{\prime \prime}\left(M, F \otimes \wedge^{2}\left({ }^{0} T^{\prime \prime}\right)^{*}\right)$, we have that

$$
\begin{aligned}
\bar{\partial}_{F}^{(1)} \bar{\partial}_{F}^{(1) *} N_{F} \psi & =\bar{\partial}_{F}^{(1)} L_{0} \cdot L_{0}^{-1} \bar{\partial}_{F}^{(1) *} N_{F} \psi=L D \cdot L_{0}^{-1} \bar{\partial}_{F}^{(1) *} N_{F} \psi \\
& =L \cdot D D^{*} N D\left(L_{0}^{-1} \bar{\partial}_{F}^{(1) *} N_{F} \psi\right)=L \cdot D D^{*} N\left(D \cdot L_{0}^{-1} \cdot \bar{\partial}_{F}^{(1) *} N_{F} \psi\right) .
\end{aligned}
$$

Thus, if we have the relation

$$
\left\|D \cdot L_{0}^{-1} \bar{\partial}_{F}^{(1) *} N_{F} \psi\right\|_{(m)}^{\prime \prime} \leq C\|\psi\| \|_{(m)}^{\prime \prime}
$$


uniformly for all $\psi$ in $\Gamma_{(m)}^{\prime \prime}\left(M, F \otimes \bigwedge^{2}\left({ }^{0} T^{\prime \prime}\right)^{*}\right)$, our Proposition 4.1 is proven.

So it remains to prove (4.4). Let $\left\{U_{k}\right\}_{k \in K}$ be a system of finite coordinate covering of $M$ and $\left\{e_{i}^{k}\right\}_{i=1,2, \ldots, n-1}$ be a moving frame of ${ }^{\circ} T^{\prime \prime}$ on $U_{k}$. For each $\phi$ in $\Gamma_{(m)}^{\prime \prime}\left(M, F \otimes \bigwedge^{2}\left({ }^{0} T^{\prime \prime}\right)^{*}\right)$ we define $C^{\infty}$ functions $\left(N_{F} \bar{\partial}_{F}^{(1) *} \psi\right)_{i}^{k}$ on $U_{k}$ by the following formula:

$$
\left(N_{F} \bar{\partial}_{F}^{(1) *} \psi\right)_{i}^{k} \xi=\left(N_{F} \bar{\partial}_{F}^{(1) *} \psi\right)\left(e_{i}^{k}\right) .
$$

Let $\Gamma_{0}\left(U_{k}, F \otimes\left({ }^{0} T^{\prime \prime}\right)^{*}\right)$ be the space consisting of $F \otimes\left({ }^{0} T^{\prime \prime}\right)^{*}$-valued $C^{\infty}$ functions supported in $U_{k}$. For each $\varphi$ in $\Gamma_{0}\left(U_{k}, F \otimes\left({ }^{0} T^{\prime \prime}\right)^{*}\right)$, we set

$$
\left(e_{\alpha}^{k} \varphi\right)\left(e_{i}^{k}\right)=e_{\alpha}^{k} \varphi_{i}^{k}
$$

where $\varphi_{i}^{k}$ is defined by $\varphi_{i}^{k} \xi=\varphi\left(e_{i}^{k}\right)$. Similarly we set

$$
\left(\bar{e}_{\alpha}^{k} \varphi\right)\left(e_{i}^{k}\right)=\bar{e}_{\alpha}^{k} \varphi_{i}^{k}
$$

The differential operators $e_{\alpha}^{k}, \bar{e}_{\beta}^{k}$ can be extended to the differential operators on $\Gamma_{(m)}^{\prime \prime}\left(M, F \otimes\left({ }^{0} T^{\prime \prime}\right)^{*}\right)$ as follows :

Let $\rho_{k}$ be a $C^{\infty}$ function supported in $U_{k}$ such that

$$
\left.\rho_{k}\right|_{V_{k}}=1
$$

where $\left\{V_{k}\right\}_{k \in K}$ is a refinement of $\left\{U_{k}\right\}_{k \in K}$ such that $V_{k} \Subset U_{k}$. Then we define $e_{\alpha}^{k}$ as follows: for each $\varphi$ in $\Gamma\left(M, F \otimes\left({ }^{0} T^{\prime \prime}\right)^{*}\right)$,

$$
\left(e_{\alpha}^{k} \varphi\right)\left(e_{i}^{k}\right)=e_{\alpha}^{k}\left(\rho_{k} \varphi_{i}^{k}\right) .
$$

Similarly we define $\bar{e}_{\alpha}^{k}$ as follows: for each $\varphi$ in $\Gamma\left(M, F \otimes\left({ }^{0} T^{\prime \prime}\right)^{*}\right)$,

$$
\left(\bar{e}_{\alpha}^{k} \varphi\right)\left(e_{i}^{k}\right)=\bar{e}_{\alpha}^{k}\left(\rho_{k} \varphi_{i}^{k}\right) .
$$

Now to get (4.4), it suffices to prove the following:

$$
\left\|e_{\alpha}^{k} \bar{\partial}_{F}^{(1) *} N_{F} \psi\right\|_{(m)}^{\prime \prime} \leq C\|\phi\|_{(m)}^{\prime \prime}
$$

uniformly for all $\phi$ in $\Gamma_{(m)}^{\prime \prime}\left(M, F \otimes \wedge^{2}\left({ }^{0} T^{\prime \prime}\right)^{*}\right)$. With the above notations (4.5) can be written as follows.

$$
\begin{aligned}
& \left\|e_{\alpha}^{k} e_{\beta}^{k} e_{r}^{k} \bar{\partial}_{F}^{(1) *} N_{F} \psi\right\|_{(m)} \leq C\|\psi\|_{(m)}^{\prime \prime}, \\
& \left\|\bar{e}_{\alpha}^{k} e_{\beta}^{k} e_{r}^{k} \bar{\partial}_{F}^{(1) *} N_{F} \psi\right\|_{(m)} \leq C\|\psi\|_{(m)}^{\prime \prime}, \\
& \left\|e_{\alpha}^{k} \bar{e}_{\beta}^{k} e_{r}^{k} \bar{\partial}_{F}^{(1) *} N_{F} \psi\right\|_{(m)} \leq C\|\psi\|_{(m)}^{\prime \prime}
\end{aligned}
$$


and

$$
\left\|\bar{e}_{\alpha}^{k} \bar{e}_{\beta}^{k} e_{r}^{k} \bar{\partial}_{F}^{(1) *} N_{F} \psi\right\|_{(m)} \leq C\|\psi\|_{(m)}^{\prime \prime},
$$

for all $\alpha, \beta, \gamma=1,2, \ldots, n-1, k \in K$ and $\phi$ in $\Gamma_{(m)}^{\prime \prime}\left(M, F \otimes \wedge^{2}\left({ }^{0} T^{\prime \prime}\right)^{*}\right)$.

Here we shall prove only (4.6), the others being proven similarly. We fix $k$ once and for all and suppress the suffix $k$ of $\varphi \in \Gamma\left(M, F \otimes \wedge^{2}\right.$ $\left.\left({ }^{0} T^{\prime \prime}\right)^{*}\right)$ till the end of the proof and $e_{\alpha}^{k}=e_{\alpha}$.

First from Proposition 3.2 we have,

$$
\left\|e_{\alpha} \varphi\right\|_{(m)}^{2} \leq C\left\{\left\|\bar{\partial}_{F}^{(1)} \varphi\right\|_{(m)}^{2}+\left\|\bar{\partial}_{F}^{*} \varphi\right\|_{(m)}^{2}+\|\varphi\|_{(m)}^{2}\right\}
$$

for all $\varphi \in \Gamma\left(M, F \otimes\left({ }^{0} T^{\prime \prime}\right)^{*}\right)$. Then putting

$$
\varphi=e_{\beta} e_{r} N_{F} \bar{\partial}_{F}^{(1) *} \psi,
$$

where $\psi \in \Gamma\left(M, F \otimes \wedge^{2}\left({ }^{0} T^{\prime \prime}\right) *\right)$, this gives rise to the inequality

(4.10) $\quad\left\|e_{\alpha} e_{\beta} e_{r} N_{F} \bar{\partial}_{F}^{(1) *} \psi\right\|_{(m)}^{2}$

$$
\leq C\left\{\left\|\bar{\partial}_{F}^{(1)} e_{\beta} e_{r} N_{F} \bar{\partial}_{F}^{(1) *} \phi\right\|_{(m)}^{2}+\left\|\bar{\partial}_{F}^{*} e_{\beta} e_{r} N_{F} \bar{\partial}_{F}^{(1) *} \psi\right\|_{(m)}^{2}+\left\|e_{\beta} e_{r} N_{F} \bar{\partial}_{F}^{(1) *} \phi\right\|_{(m)}^{2}\right\},
$$

for all $\phi \in \Gamma\left(M, F \otimes \wedge^{2}\left({ }^{0} T^{\prime \prime}\right)^{*}\right)$. While

(4.11) $\quad \bar{\partial}_{F}^{(1)} e_{\beta} e_{r} N_{F} \bar{\partial}_{F}^{(1) *} \psi=e_{\beta} \bar{\partial}_{F}^{(1)} e_{r} N_{F} \bar{\partial}_{F}^{(1) *} \psi+\left[e_{\beta}, \bar{\partial}_{F}^{(1)}\right] e_{r} N_{F} \bar{\partial}_{F}^{(1) *} \psi$.

$\left[e_{\beta}, \bar{\partial}_{F}^{(1)}\right]$ has the estimate;

$$
\left\|\left[e_{\beta}, \bar{\partial}_{F}^{(1)}\right] \varphi\right\|_{(m)} \leq C\|\varphi\|_{(m)}^{\prime},
$$

$\varphi \in \Gamma\left(M, F \otimes\left({ }^{0} T^{\prime \prime}\right)^{*}\right)$. Hence

$$
\begin{aligned}
\left\|\left[e_{\beta}, \bar{\partial}_{F}^{(1)}\right] e_{r} N_{F} \bar{\partial}_{F}^{(1) *} \psi\right\|_{(m)} & \leq C\left\|e_{r} N_{F} \bar{\partial}_{F}^{(1) *} \phi\right\|_{(m)}^{\prime} \\
& \leq C^{\prime}\left\|N_{F} \bar{\partial}_{F}^{(1) *} \psi\right\|_{(m)}^{\prime \prime} \\
& \leq C^{\prime \prime}\left\|\bar{\partial}_{F}^{(1) *} \phi\right\|_{(m)} \\
& \leq C^{\prime \prime \prime}\|\phi\|_{(m)}^{\prime} \\
& \leq C^{\prime \prime \prime}\|\phi\|_{(m)}^{\prime \prime} .
\end{aligned}
$$

On the other hand, as for the first term of (4.11), we have

$$
e_{\beta} \bar{\partial}_{F}^{(1)} e_{r} N_{F} \bar{\partial}_{F}^{(1) *} \psi=e_{\beta} e_{r} \bar{\partial}_{F}^{(1)} N_{F} \bar{\partial}_{F}^{(1) *} \psi+e_{\beta}\left[\bar{\partial}_{F}^{(1)}, e_{r}\right] N_{F} \bar{\partial}_{F}^{(1) *} \phi .
$$

Then we get similarly

$$
\begin{aligned}
\left\|e_{\beta}\left[\bar{\partial}_{F}^{(1)}, e_{r}\right] N_{F} \bar{\partial}_{F}^{(1) *} \psi\right\|_{(m)} & \leq C\left\|N_{F} \bar{\partial}_{F}^{(1) *} \psi\right\|_{(m)}^{\prime \prime} \\
& \leq C^{\prime}\left\|\bar{\partial}_{F}^{(1) *} \psi\right\|_{(m)}^{\prime \prime} \\
& \leq C^{\prime \prime}\|\psi\|_{(m)}^{\prime}
\end{aligned}
$$




$$
\leq C^{\prime \prime \prime}\|\phi\|_{(m)}^{\prime \prime}
$$

and the first term becomes

$$
e_{\beta} e_{\tau} N_{F} \bar{\partial}_{F}^{(1)} \bar{\partial}_{F}^{(1) *} \phi
$$

and hence can be estimated as follows.

$$
\begin{aligned}
\left\|e_{\beta} e_{r} \bar{\partial}_{F}^{(1)} N_{F} \bar{\partial}_{F}^{(1) *} \phi\right\|_{(m)} & =\left\|e_{\beta} e_{\gamma} N_{F} \bar{\partial}_{F}^{(1)} \bar{\partial}_{F}^{(1) *} \psi\right\|_{(m)} \\
& \leq C \|\left.\bar{\partial}_{F}^{(1)} \bar{\partial}_{F}^{(1) *} \psi\right|_{,(m)} ^{\prime} \\
& \leq C^{\prime}|| \psi \mid \|_{(m)}^{\prime \prime} .
\end{aligned}
$$

Next we show that the second term of (4.10) can be estimated as follows:

$$
\left\|\bar{\partial}_{F}^{*} e_{\beta} e_{\gamma} N_{F} \bar{\partial}_{F}^{(1) *} \phi\right\|_{(m)}^{2} \leq C\|\phi\|_{(m)}^{\prime \prime}
$$

We note that

$$
\bar{\partial}_{F}^{*} e_{\beta} e_{r} N_{F} \bar{\partial}_{F}^{*} \psi=e_{\beta} \bar{\partial}_{F}^{*} e_{r} N_{F} \bar{\partial}_{F}^{*} \psi+\left[\bar{\partial}_{F}^{*}, e_{\beta}\right] e_{r} N_{F} \bar{\partial}_{F}^{*} \psi .
$$

The principal term of the differential operator $\left[\bar{\partial}_{F}^{*}, e_{\beta}\right]$ has the following form :

$$
\sum_{j} a_{j}^{\beta} e_{j}+\sum_{j} e_{j}^{\beta} \bar{e}_{j}+c_{j} L_{\xi},
$$

where $a_{j}^{\beta}$, $e_{j}^{\beta}$ and $c_{j}$ are $C^{\infty}$ functions supported in $U_{k}$ and $L_{\xi}$ is the Lie derivation defined in $\S 2$.

So we have

$$
\left\|\left[\bar{\partial}_{F}^{*}, e_{\beta}\right] e_{r} N_{F} \bar{\partial}_{F}^{*} \psi\right\|_{(m)} \leq C\left\|e_{r} N_{F} \bar{\partial}_{F}^{*} \phi\right\|_{(m)}^{\prime}+C^{\prime} \mid i L_{\xi} e_{r} N_{F} \bar{\partial}_{F}^{*} \phi \|_{(m)} .
$$

We estimate the latter term using the following relation:

$$
L_{\xi} e_{r} N_{F} \bar{\partial}_{F}^{*} \psi=e_{r} L_{\xi} N_{F} \bar{\partial}_{F}^{*} \psi+\left[L_{\xi}, e_{r}\right] N_{F} \bar{\partial}_{F}^{*} \psi \text {. }
$$

Since $(M, \xi)$ is a normal s. p. c., we have that the principal part of $\left[L_{\xi}, e_{\tau}\right]$ is $\sum_{j} a_{j}^{\prime} e_{j}$, where $a_{j}^{\prime}$ are $C^{\infty}$ functions supported in $U_{k}$. Then from Proposition 2.7.1 and Corollary 2.8.1, we have

$$
\begin{aligned}
& \left\|L_{\xi} e_{r} N_{F} \bar{\partial}_{F}^{(1) *} \psi\right\|\left\|_{(m)} \leq\right\| e_{r} L_{\xi} N_{F} \bar{\partial}_{F}^{(1) *} \psi_{i}\left\|_{(m)}+\right\|\left[L_{\xi}, e_{r}\right] N_{F} \bar{\partial}_{F}^{(1) *} \psi \|_{\left({ }^{\prime}(m)\right.} \\
& \leq\left\|e_{\tau} N_{F} L_{\xi} \bar{\partial}_{F}^{(1) *} \phi\right\|_{(m)}+C\left\|N_{F} \bar{\partial}_{F}^{(1) *} \phi\right\|_{(m)}^{\prime} \\
& \leq C^{\prime \prime}\left\|e_{r} \bar{\partial}_{F}^{(1) *} N_{F} L_{\xi} \psi\right\|_{(m)}+C^{\prime}\|\phi\|_{(m)}^{\prime} \text {. }
\end{aligned}
$$

While from the estimate 


$$
\begin{aligned}
\| e_{\tau}\left(\bar{\partial}_{F}^{(1) *}\right. & \left.N_{F} L_{\xi} \psi\right) \|_{(m)} \leq C\left\{\left\|\bar{\partial}_{F}^{(1)} \bar{\partial}_{F}^{(1) *} N_{F} L_{\xi} \psi\right\|_{(m)}\right. \\
& \left.+\left\|\bar{\partial}_{F}^{(2) *} \bar{\partial}_{F}^{(1) *} N_{F} L_{\xi} \psi\right\|_{(m)}+\left\|\bar{\partial}_{F}^{(1) *} N_{F} L_{\xi} \psi_{l}\right\|_{(m)}\right\},
\end{aligned}
$$

we have

$$
\left\|e_{\gamma}\left(\bar{\partial}_{F}^{(1) *} N_{F} L_{\xi} \psi\right)\right\|_{(m)} \leq C\left\|L_{\xi} \psi\right\|_{(m)} .
$$

So it follows that

$$
\left\|L_{\xi} e_{\gamma} N_{F} \bar{\partial}_{F}^{(1) *} \phi\right\|_{(m)} \leq C\|\phi\|_{(m)}^{\prime \prime} \text {. }
$$

And the second term of (4.12) can be estimated by the same method as above.

Then we get the relation:

$$
\left\|e_{\alpha} e_{\beta} e_{r} N_{F} \bar{\partial}_{F}^{(1) *} \phi\right\|_{(m)} \leq C\|\psi\|_{(m)}^{\prime \prime} \text {. }
$$

Therefore Proposition 4. 1 is proven.

Q. E. D.

Remark. For all $\varphi \in \Gamma_{(m)}^{\prime \prime}\left(M, F \otimes \wedge^{2}\left({ }^{0} T^{\prime \prime}\right)^{*}\right)$ with $\bar{\delta}_{F}^{(2)} \varphi=0$ and $H_{F} \varphi=0$, we have that

$$
\varphi \in L Z_{D_{1}}
$$

Proof. From the assumptions $\bar{\partial}_{F}^{(2)} \varphi=0$ and $H_{F} \varphi=0$, we have the relation :

$$
\varphi=\bar{\partial}_{F}^{(1)} \bar{\partial}_{F}^{(1)} * N_{F} \psi
$$

by Hodge decomposition. Moreover since $\varphi \in \Gamma_{(m)}^{\prime \prime}\left(M, F \otimes \wedge^{2}\left({ }^{0} T^{\prime \prime}\right)^{*}\right)$ we have that

$$
\varphi \in L Z_{D_{1}}
$$

from the proof of Proposition 4. 1.

Proposition 4.2. There exists a closed subspace $C_{2}$ of $Z_{D_{1}}$ such that $\left.L\right|_{c_{2}}$ is an isomorphism from $C_{2}$ to $L C_{2}=L Z_{D_{1}}$, where $\left.L\right|_{c_{2}}$ denotes the restriction of $L$ to $C_{2}$.

Proof. We set $C_{1}=\operatorname{Ker} L \cap Z_{D_{1}}$ and we shall define a closed subspace :

$$
C_{2}=\left\{x: x \in Z_{D_{1}},(x, y)=0 \text { for all } y \in C_{1}\right\}
$$


where $($,$) means the inner product defined by the \|,\|_{(m)}^{\prime \prime}$ norm. Then we have the orthogonal decomposition of the Hilbert space:

$$
Z_{D_{1}}=C_{1} \oplus C_{2}
$$

and $\left.L\right|_{c_{2}}$ is an isomorphism.

Q. E. D.

By $A: L Z_{D_{1}} \rightarrow C_{2}$ we denote the inverse of $\left.L\right|_{c_{2}}$. This map $A$ plays an essential role in our construction of versal family of isolated singularity.

\section{§. Construction of the Versal Family}

In $\S 3$, we formed the adjoint operator $\bar{\partial}_{T^{\prime}}^{(i) *}$ of $\bar{\partial}_{T^{\prime}}^{(i)}$ and set up the Laplacian

$$
\square=\bar{\partial}_{T^{\prime}}^{(i-1)} \cdot \bar{\partial}_{T^{\prime}}^{*(i-1)}+\bar{\partial}_{T^{\prime}}^{*(i)} \cdot \bar{\partial}_{T^{\prime \prime}}^{(i)}
$$

As is usual in the theory of harmonic forms for the sub-elliptic differential operator $\square$, we have the harmonic space $H_{T^{\prime}}^{(1)}$ in $\Gamma(M$, $\left.T^{\prime} \otimes\left({ }^{0} T^{\prime \prime}\right)^{*}\right)$. We shall introduce a first order differential operator $\mathscr{L}$ from $\Gamma\left(M, T^{\prime} \otimes\left({ }^{0} T^{\prime \prime}\right)^{*}\right)$ to $\Gamma\left(M,{ }^{\circ} \bar{T}^{\prime \prime} \otimes\left({ }^{0} T^{\prime \prime}\right)^{*}\right)$ as follows: For each $\psi$ in $\Gamma\left(M, T^{\prime} \otimes\left({ }^{0} T^{\prime \prime}\right) *\right)$, we put

$$
\mathscr{L} \psi=\psi-\bar{\partial}_{T}, \theta_{\psi},
$$

where $\theta_{\phi}$ is in $\Gamma\left(M,{ }^{\circ} \bar{T}^{\prime \prime}\right)$ such that

$$
\left[X, \theta_{\psi}\right]_{F}=\psi(X)_{F},
$$

for any $X$ in $\Gamma\left(M,{ }^{\circ} T^{\prime \prime}\right)$. With the above situation, we have the following Proposition.

Proposition 5.1. The map $\mathscr{L} \mid H_{T^{\prime}}^{(1)}$, being restricted to $H_{T^{\prime}}^{(1)}$ is injective.

Proof. We assume that $\mathscr{L} \varphi=0$ and $\varphi \in H_{T^{\prime}}^{(1)}$ From the definition of $\mathscr{L}$, we rewrite these relations as follows

$$
\varphi-\bar{\partial}_{T^{\prime}} \theta_{\varphi}=0
$$

and 


$$
\varphi \in H_{T^{\prime}}^{(1)}
$$

So we have that

$$
\varphi=0 \text {. }
$$

Therefore we have our Proposition.

Q. E. D.

We use the notation $\mathscr{H}$ for $\mathscr{L}\left(H_{T^{\prime}}^{(1)}\right)$. The Main Theorem in this paper is the following.

Theorem 5. 2. Under the assumption $H_{T^{\prime}}^{(2)}=0, \operatorname{dim}_{R} M=2 n-1 \geq 7$ and that $(M, \xi)$ is a normal s.p.c. manifold, there exists a deformation $\left(M,{ }^{(t)} T^{\prime \prime}\right)$ which is parametriqed complex analitically by a neighborhood $U$ of the origin in the euclidean space $\mathscr{H}$ such that the following relations $A .0$ ), A.1) and $A .2$ ) hold.

A. 0) $\varphi(0)=0$.

A. 1) For every $t \in U, \varphi(t) \in \Gamma_{(m)}^{\prime \prime}\left(M,{ }^{0} \bar{T}^{\prime \prime} \otimes\left({ }^{0} T^{\prime \prime}\right)^{*}\right)$ satisfies

$$
P(\varphi(t))=0 \text {. }
$$

A. 2) The linear term of $\varphi\left(t_{1}, t_{2}, \ldots, t_{q}\right)$ is equal to $\sum_{i=1}^{q} \beta_{q} t_{q}$, where $\left\{\beta_{\lambda}\right\}_{1 \leq \lambda \leq q}$ is a basis of $\mathscr{H}$, where $q=\operatorname{dim} \mathscr{H}$ and $\left(t_{1}, t_{2}, \ldots, t_{q}\right)$ are local coordinate of $U$.

Here $m$ is a sufficiently large integer, in particular $m \geq n+2$.

Proof. We shall prove this theorem by using Kodaira-Spencer's methods [8] and the a priori estimates in $\S 3$. Let $\varphi(t)$ be a $\Gamma\left(M,{ }^{\circ} \bar{T}^{\prime \prime} \otimes\left({ }^{0} T^{\prime \prime}\right) *\right)$ valued holomorphic function and

$$
\varphi(t)=\sum \varphi_{k_{1} \ldots k_{q}} t_{1}^{k_{1}} \ldots t_{q}^{k_{q}}
$$

be the power series expansion of $\varphi(t)$ with $\varphi(0)=0$. For simplicity, we abbreviate

$$
\varphi(t)=\sum_{\mu=1}^{\infty} \varphi_{\mu}(t)
$$

where $\varphi_{\mu}(t)$ is a homogeneous polynomial of degree $\mu$ in $\left(t_{1}, \ldots, t_{q}\right)$. 
Let

$$
\varphi^{\mu}(t)=\sum_{\lambda=1}^{\mu} \varphi_{\lambda}(t) .
$$

For any $\Gamma\left(M,{ }^{\circ} \bar{T}^{\prime \prime} \otimes\left({ }^{0} T^{\prime \prime}\right)^{*}\right)$ valued holomorphic functions $\varphi(t)$, $\psi(t)$ in $\left(t_{1}, \ldots, t_{q}\right)$, we indicate by $\varphi(t) \equiv \psi(t)$ that the power series expansion of $\varphi(t)-\phi(t)$ in $\left(t_{1}, \ldots, t_{q}\right)$ contains no terms of degree less than $\mu$.

Glearly A. 0) and A. 1) is equivalent to the system of congruence :

$$
D_{1} \varphi^{\mu}(t)+R_{2}\left(\varphi^{\mu}(t)\right) \underset{\mu+1}{\equiv} 0
$$

and

$$
L \varphi^{\mu}(t) \underset{\mu+1}{\equiv 0}
$$

where $\varphi^{\mu}(t) \in \Gamma_{(m)}^{\prime \prime}\left(M,{ }^{0} \bar{T}^{\prime \prime} \otimes\left({ }^{0} T^{\prime \prime}\right)^{*}\right) \quad(\mu=1,2, \ldots)$. Since $R_{2}(\varphi)$ is a second order polynomial with respect to $\varphi$, we have

$$
R_{2}\left(\varphi^{\mu}(t)\right) \underset{\mu+1}{\equiv} R_{2}\left(\varphi^{\mu-1}(t)\right) .
$$

Hence we may rewrite (5.1) ${ }_{\mu}$ as follows.

$$
D_{1} \varphi^{\mu}(t)+R_{2}\left(\varphi^{\mu-1}(t)\right) \underset{\mu+1}{\equiv} 0
$$

and

$$
L \varphi^{\mu}(t)=0
$$

Since

$$
D_{1} \varphi^{\mu}(t)=D_{1} \varphi_{\mu}(t)+D_{1} \varphi^{\mu-1}(t)
$$

and

$$
P\left(\varphi^{\mu-1}(t)\right)=D_{1} \varphi^{\mu-1}(t)+R_{2}\left(\varphi^{\mu-1}(t)\right),
$$

where

$$
\varphi^{\mu}(t) \in \Gamma_{(m)}^{\prime \prime}\left(M,{ }^{0} \bar{T}^{\prime \prime} \otimes\left({ }^{0} T^{\prime \prime}\right)^{*}\right),
$$

these are equivalent to the following:

$$
\text { (5. 4. 1) }{ }_{\mu} \quad D_{1} \varphi_{\mu}(t)+P\left(\varphi^{\mu-1}(t)\right) \underset{\mu+1}{\equiv 0,}
$$

and 
(5. 4. 2) $)_{\mu}$

$$
L \varphi_{\mu}(t)=0
$$

with $\varphi_{\mu}(t) \in \Gamma_{(m)}^{\prime \prime}\left(M,{ }^{0} \bar{T}^{\prime \prime} \otimes\left({ }^{0} T^{\prime \prime}\right)^{*}\right)(\mu=1,2, \ldots)$.

Now we shall construct $\varphi_{\mu}(t)$ by induction of $\mu$. We set $\varphi_{0}=0$ and prove that $\varphi_{1}(t)=\sum_{\lambda=1}^{q} \beta_{\lambda} t_{\lambda}$ satisfies (5.4.1) $)_{\mu}$ and (5.4.2) $)_{\mu}$, where $\left\{\beta_{\lambda}\right\}$ is a basis of $\mathscr{H}$.

In fact from the defininition of $\mathscr{H}$ : for each $\varphi \in \mathscr{H}, \bar{\partial}_{T^{\prime}}^{(1)} \varphi=0$ and $\varphi \in \Gamma\left(M,{ }^{\circ} \bar{T}^{\prime \prime} \otimes\left({ }^{0} T^{\prime \prime}\right)^{*}\right)$. So we have that for each $\varphi \in \mathscr{H}, D_{1} \varphi=0$ and $L \varphi=0$.

Suppose that $\varphi^{\mu-1}(t)$ satisfying (5.4.1) ${ }_{\mu}$ and (5.4.2) ${ }_{\mu}$ are already determined. We want to define a homogeneous polynomial $\varphi_{\mu}(t)$ of degree $\mu$ in $\left(t_{1}, \ldots, t_{q}\right)$. For this first we shall study the following differential equation:

$$
\bar{\partial}_{T^{\prime}}^{(1)} \psi(t)+P\left(\varphi^{\mu-1}(t)\right) \underset{\mu+1}{\equiv 0} .
$$

Under our assumptions $H_{T^{\prime}}^{(2)}=0$ and $\operatorname{dim}_{R} M=2 n-1 \geq 7$, we shall show that the partial differential equation (5.5) has a solution. We shall recall a result obtained in [1].

Lemma 5. 3. For any element $\varphi$ in $\Gamma\left(M, T^{\prime} \otimes\left({ }^{0} T^{\prime \prime}\right)^{*}\right)$,

$$
\bar{\partial}_{T^{\prime}} P(\varphi)=0 .
$$

For the proof see Theorem 3.10 in [1]. This lemma holds for any 2 times continuously differentiable $\varphi$. Therefore we may assume that for each $\varphi \in \Gamma_{(m)}^{\prime \prime}\left(M,{ }^{\circ} \bar{T}^{\prime \prime} \otimes\left({ }^{0} T^{\prime \prime}\right)^{*}\right)$

$$
\bar{\partial}_{T}^{\varphi}, P(\varphi)=0
$$

From the assumption $P\left(\varphi^{\mu-1}(t)\right) \underset{\mu}{\equiv}$, we have

$$
\bar{\partial}_{T^{\prime}}^{(2)}\left(P\left(\varphi^{\mu-1}(t)\right)\right) \underset{\mu+1}{\equiv} \bar{\partial}_{T^{\prime}} \varphi^{\mu-1}(t)\left(P\left(\varphi^{\mu-1}(t)\right)\right)=0
$$

(Lemma 5.3).

We put $\psi(t)$ as follows:

$$
\psi(t)=-\bar{\partial}_{T^{\prime}}^{*} N_{T^{\prime}} P\left(\varphi^{\mu-1}(t)\right) .
$$

Then from the assumption $H_{T^{\prime}}^{(2)}=0$ and (5.6), $\psi(t)$ satisfies (5.5). Hence $\mathscr{L} \psi(t)$ also satisfies (5.5). Namely 


$$
\bar{\partial}_{T^{\prime}}^{(1)} \mathscr{L} \psi(t)+P\left(\varphi^{\mu-1}(t)\right) \underset{\mu+1}{\equiv 0} .
$$

Noting that $\mathscr{L} \psi(t)$ is a $\left.{ }^{0} \bar{T}^{\prime \prime} \otimes\left({ }^{0} T^{\prime \prime}\right)^{*}\right)$-valued global form, we rewrite this relation according to the splitting $T^{\prime}={ }^{0} \bar{T}^{\prime \prime} \oplus F$ as follows.

$$
\begin{gathered}
D_{1} \mathscr{L} \psi(t)+P\left(\varphi^{\mu-1}(t)\right) \underset{\mu+1}{\equiv 0}, \\
L \mathscr{L} \phi(t)=0 .
\end{gathered}
$$

From these, we have

$$
\begin{gathered}
N D_{1}^{*} D_{1} \mathscr{L} \phi(t)+N D_{1}^{*} P\left(\varphi^{\mu-1}(t)\right) \underset{\mu+1}{\equiv} 0, \\
L \mathscr{L} \phi(t)=0
\end{gathered}
$$

which in turn imply

$$
L N D^{*} P\left(\varphi^{\mu-1}(t)\right) \underset{\mu+1}{\equiv} L\left(H \mathscr{L} \psi(t)+D_{1} D_{1}^{*} N \mathscr{L} \psi(t)\right),
$$

where $H$ denotes the harmonic operator for

$$
\square=D \cdot D^{*}+D_{1}^{*} \cdot D_{1}
$$

From (5.11), it follows that the $\mu$-th homogeneous term of $L N D_{1}^{*} P\left(\varphi^{\mu-1}(t)\right)$ is in $L Z_{D_{1}}$, where $Z_{D_{1}}$ is the sub-space of $\Gamma_{(m)}^{\prime \prime}(M$, $\left.{ }^{\circ} \bar{T}^{\prime \prime} \otimes\left({ }^{0} T^{\prime \prime}\right)^{*}\right)$ introduced in $\S 4$. In fact from (5.11), we have the relation :

$$
L N D^{*} P\left(\varphi^{\mu-1}(t)\right)-L H \mathscr{L} \psi(t) \underset{\mu+1}{\equiv} L D D^{*} N \mathscr{L} \psi(t) .
$$

Therefore it follows that

$$
H_{F}\left\{L N D^{*} P\left(\varphi^{\mu-1}(t)\right)-L H \mathscr{L} \psi(t)\right\} \underset{\mu+1}{\equiv 0}
$$

and

$$
\bar{\partial}_{F}\left\{L N D_{1}^{*} P\left(\varphi^{\mu-1}(t)\right)-L H \mathscr{L} \psi(t)\right\} \underset{\mu+1}{\equiv 0} .
$$

from Lemma 4.2. On the other hand the following estimates hold.

$$
\begin{array}{ll}
\left\|L N D_{1}^{*} P\left(\varphi^{\mu-1}(t)\right)\right\|_{(m)}^{\prime \prime} & \\
\leq C_{1}\left\|N D^{*} P\left(\varphi^{\mu-1}(t)\right)\right\|_{(m)}^{\prime \prime} & \text { (by continuity of } L \text { ) } \\
\leq C_{2}\left\|D_{1}^{*} P\left(\varphi^{\mu-1}(t)\right)\right\|_{(m)} & \text { (by Proposition } 3.9) \\
\leq C_{3}\left\|\varphi^{\mu-1}(t)\right\|_{(m)}^{\prime \prime 2} & \text { (by Proposition 3. 12) }
\end{array}
$$

for sufficiently small $t$. Since $L H \mathscr{L} \phi(t)$ is a $C^{\infty}$ form, we have the 
above from the remark of Proposition 4.1.

Now we define a homogeneous polynomial $\varphi_{\mu}(t)$ of degree $\mu$ as follows :

$$
\varphi_{\mu}(t) \underset{\mu+1}{\equiv}-N D_{1}^{*} P\left(\varphi^{\mu-1}(t)\right)+A\left(L N D_{1}^{*} P\left(\varphi^{\mu-1}(t)\right)\right),
$$

where $A$ denotes the linear operator introduced at the end of $\S 4$.

Then $\varphi_{\mu}(t)$ satisfies the following equation.

$$
\begin{aligned}
D_{1} \varphi_{\mu}(t) & \equiv-D_{1} N D_{1}^{*} P\left(\varphi^{\mu-1}(t)\right) \\
& \underset{\mu+1}{\equiv}-P\left(\varphi^{\mu-1}(t)\right)+D_{2}^{*} D_{2} N P\left(\varphi^{\mu-1}(t)\right) .
\end{aligned}
$$

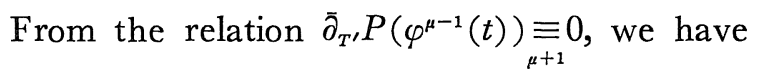

$$
\begin{aligned}
D_{2} P\left(\varphi^{\mu-1}(t)\right) & =\left(\bar{\partial}_{T^{\prime}}^{(2)} P\left(\varphi^{\mu-1}(t)\right)\right)_{0_{T^{\prime \prime}}} \\
& \equiv 0 . \\
\underset{\mu+1}{\equiv} &
\end{aligned}
$$

From (5.13) and (5. 14), it follows that

$$
D_{1} \varphi_{\mu}(t) \underset{\mu-1}{\equiv}-P\left(\varphi^{\mu-1}(t)\right)
$$

while from (5.12), we have

$$
\begin{aligned}
L \varphi_{\mu}(t) \equiv & \equiv-L N D_{1}^{*} P\left(\varphi^{\mu-1}(t)\right)+L \cdot A\left(L N D_{1}^{*} P\left(\varphi^{\mu-1}(t)\right)\right) \\
& =0 .
\end{aligned}
$$

Note that $\varphi_{\mu}(t)$ is in $\Gamma_{(m)}^{\prime \prime}\left(M,{ }^{\circ} \bar{T}^{\prime \prime} \otimes\left({ }^{0} T^{\prime \prime}\right)^{*}\right)$. Hence this completes our inductive construction of $\varphi_{\mu}(t)$.

Now we shall prove that the power series

$$
\varphi(t)=\varphi_{1}(t)+\varphi_{2}(t)+\ldots+\varphi_{\mu}(t)+\ldots,
$$

converges in \|\|$_{(m)}^{\prime \prime}$-norm for small $t$.

Consider a power series

$$
f(t)=\sum f_{h_{1} \ldots h_{q}} t_{1}^{h_{1}} t_{2}^{h_{2}} \ldots t_{q}^{h_{q}}
$$

whose coefficients $f_{h_{1} \ldots h_{q}}$ are in $\Gamma\left(M,{ }^{0} \bar{T}^{\prime \prime} \otimes\left({ }^{0} T^{\prime \prime}\right)^{*}\right)$ and a power series

$$
a(t)=\sum a_{h_{1} \ldots h_{q}} t_{1}^{h_{1}} t_{2}^{h_{2}} \ldots t_{q}^{h_{q}}
$$


with non-negative coefficients. By writing $f(t) \ll a(t)$ we indicate that

$$
\left\|f_{h_{1} \ldots h_{q}}\right\|_{(m)}^{\prime \prime} \leq a_{h_{1} \ldots h_{q}}
$$

for all $\left(h_{1} \ldots h_{q}\right)$. For each $\Gamma\left(M,{ }^{\circ} \bar{T}^{\prime \prime} \otimes\left({ }^{0} T^{\prime \prime}\right) *\right)$-valued holomorphic function

$$
\varphi(t)=\sum \varphi_{h_{1} \ldots h_{q}} t_{1}^{h_{1}} t_{2}^{h_{2}} \ldots t_{q}^{h_{q}}
$$

we set

$$
\|\varphi(t)\|\left\|_{(m)}^{\prime \prime}=\sum ! \mid \varphi_{h_{1} \ldots h_{q}}\right\|_{(m)}^{\prime \prime} t_{1}^{h_{1}} t_{2}^{h_{2}} \ldots t_{q}^{h_{q}}
$$

Then we have

$$
\|\| \psi(t)+\varphi(t)\left\|_{(m)}^{\prime \prime} \ll\right\| \psi(t)\left\|_{(m)}^{\prime \prime}+\right\| \varphi(t) \|_{(m)}^{\prime \prime},
$$

and

$$
\left\|| | \psi ( t ) \varphi ( t ) | i | _ { ( m ) } ^ { \prime \prime } \ll C \left|\left\|\phi(t)|i|_{(m)}^{\prime \prime} \mid\right\| \varphi(t)\|\|_{(m)}^{\prime \prime}\right.\right.
$$

by Sobolev lemma.

Let

$$
A(t)=b / 64 c \sum_{\mu=1}^{\infty} 1 / \mu^{2} c^{\mu}\left(t_{1}+\ldots+t_{q}\right)^{\mu} .
$$

We remark that

$$
A(t)^{\nu} \ll(b / c)^{\nu-1} A(t), \nu=2,3,4, \ldots
$$

For our purpose it suffices to derive the estimates

$$
\left\|\mid \varphi^{\mu}(t)\right\|_{(m)}^{\prime \prime} \ll A(t),
$$

by induction on $\mu$ provided that the constants $b, c$ are chosen properly. For $\mu=1$, this is obvious if $b$ is sufficiently large. Assume therefore that $(5.17)_{\mu-1}$ are established for a $\mu \geq 1$. We have from (5. 12) that

$$
\begin{gathered}
\varphi_{\mu}(t) \underset{\mu+1}{\equiv}-N D_{1}^{*} P\left(\varphi^{\mu-1}(t)\right)+A\left(L N D_{1}^{*} P\left(\varphi^{\mu-1}(t)\right)\right) \\
\underset{\mu+1}{\equiv}-N D_{1}^{*}\left(D_{1} \varphi^{\mu-1}(t)+R_{2}\left(\varphi^{\mu-1}(t)\right)\right) \\
\quad+A\left(L N D_{1}^{*}\left(D_{1} \varphi^{\mu-1}(t)+R_{2}\left(\varphi^{\mu-1}(t)\right)\right)\right) .
\end{gathered}
$$


Then

$$
\left\|\left|\varphi_{\mu}(t)\left\|_{(m)}^{\prime \prime} \ll C_{1}\right\|\right| \varphi^{\mu-1}(t)\right\|_{(m)}^{\prime \prime}+C_{2}\|\| \varphi^{\mu-1}(t) \|_{(m)}^{\prime \prime 2} .
$$

As $\varphi_{\mu}(t)$ is a homogeneous polynomial of degree $\mu$, we have from (5. 18)

$$
\begin{aligned}
\left.\left\|\varphi_{\mu}(t)\right\|\right|_{(m)} ^{\prime \prime} & \ll c_{2}\left|\left\|\varphi^{\mu-1}(t)\right\|\right|_{(m)}^{\prime \prime 2} \\
& \ll c_{2} A(t)^{2} \\
& \ll c_{2} b / c A(t)
\end{aligned}
$$

where the constant $c_{2}$ is independent of $\mu$. Hence if we take $c$ sufficiently large, we have

$$
\|\| \varphi_{\mu}(t) \|_{(m)}^{\prime \prime} \ll A(t),
$$

and so the power series

$$
\varphi(t)=\sum_{\mu=1}^{\infty} \varphi_{\mu}(t)
$$

converges in \|\|$_{(m)}^{\prime \prime}$ norm. Especialy $\varphi(t)$ is in $c^{2}$-class by Sobolev because of $m \geq n+2$.

Q. E. D.

\section{§ 6. Versality}

In this section we shall prove that the deformation constructed in $\S 5$ is a versal family in the sense of M. Kuranishi. Let $Y^{\prime}$ be a complex analytic space of complex dimension $n$ and $Y$ be a relative compact open subset of $Y^{\prime}$ with strongly pseudo-convex smooth boundary $M$. And let $\left(M,{ }^{\circ} T^{\prime \prime}\right)$ be the partially complex structure induced by $Y$. We shall study the complex structure of a neighborhood of M.

Definition 6. 1. Let $N$ be a neighborhood of $M$. Let $S$ be a complex manifold with the origin 0 and $\left\{N_{s} \mid s \in S\right\}$ be a set of complex manifolds depending on $s \in S$. We say that $\left\{N_{s} \mid s \in S\right\}$ forms a complex analytic family of deformations of $N$ if there is a complex manifold $\mathcal{N}$ and a holomorphic map $\varpi$ onto $S$ such that 
(1) $\widetilde{\varpi}^{-1}(0)=N^{\prime} \fallingdotseq N$, and

(2) the rank of the Jacobian $\varpi$ is equal to the complex dimension of $S$ at each point of $N$.

We note that $\widetilde{\varpi}^{-1}(s)$ is a complex submanifold of $\mathscr{N}$.

Proposition 6. 2. Let $\left\{N_{s} \mid s \in S\right\}$ be a complex analytic family of $N$. Then, if we replace $S$ by a smaller neighborhood of 0 if necessary, there is an injective map $F: N \times S \rightarrow$ satisfying the following

(1) $F(y, 0)=i d$ on $N$,

(2) for each $s, F \mid N \times s$ is a diffeomorphism from $N \times s$ to $F(N \times s)$ which is contained in $\varpi^{-1}(s)$,

(3) for each $p \in N, F \mid p \times S$ is a complex analytic map from $p \times S$ to $\mathcal{N}$.

Proof. Let $\left\{\mathscr{U}_{j}^{\prime}\right\}_{, \in A}$ be a finite covering of $\mathscr{N}$ such that on each $\mathscr{U}_{j}^{\prime}$ there is a complex analyitc coordinate $\left(z_{j}^{1}, \ldots, z_{j}^{n}, s\right)$ such that

$$
\mathscr{U}_{j}^{\prime}=\left\{p:\left|z_{j}^{\alpha}(p)\right|<\varepsilon_{j},|s|<\varepsilon\right\}
$$

and these charts are holomorphically related so that

$$
z_{j}^{\alpha}=f_{j \cdot k}^{\alpha}\left(z_{k}, s\right)
$$

on $\mathscr{U}_{j}^{\prime} \cap \mathscr{U}_{k}^{\prime}$ and

$$
\bigcup_{j \in A} \mathscr{U}_{j}^{\prime} \cap \widetilde{\varpi}^{-1}(0) \supset N .
$$

To prove this Proposition 6.2 it suffices to show that there exists a system of $C^{\infty}$ functions $\left\{z_{j}^{\alpha}(s)\right\}_{, \in \Lambda}$ on $\mathscr{U}$, depending holomorphically on $s$ such that

$$
z_{j}^{\alpha}(s)=f_{j: k}^{\alpha}\left(z_{k}(s), s\right)
$$

on $\left(\mathscr{U}_{j} \cap \mathscr{U}_{k}\right) \times S^{\prime}$, where $\left\{\mathscr{U}_{j}\right\}_{j \in \Lambda}$ is a finite covering of $N$ such that $\mathscr{U}_{j} \Subset \mathscr{U}_{j}^{\prime} \cap N, S^{\prime} \Subset S$ and $\mathscr{V}_{j}=\mathscr{U}_{j} \cap M$.

We may assume that $\operatorname{dim} S=1$. We shall construct a system of $C^{\infty}$ funtions $\left\{z_{j}^{\alpha}(s)\right\}_{j \in A}$ formally by induction on the order of the parameter $s$.

We set 


$$
z_{j}^{\alpha \mid 0}(s)=z_{j}^{\alpha}
$$

Then the first step in the inductive proof is completed.

Assume that there exist $C^{\infty}$ functions $\left\{z_{j}^{\alpha \mid \mu}(s)\right\}$ as a polynomial of degree $\mu$ such that

$$
z_{j}^{\alpha \mid \mu}(s) \underset{\bmod s^{\mu+1}}{\equiv} f_{j \cdot k}^{\alpha}\left(z_{k}^{\mu}(s), s\right)
$$

We shall define $C^{\infty}$ functions $z_{j \mid \mu+1}^{\alpha}(s)$ as a homogeneous polynomial of degree $\mu+1$ as follows:

$$
z_{j \mid \mu+1}(s) \underset{\bmod s^{\mu+2}}{\equiv} \sum_{i}\left(\partial f_{j \cdot i} / \partial z_{i}\right) \rho_{i} \sigma_{i \cdot j}
$$

where $z_{j \cdot \mu+1}(s)=\left(z_{j \mid \mu+1}^{\alpha}(s)\right)_{1 \leq \alpha \leq n}, \quad \sigma_{i \cdot j}(s)=\left(\sigma_{i \cdot j}^{\alpha}(s)\right)_{1 \leq \alpha \leq n}, \sigma_{i \cdot j}^{\alpha}(s)=z_{i}^{\alpha \mid \mu}(s)-$ $f_{i \cdot j}^{\alpha}\left(z_{j}^{\mu}(s), s\right),\left(\partial f_{j \cdot i} / \partial z_{i}\right)$ denotes the matrix $\left(\partial f_{i \cdot j}^{\alpha} / \partial z_{j}^{\beta}\right)_{1 \leq \alpha, \beta \leq n},\left\{\rho_{i}\right\}_{i \in A}$ denotes a partion of unity for the open covering $\left\{\mathscr{U}_{i}^{\prime}\right\}_{i \in \Lambda}$, and

$$
z_{j \mid \mu+1}(s)=\left(z_{j \mid \mu+1}^{\alpha}(s)\right)_{1 \leq \alpha \leq n} .
$$

From the assumption (6.1) ${ }_{\mu}$, we have

$$
\sigma_{i \cdot j}(s) \underset{\bmod s^{\mu+1}}{\equiv} 0 .
$$

From the relations :

$$
\begin{aligned}
& \sigma_{i \cdot l}^{\alpha}(s)=z_{i}^{\alpha \mid \mu}(s)-f_{i \cdot l}^{\alpha}\left(z_{l}^{\mu}(s), s\right), \\
& \sigma_{i \cdot j}^{\alpha}(s)=z_{i}^{\alpha \mid \mu}(s)-f_{i \cdot j}^{\alpha}\left(z_{j}^{\mu}(s), s\right),
\end{aligned}
$$

and

$$
\sigma_{j \cdot l}^{\alpha}(s)=z_{j}^{\alpha \mid \mu}(s)-f_{j \cdot l}^{\alpha}\left(z_{l}^{\mu}(s), s\right) .
$$

So we have

$$
\begin{aligned}
\sigma_{i \cdot j}^{\alpha}(s) & =z_{i}^{\alpha \mid \mu}(s)-f_{i \cdot j}^{\alpha}\left(z_{j}^{\mu}(s), s\right) \\
= & z_{i}^{\alpha \mid \mu}(s)-f_{i \cdot j}^{\alpha}\left(\sigma_{j \cdot l}(s)+f_{j \cdot l}\left(z_{l}^{\mu}(s), s\right)\right. \\
\underset{\bmod s}{\equiv}-\sum_{\beta} f_{i \cdot j}^{\alpha} / \partial z_{j}^{\beta} \sigma_{j \cdot l}^{\beta}(s) & \quad+z_{i}^{\alpha \mid \mu}(s)-f_{i \cdot j}^{\alpha}\left(f_{j \cdot l}\left(z_{l}^{\mu}(s), s\right), s\right) .
\end{aligned}
$$

Therefore

$$
\sigma_{i \cdot \rho}(s)+\left(\partial f_{i \cdot j} / \partial z_{j}\right) \sigma_{j \cdot l}(s) \underset{\bmod s^{\mu+2}}{\equiv} \sigma_{i \cdot l}(s) .
$$

From the definition of $z_{\jmath \mu+1}^{\alpha}(s)$, we have 


$$
-\sigma_{l \cdot j}(s) \underset{\bmod s^{\mu+2}}{\equiv} z_{l \mid \mu+1}(s)-\partial f_{l \cdot j} / \partial z_{j} z_{j \mid \mu+1}(s)
$$

Therefore we have

$$
\begin{aligned}
& z_{i}^{\alpha \mid \mu}(s)+z_{i \mid \mu+1}^{\alpha}(s) \\
& \underset{\bmod s^{\mu+2}}{\equiv} f_{i \cdot j}^{\alpha}\left(z_{j}^{\mu}(s)+z_{j \mid \mu+1}(s), s\right) .
\end{aligned}
$$

This finishes the construction of the formal power series

$$
z_{j}^{\alpha}(s)=z_{j}^{\alpha}+z_{j \mid 1}^{\alpha} s+\ldots,
$$

such that

$$
z_{i}^{\alpha}(s)=f_{i \cdot j}^{\alpha}\left(z_{j}(s), s\right)
$$

as formal power series.

We shall prove that power series $z_{i}^{\alpha}(s)$ converges for $|s|<\delta$ for some small $\delta>0$. We dominate $z_{i}^{\alpha}(s)$ with a convergent series. We fix some notation. Let $\phi\left(z_{i}, s\right)=\sum_{m=0}^{\infty} \psi_{m}\left(z_{i}\right) s^{m}$ be a power series where $\psi_{m}\left(z_{i}\right)=\left(\phi_{m}^{\prime}\left(z_{i}\right), \ldots, \phi_{m}^{n}\left(z_{i}\right)\right), z_{i} \in \mathscr{U}_{i}$. Let $a(s)=\sum_{m=0}^{\infty} a_{m} s^{m}, a_{m} \geq 0$ be a series with real, positive coefficients. We write $\phi\left(z_{i}, s\right) \ll a(s)$ if $\left|\psi_{m}^{\alpha}\left(z_{i}\right)\right|$ $\leq a_{m}$ for all $z_{i} \in \mathscr{U}_{i}$ and all $\alpha=1, \ldots, n$. The norm of $\phi_{m}$ is the Hölder $k$-norm. We shall recall the power series

$$
A(s)=b / 16 c \sum_{m=1}^{\infty}(c s)^{m} / m^{2} .
$$

It suffices to prove

$$
z_{i}(s)-z_{i} \ll A(s)
$$

That is to say

$$
z_{1}^{\alpha \mid \mu}(s)-z_{i} \ll A(s)
$$

for $\mu=1,2,3, \ldots$.

Let us prove $(6.4)_{1}$ : We want to show that

$$
z_{i}^{\alpha}(s) \ll A(s)=b / 16\{s+\ldots\} .
$$

From $\mathscr{U}_{j} \Subset \mathscr{U}^{\prime} \cap N$, this is satisfied for $b$ large enough.

By induction, assume (6.4) ${ }_{\mu}$ and let us prove $(6.4)_{\mu+1}$. Remember

$$
\begin{aligned}
& z_{i}^{\alpha \mid \mu+1}(s)=z_{i}^{\alpha \mid \mu}(s)+z_{i \mid \mu+1}^{a}(s), \\
& \sigma_{i \cdot j}^{\alpha}(s)=z_{i}^{\alpha \mid \mu}(s)-f_{i \cdot j}^{\alpha}\left(z_{j}^{\mu}(s), s\right),
\end{aligned}
$$


and

$$
z_{j \mid \mu+1}(s) \underset{\bmod s^{\mu+2}}{\equiv} \sum_{i}\left(\partial f_{j \cdot i} / \partial z_{i}\right) \rho_{i} \sigma_{i \cdot j \cdot}
$$

Remember the definitions of $\mathscr{V}_{j}$ and $\mathscr{U}_{j}^{\prime}$. Then $f_{i \cdot j}\left(z_{j}, s\right)$ is defined on $\mathscr{U}_{i}^{\prime} \cap \mathscr{U}_{j}^{\prime}$ and $f_{i \cdot j}\left(z_{j}+y, s\right)$ is holomorphic for $\left(z_{j}, s\right) \in \mathscr{V}_{j}$. So there exists a $K>0$ such that

$$
\left|f_{i j}^{(m, n)}\left(z_{j}\right)\right|<K^{m+n},
$$

where $f_{i \cdot j}\left(z_{j}+y, s\right)=\sum_{m, n \geq 0} f_{i \cdot j}^{(m, n)}\left(z_{j}\right) y^{m} s^{n}$. We want to estimate the homogeneous term of $\partial f_{j i} / \partial z_{i} \rho_{i} \sigma_{i \cdot j}$ of order $\mu+1$

$$
\begin{aligned}
{\left[\partial f_{j \cdot i} / \partial z_{i} \rho_{i} \sigma_{i \cdot j}^{\alpha}(s)\right]_{\mu+1} } & =\left[\partial f_{j \cdot i} / \partial z_{i} \rho_{i}\left(z_{i}^{\alpha \mid \mu}(s)-f_{i \cdot j}^{\alpha}\left(z_{j \mid \mu+1}(s)\right)\right]_{\mu+1}\right. \\
& \ll c^{\prime} \sum_{m+n \geq 2}^{\infty} K^{m+n} A(s)^{m+n} \\
& \ll c^{\prime} K^{2} \sum_{m+n \geq 0}^{\infty}\left(\frac{b}{c} K\right)^{m+n} \frac{b}{c} A(s),
\end{aligned}
$$

because of the induction assumption $(6.4)_{\mu}$. If we choose $c$ so large, then

$$
\left|z_{i \mid \mu+1}(s)\right|<A(s) .
$$

Therefore we see that the power series $z_{i}^{\alpha}(s)$ converges for $|s|<\delta_{k}$ for some small $\delta_{k}>0$. We may assume that the functions $z_{i}^{\alpha}(s)$ are $m$-times continuously differentiable if we put $k \geq m$. From now on we fix these functions $z_{i}^{\alpha}(s),|s|<\delta_{k}$.

Lemma 6. 3. Let $\left\{N_{s} \mid s \in S\right\}$ be a complex analytic family of $N$. Then there exists $\omega(s) \in \Gamma_{(m)}^{\prime \prime}\left(N, T^{\prime} N \otimes\left(T^{\prime \prime} N\right)^{*}\right)$ depending analytically on s such that

$$
\left(X^{\prime}+\omega(s)\left(X^{\prime}\right)\right) z_{j}^{\alpha}(s)=0
$$

on $\mathscr{U}_{j}$, for any $X^{\prime} \in T^{\prime \prime} N$.

Proof. Let $\left\{z_{j}^{\alpha}\right\}_{1 \leq \alpha \leq n}$ be a complex coordinate system. Using this coordinate we define $C^{\infty}$ functions $(\omega(s))_{\beta \cdot \alpha}^{j}$ as follows.

$$
\omega(s)\left(\partial / \partial \bar{z}_{j}^{\alpha}\right)=\sum_{\beta}(\omega(s))_{\beta \cdot \alpha}^{j} \partial / \partial z_{j}^{\beta} .
$$

Using these funtions we rewrite (6.5) as follows. 


$$
\partial z_{j}^{\alpha}(s) / \partial \bar{z}_{j}^{r}+\sum_{\beta}(\omega(s))_{\beta \cdot \tau}^{j} \partial z_{j}^{\alpha}(s) / \partial z_{j}^{\beta}=0 .
$$

We note that the relations

$$
\partial z_{j}^{\alpha} / \partial z_{j}^{\beta}=\delta_{\beta \cdot \alpha}
$$

hold. Therefore the matrix $\left(\partial z_{j}^{\alpha}(s) / \partial z_{j}^{\beta}\right)_{1 \leq \alpha, \beta \leq n}$ has an inverse if $s$ is sufficiently small. Since $\left(\omega(s)_{\beta}^{j}\right.$.r is a solution of the linear equation (6.6), it depends analytically on $s$. So we have our lemma.

Q.E. D.

After this we shall use this notation $\left(N,{ }^{\omega(s)} T^{\prime \prime}\right)$ instead of a complex analytic family $\left\{N_{s} \mid s \in S\right\}$ of $N$. We shall call $\left(N,{ }^{\infty(s)} T^{\prime \prime}\right)$ a deformation of $N$.

Let $T$ be a complex manifold with the origin 0 .

Definition 6. 4. Let $\left\{\left(M,{ }^{\varphi(t)} T^{\prime \prime}\right) \mid t \in T\right\}$ be a set of partially complex structures depending on $t \in T$. We call a set of partially complex structures $\left\{\left(M,{ }^{\varphi(t)} T^{\prime \prime}\right) \mid t \in T\right\}$ a family if $\varphi(t)$ satisfies the following: $\varphi(t)$ depends analytically on $t$ as a map $T$ to the Banach-manifold $\Gamma_{(m)}^{\prime \prime}\left(M, T^{\prime} \otimes\left({ }^{0} T^{\prime \prime}\right)^{*}\right)$, and $\varphi(0)=0$.

Under these notations, we shall define a versal family of partially complex structures $\left\{\left(M,{ }^{\varphi(t)} T^{\prime \prime}\right) \mid t \in T\right\}$.

Definition 6. 5. We say that a family of partially complex structures $\left\{\left(M,{ }^{(t)} T^{\prime \prime}\right) \mid t \in T\right\}$ is versal if the family satisfies the following condition.

For any neighborhood $N$ of $M$ and any complex analytic family $\left\{N_{s} \mid s \in S\right\}$ of deformations $N$, there exist differentiable embeddings $f_{5(s)}$ from $M$ to $N$ and an analytic map $h$ from $S$ to $T$ such that

$$
h(0)=0
$$

and

$$
\omega(s) \cdot f_{\zeta(s)}=\varphi(h(s))
$$

for any $s$ close to the origin 0 in $S$, where $\omega(s)$ denotes the elements in $\Gamma_{(m)}^{\prime \prime}\left(M, T^{\prime} \otimes\left({ }^{0} T^{\prime \prime}\right)^{*}\right)$ defined by the complex analytic family in Lemma 6.3 , and $\omega(s) \cdot f_{5(s)}$ denotes the partially complex structure on 
$M$ induced via $f_{\zeta(s)}$ by $\left(N,{ }^{\omega(s)} T^{\prime \prime}\right)$.

Theorem 6. 6. The deformation constructed in $\S 5$ is versal.

Proof. We shall prove this theorem using the above $C^{\infty}$ functions $\left\{z_{j}^{\alpha}(s)\right\}_{j \in \Lambda}$ and a prior estimates obtained in $\S 3$. It makes no difference for the proof and it makes the writing much easier to assume $\operatorname{dim} S=1$.

For any deformations of complex structures $\left(N,{ }^{\omega(s)} T^{\prime \prime}\right)$, there exist a finite covering $\left\{\mathscr{U}_{j}\right\}_{j \in \Lambda}$ of $N$ and differentiable isomorphism $z_{j}(s)=$ $\left(z_{j}^{1}(s), \ldots, z_{j}^{n}(s)\right)$ from $\mathscr{U}_{j}$ to a ball depending analytically on $s$ such that

$$
z_{j}^{\alpha}(s)=f_{j \cdot k}^{\alpha}\left(z_{k}(s), s\right) \text { on } \mathscr{U}_{j} \cap \mathscr{U}_{k}
$$

and

$$
z_{j}^{\alpha}(0)=z_{j}^{\alpha},
$$

where $f_{j \cdot k}^{\alpha}$ is holomorphic in $z_{k}^{\alpha}(s)$ and $s,\left\{z_{j}^{\alpha}\right\}$ is a complex coordinate system of $\left(N,{ }^{\omega(0)} T^{\prime \prime}\right)$ and $\left\{z_{j}^{\alpha}(s)\right\}$ a complex coordinate system of $\left(N,{ }^{\infty(s)} T^{\prime \prime}\right)$. After this we identify a ball in $C^{n}$ with $\mathscr{U}_{j} \times s$ via the diffeomorphism $z_{j}(s)$ if there is no confusion. We define embeddings $f_{\xi^{(1)}(s)}$ from $\mathscr{W}_{j} \cap M \times\{s:|s|<\varepsilon\}$ to $\mathscr{U}_{j} \times\{s:|s|<\varepsilon\}$, where $\mathscr{W}_{j} \Subset \mathscr{U}_{j}$ and $\cup_{j \in \Lambda} \mathscr{W}_{j} \supset M$ as follows: For each $\left(z_{j}(s), s\right) \in \mathscr{W}_{j} \cap M \times\{s:|s|<\varepsilon\}$, we set

$$
\begin{aligned}
& f_{\xi^{(1)}(s)}\left(z_{j}(s), s\right) \\
& =\left(z_{j}^{\alpha}(s)+\zeta^{(1)}\left(z_{j}^{\alpha}(s)\right) s, s\right) \\
& \in \mathscr{U}_{j} \times\{s:|s|<\varepsilon\},
\end{aligned}
$$

where $\zeta^{(1)}$ is any element of $\Gamma\left(M, T^{\prime}\right)$. This map is well defined if $\varepsilon$ is sufficiently small. This is not defined globally on $M \times\{s:|s|<\varepsilon\}$ but is well defined modulo $s^{2}$. In fact the following relation assures this.

$$
\begin{aligned}
& z_{j}^{\alpha}(s)+\zeta^{(1)}\left(z_{j}^{\alpha}(s)\right) s \\
& \underset{\bmod s^{2}}{\equiv} f_{j \cdot k}^{\alpha}\left(z_{k}(s)+\zeta^{(1)}\left(z_{k}(s), s\right), s\right)
\end{aligned}
$$

on

$$
\mathscr{U}_{j} \cap \mathscr{U}_{k} \cap M
$$


From this, we shall define $\omega(s) \circ f_{\xi^{(1)}(s)}$ in $\Gamma\left(\mathscr{U}_{j} \cap M, T^{\prime} \otimes\left({ }^{0} T^{\prime \prime}\right)^{*}\right)$ as follows :

$$
\left(X+\omega(s) \circ f_{\zeta^{(1)}(s)}(X)\right)\left(z_{j}^{\alpha}(s)+\zeta^{(1)}\left(z_{j}^{\alpha}(s)\right) s\right)=0
$$

on $\mathscr{U}_{j} \cap M, \alpha-1,2, \ldots, n$ and $X \in{ }^{\circ} T^{\prime \prime}$.

Then we have the following lemma.

Lemma 6. 7. $\omega(s) \circ f_{\xi_{(s)}^{(1)}}$ depends holomorphically on $s$.

Proof. Let $h$ be a defining function for $M$ in $\mathscr{U}_{j}$ and $\left\{z_{j}^{\alpha}\right\}_{1 \leq \alpha \leq n}$ be a complex coordinate system. Then there exists a system of moving frame $\left\{e_{j}^{\beta}\right\}_{\beta=1,2, \ldots, n-1}$ of $\left.{ }^{0} T^{\prime \prime}\right|_{\mathscr{U}_{j} \cap M}$ and moving frame $\left\{\bar{e}_{j}^{\beta}\right\}_{\beta=1,2, \ldots, n-1}$, $\zeta$ of $\left.T^{\prime}\right|_{\mathscr{U}_{j} \cap M}$ such that

$$
e_{j}^{\beta}+\partial / \partial \bar{z}_{j}^{\beta}=h_{\beta} / h_{\bar{n}} \partial / \partial \bar{z}_{j}^{n},
$$

and

$$
\bar{e}_{j}^{n}=\partial / \partial z_{j}^{n}-h_{n} / h_{\bar{n}} \partial / \partial \bar{z}_{j}^{n},
$$

where we assume that $\partial h / \partial z_{j}^{n} \neq 0$ on $\mathscr{U}_{j} \cap M$. Using this frame we define $C^{\infty}$ functions $\left(\omega(s) \circ f_{\xi_{(1)}^{(s)}}\right)_{r, \beta}^{j}$ as follows.

$$
\begin{aligned}
\omega(s) \circ f_{\zeta^{(1)}(s)}\left(e_{j}^{\beta}\right)= & \sum_{r=1}^{n-1}\left(\omega(s) \circ f_{\xi^{(1)}(s)}\right)_{r, \beta}^{j} \bar{e}_{j}^{r} \\
& +\left(\omega(s) \circ f_{\zeta^{(1)}(s)}\right)_{n, \beta}^{j} \bar{e}_{j}^{n} .
\end{aligned}
$$

Using these functions we rewrite $(6.6)^{\prime}$ as follows : for any $e_{j}^{\beta} \beta=$ $1,2, \ldots, n-1$, we have

$$
\begin{aligned}
& \sum_{r=1}^{n-1}\left(\omega(s) \circ f_{\zeta^{(1)}(s)}\right)_{r, \beta}^{j} \bar{e}_{j}^{r}\left(z_{j}^{\alpha}(s)+\zeta^{(1)}\left(z_{j}^{\alpha}(s)\right) s\right) \\
& +\left(\omega(s) \circ f_{\zeta^{(1)}(s)}\right)_{n, \beta}^{\prime} \bar{e}_{j}^{n}\left(z_{j}^{\alpha}(s)+\zeta^{(1)}\left(z_{j}^{\alpha}(s)\right) s\right) \\
& +e_{j}^{\beta}\left(z_{j}^{\alpha}(s)+\zeta^{(1)}\left(z_{j}^{\alpha}(s)\right) s\right)=0 .
\end{aligned}
$$

We note that the relations

$$
\bar{e}_{j}^{r} \cdot z_{j}^{\alpha}=\delta_{r \cdot \alpha} \quad 1 \leq \alpha, r \leq n
$$

holds.

We put $(n, n)$-matrix $B(s)$ as follows.

$$
B(s)=\left(\bar{e}_{j}^{r} \cdot\left(z_{j}^{\alpha}(s)+\zeta^{(1)}\left(z_{j}^{\alpha}(s)\right) s\right)\right)_{1 \leq \alpha, r \leq n}
$$


Of course $B(0)=E_{n}$ and $B(s)$ depends analytically on $s$. If $s$ is sufficiently small, the matrix $B(s)$ has the inverse. Since $\left\{\omega(s) f_{\xi^{(1)}(s)}\right\}_{r, \beta \beta=1,2, \ldots, n}^{j}$ is a solution of the linear equation (6.7) with analytic functions in $s$ as coefficients, we have our lemma.

Q. E. D.

By this lemma we can expand the left hand sides of (6.6) into a power series of $s$. Then we have

$$
X\left(z_{j}^{\alpha}(s)\right)+\omega(s) \circ f_{\xi^{(1)}(s)}(X)\left(z_{j}^{\alpha}(s)\right)+X\left(\zeta^{(1)}\left(z_{j}^{\alpha}(s)\right) s\right) \underset{\bmod s^{2}}{\equiv 0}
$$

for any $\zeta^{(1)} \in \Gamma\left(M, T^{\prime}\right)$ and any $X \in \Gamma\left(M,{ }^{\circ} T^{\prime \prime}\right)$. In (6.8) putting $\zeta^{(1)}=0$, we have

$$
X\left(z_{j}^{\alpha}(s)\right)+\omega(s) \circ f_{0}(X)\left(z_{j}^{\alpha}(s)\right) \underset{\bmod s^{2}}{\equiv 0 .}
$$

From (6.8) and (6.9), we have

$$
\begin{aligned}
& \omega(s) \circ f_{\xi^{(1)}(s)}(X)\left(z_{j}^{\alpha}(s)\right) \underset{\bmod ^{2}}{\equiv}-X\left(\zeta^{(1)}\left(z_{j}^{\alpha}(s)\right)\right) s \\
&+\omega(s) \circ f_{0}(X)\left(z_{j}^{\alpha}(s)\right) \\
& \equiv-X\left(\zeta^{(1)}\left(z_{j}^{\alpha}\right) s\right)+\omega(s) \circ f_{0}(X)\left(z_{j}^{\alpha}\right) .
\end{aligned}
$$

So we have

$$
\omega(s) \circ f_{\zeta^{(1)}(s)}(X) \underset{\bmod s^{2}}{\equiv}-\bar{\partial}_{T^{\prime}} \zeta^{(1)} s(X)+\omega(s) \circ f_{0}(X) .
$$

For any power series $h(s)=\sum_{m=1}^{\infty} h_{m} s^{m}$, we put

$$
h^{(k)}(s)=\sum_{m=1}^{k} h_{m} s^{m},
$$

where $h_{m}=\left(h_{m}^{(1)}, h_{m}^{(2)}, \ldots, h_{m}^{(q)}\right), q=\operatorname{dim} \mathscr{H}$. Let $\left\{\beta_{\lambda}\right\}_{1 \leq \lambda \leq q}$ be a base of $\mathscr{H}$. Then from the construction of $\varphi(t)$, we have

$$
\left.\varphi(h(s))=\sum_{i=1}^{q} h_{1}^{(\lambda)} \beta_{\lambda} s+\mathcal{O} \text { (higher order of } s\right) .
$$

After the above preparation, we shall find $h(s)$ and the map $f_{\varepsilon(s)}$ such that

$$
\omega(s) \circ f_{\zeta(s)} \equiv \underset{\bmod s^{2}}{\equiv} \varphi(h(s)) .
$$

This equation becomes 


$$
\omega(s) \circ f_{0}-\bar{\partial}_{T} \zeta^{(1)} s \underset{\bmod s^{2}}{\equiv} \sum_{\lambda=1}^{q} h_{1}^{(\lambda)} \beta_{\lambda} s .
$$

This equation has solutions $h_{1}^{(1)}, \zeta^{(1)}$. In fact from the relation

$$
\bar{\partial}_{T^{\prime}}^{(1)} \omega(s) \circ f_{0}+R_{2}\left(\omega(s) \circ f_{0}\right)+R_{3}\left(\omega(s) \circ f_{0}\right)=0
$$

we have

$$
\bar{\partial}_{T^{\prime}}^{(1)} \omega(s) \circ f_{0} \equiv 0 .
$$

Therefore we define homogeneous polynomials $h_{1}(s)$ and $\zeta^{(1)}(s)$ of degree 1 as follows:

$$
h_{1}(s) \underset{\bmod s^{2}}{\equiv} \mathscr{L}\left(H_{T^{\prime}}^{(1)}\left(\omega(s) \circ f_{0}\right)\right)
$$

and

$$
\zeta^{(1)}(s) \underset{\bmod s^{2}}{\equiv} \bar{\partial}_{T^{\prime}}^{*} N_{T^{\prime}}\left(-\mathscr{L}\left(H_{T^{\prime}}^{(1)}\left(\omega(s) \circ f_{0}\right)\right)+\omega(s) \circ f_{0}\right) .
$$

Then (6.12) is satisfied.

We shall prove that we can find solutions $h(s)$ and $f_{5(s)}$ formally by induction on the order of powers in the parameter $s$

$$
\omega(s) \circ f_{\zeta(s)}=\varphi(h(s)) .
$$

The first step in the inductive proof is completed.

Assume that the embedding maps $f_{\xi^{(k)}(s)}$ from $M \cap \mathscr{W}_{j} \times\left\{s:|s|<\varepsilon_{k}\right\}$ to $\mathscr{U}_{j} \times\left\{s:|s|<\varepsilon_{k}\right\}$ are determined so that $f_{\xi^{(k)}(s)}$ is well defined globally modulo $s^{k+1}$ on $M$ and the analytic maps $h^{(k)}(s)$ from $S$ to $T$ is determined as a polynomial of degree $k$ satisfying the following:

$$
\omega(s) \circ f_{\xi^{(k)}(s)} \underset{\bmod s^{k+1}}{\equiv} \varphi\left(h^{(k)}(s)\right) .
$$

Lemma 6. 8. Assuming these situation, the map $f_{\xi^{(k)}(s)}$ can be extended to a map $f_{\xi^{(k)}(s)+\eta_{k+1}^{j}(s)}$, which is well defined globally modulo $s^{k+2}$ on $M$.

Proof. We shall define embedding maps $f_{\xi^{(k)}(s)+\eta_{k+1}^{j}(s)}$ from $\left(M \cap \mathscr{W}_{j}\right) \times\left\{s:|s|<\varepsilon_{k+1}\right\}$ to $\mathscr{U}_{j} \times\left\{s:|s|<\varepsilon_{k+1}\right\}$ as follows: First we define an element $\eta_{k+1}^{j}(s)$ in $C^{0}\left(\left\{\mathscr{W}_{j} \cap M\right\}, T^{\prime} N\right)$ as a homogeneous polynomial of degree $k+1$ as follows: 


$$
\eta_{k+1}^{j}(s) \underset{\bmod s^{k+2}}{\equiv} \sum_{i}\left(\partial f_{j i} / \partial z_{i}\right) \rho_{i} \sigma_{i \cdot j}
$$

where $\sigma_{i \cdot j}=\left(\sigma_{i \cdot j}^{\alpha}\right) \quad 1 \leq \alpha \leq m$,

$$
\sigma_{i, j}^{\alpha}=z_{\alpha}^{i}(s)+\zeta_{i}^{(k) \cdot \alpha}(s)-f_{i \cdot j}^{\alpha}\left(z_{j}(s)+\zeta_{j}^{(k)}(s), s\right),
$$

$\left(\partial f_{j i} / \partial z_{i}\right)$ denotes the $(n, n)$-matrix $\left(\partial f_{j \cdot i}^{\alpha} / \partial z_{i}^{\beta}\right)_{1 \leq \alpha, \beta \leq n}$ and $\eta_{k+1}^{j}(s)=$ $\left(\eta_{k+1}^{j \cdot \alpha}(s)\right)_{1 \leq \alpha \leq n}$. Then from the assumption, we have

$$
\sigma_{i \cdot j} \equiv 0 \bmod s^{k+1} \text {. }
$$

From the relations

$$
\begin{aligned}
& \sigma_{i, l}^{\alpha}=z_{i}^{\alpha}(s)+\zeta_{i}^{(k), \alpha}(s)-f_{i, l}^{\alpha}\left(z_{l}(s)+\zeta_{l}^{(k)}(s), s\right), \\
& \sigma_{i, j}^{\alpha}=z_{i}^{\alpha}(s)+\zeta_{i}^{(k), \alpha}(s)-f_{i, j}^{\alpha}\left(z_{j}(s)+\zeta_{j}^{(k)}(s), s\right)
\end{aligned}
$$

and

$$
\sigma_{j, l}^{\alpha}=z_{j}^{\alpha}(s)+\zeta_{j}^{(k), \alpha}(s)-f_{j, l}^{\alpha}\left(z_{l}(s)+\zeta_{l}^{(k)}(s), s\right),
$$

we have

$$
\begin{aligned}
\sigma_{i, j}^{\alpha} & =z_{i}^{\alpha}(s)+\zeta_{i}^{(k), \alpha}(s)-f_{i, j}^{\alpha}\left(\sigma_{j, l}+f_{j, l}\left(z_{l}(s)+\zeta_{l}^{(k)}(s), s\right), s\right) \\
& \equiv-\sum_{\beta} \partial f_{i, j}^{\alpha} / \partial z_{j}^{\beta} \sigma_{j, l}^{\beta}+z_{i}^{\alpha}(s)+\zeta_{i}^{(k), \alpha}(s) \\
& \equiv \bmod ^{k+2} \\
& -f_{i, j}^{\alpha}\left(f_{j, l}\left(z_{l}(s)+\zeta_{l}^{(k)}(s), s\right), s\right) .
\end{aligned}
$$

So

$$
\sigma_{i, j}+\left(\partial f_{i, j} / \partial z_{j}\right) \sigma_{j, l} \underset{\bmod s^{k+2}}{\equiv} \sigma_{i, l}
$$

and from the definition of $\eta_{k+1}^{j}(s)$, we have

$$
-\sigma_{l, j}=\eta_{k+1}^{l}(s)-\left(\partial f_{l, j} / \partial z_{j}\right) \eta_{k+1}^{j}(s) .
$$

Therefore we have

$$
z_{i}^{\alpha}(s)+\zeta_{i}^{(k), \alpha}(s)+\eta_{k+1}^{i, \alpha}(s) \underset{\bmod s^{k+2}}{\equiv} f_{i, j}^{\alpha}\left(z_{j}(s)+\zeta_{j}^{(k)}(s)+\eta_{k+1}^{j}(s), s\right) .
$$

For any $\Gamma\left(M, T^{\prime}\right)$-valued homogeneous polynomial $\zeta_{k+1}(s)$ of degree $k+1$, we have

$$
\begin{aligned}
& z_{i}^{\alpha}(s)+\zeta_{i}^{(k), \alpha}(s)+\eta_{k+1}^{i, \alpha}(s)+\zeta_{k+1}(s) z_{i}^{\alpha}(s) \\
& \quad \equiv \underset{\text { mod } s^{k+2}}{\equiv} f_{i, j}^{\alpha}\left(z_{j}(s)+\zeta_{j}^{(k)}(s)+\eta_{k+1}^{j}(s)+\zeta_{k+1}(s)\left(z_{j}^{\alpha}(s)\right), s\right) .
\end{aligned}
$$

Now we shall solve the following equation. We shall find solutions $\zeta_{k+1}(s)$ and $h_{k+1}(s)$ as follows. 


$$
\omega(s) \circ f_{\xi^{(k)}(s)+\eta_{k+1}^{j}(s)+\varepsilon_{k+1}(s)} \equiv \underset{\bmod s^{k+2}}{\equiv} \varphi\left(h^{(k)}(s)+h_{k+1}(s)\right) .
$$

The left hand side of this differential equation is well defined modulo $s^{k+2}$ from (6.13).

By definition of $\omega(s) \circ f_{\xi^{(k)}(s)+\eta_{k+1}^{j}(s)+\zeta_{k+1}(s)}$, we have

$$
\begin{aligned}
\left(X+\omega(s) \circ f_{\zeta^{(k)}(s)+\eta_{k+1}^{j}(s)+\zeta_{k+1}(s)}(X)\right)\left(z_{j}^{\alpha}(s)+\right. \\
\left.\zeta_{j}^{(k)}(s)+\eta_{k+1}^{j}(s)+\zeta_{k+1}(s)\left(z_{j}^{\alpha}(s)\right)\right)=0,
\end{aligned}
$$

$\alpha=1,2, \ldots, n$ for any $X \in \Gamma\left(M,{ }^{\circ} T^{\prime \prime}\right)$.

Then we can prove the following lemma using the method of the proof of Lemma 6.7.

Lemma 6. 9. For any $\Gamma\left(M, T^{\prime}\right)$-valued homogeneous polynomial $\zeta_{k+1}(s)$ with degree $k+1$ in $s, \omega(s) \circ f_{\xi^{(k)}(s)+\eta_{k+1}^{j}(s)+\zeta_{k+1}(s)}$ depends analytically on $s$.

We can expand both hand sides of (6.14) into power series of $s$. Then we have

$$
\begin{aligned}
& X z_{j}^{\alpha}(s)+\omega(s) \circ f_{\zeta^{(k)}(s)+\eta_{k+1}^{j}(s)+\zeta_{k+1}(s)}(X) z_{j}^{\alpha}(s) \\
& +X \zeta_{j}^{(k), \alpha}(s)+\omega(s) \circ f_{\zeta^{(k)}(s)+\eta_{k+1}^{j}(s)+\zeta_{k+1}(s)}(X) \zeta_{j}^{(k), \alpha}(s) \\
& +X \eta_{k+1}^{j, \alpha}(s)+\omega(s) \circ f_{\zeta^{(k)}(s)+\eta_{k+1}^{j}(s)+\zeta_{k+1}(s)} \eta_{k+1}^{j, \alpha}(s) \\
& +X \zeta_{k+1}\left(z_{j}^{\alpha}(s)\right)+\omega(s) \circ f_{\zeta^{(k)}(s)+\eta_{k+1}^{j}(s)+\zeta_{k+1}(s)} \zeta_{k+1}(s)\left(z_{j}^{\alpha}(s)\right) \\
& \equiv 0 \\
& \equiv \bmod ^{k+2}
\end{aligned}
$$

Putting $\zeta_{k+1}(s)=0$ in (6.15), we have

$$
\begin{aligned}
& X z_{j}^{\alpha}(s)+\omega(s) \circ f_{\zeta^{(k)}(s)+\eta_{k+1}^{j}(s)}(X) z_{j}^{\alpha}(s) \\
& +X \zeta_{j}^{(k), a}(s)+\omega(s) \circ f_{\zeta^{(k)}(s)+\eta_{k+1}^{j}(s)}(X) \zeta_{j}^{(k), \alpha}(s) \\
& +X \eta_{k+1}^{j, \alpha}(s)+\omega(s) \circ f_{\zeta^{(k)}(s)+\eta_{k+1}^{j}(s)}(X) \eta_{k+1}^{j, \alpha}(s) \\
& \underset{\bmod s^{k+2}}{\equiv} 0 .
\end{aligned}
$$

From (6.15) and (6.16), we have 
(6. 17)

$$
\begin{aligned}
& \left(\omega(s) \circ f_{\xi^{(k)}(s)+\eta_{k+1}^{j}(s)+\zeta_{k+1}(s)}(X)-\omega(s) \circ f_{\xi^{(k)}(s)+\eta_{k+1}^{j}(s)}(X)\right) z_{j}^{\alpha}(s) \\
& +X \zeta_{k+1}(s)\left(z_{j}^{\alpha}(s)\right) \underset{\text { mod } s^{k+2}}{\equiv} 0 .
\end{aligned}
$$

Therefore we have

$$
\text { (6.18) } \begin{aligned}
& \left(\omega(s) \circ f_{\xi^{(k)}(s)+\eta_{k+1}^{j}(s)+\zeta_{k+1}(s)}(X)-\omega(s) \circ f_{\xi^{(k)}(s)+\eta_{k+1}^{j}(s)}(X)\right) z_{j}^{\alpha} \\
& +X \zeta_{k+1}(s) z_{j=0}^{\alpha} \underset{\text { mod } s^{k+2}}{\equiv} 0,
\end{aligned}
$$

that is to say,

$$
\begin{aligned}
& \omega(s) \circ f_{\xi^{(k)}(s)+\eta_{k+1}^{j}+(s)+\zeta_{k+1}(s)} \\
& \underset{\text { mod s } s^{k+2}}{\equiv} \omega(s) \circ f_{\xi^{(k)}(s)+\eta_{k+1}^{j}(s)}-\bar{\partial}_{T^{\prime}} \zeta_{k+1}(s) .
\end{aligned}
$$

Now we shall prove that the following equation has solutions $\zeta_{k+1}(s)$ and $h_{k+1}(s)$ :

$$
\omega(s) \circ f_{\xi^{(k)}(s)+\eta_{k+1}^{j}(s)+\varepsilon_{k+1}(s)} \underset{\bmod s^{k+2}}{\equiv} \varphi\left(h^{(k)}(s)+h_{k+1}(s)\right) .
$$

From (6.19), we have

$$
\begin{array}{r}
-\bar{\partial}_{T^{\prime}} \zeta_{k+1}(s)+\omega(s) \circ f_{\xi^{(k)}(s)+\eta_{k+1}^{j}(s)} \\
\underset{\substack{\bmod s^{k+2}}}{\equiv} \varphi\left(h^{(k)}(s)\right)+\sum_{k=1}^{q} h_{k+1}^{2} \beta^{\lambda} s^{k+1},
\end{array}
$$

where $\left\{\beta_{\lambda}\right\}_{1 \leq i \leq q}$ denotes the base of $\mathscr{H}$. Since the map $f_{\xi^{(k)}(s)+\eta_{k+1}^{j}(s)}$ is well defined globally on $M$ modulo $s^{k+2}$, we have

$$
P\left(\omega(s) \circ f_{\xi^{(k)}(s)+\eta_{k+1}^{j}(s)}\right) \underset{\bmod s^{k+2}}{\equiv} 0 .
$$

While the following relation holds.

$$
P\left(\varphi\left(h^{(k)}(s)\right)\right)=0 .
$$

From (6.22) and (6. 23), we have

$$
\begin{aligned}
& \bar{\partial}_{T^{\prime}}^{(1)}\left(\omega(s) \circ f_{\xi^{(k)}(s)+\eta_{k+1}^{j}(s)}\right)-\bar{\partial}_{T^{\prime}}^{(1)} \varphi\left(h^{(k)}(s)\right) \\
& +R_{2}\left(\omega(s) \circ f_{\xi^{(k)}(s)+\eta_{k+1}^{j}(s)}\right)-R_{2}\left(\varphi\left(h^{(k)}(s)\right)\right) \\
& +R_{3}\left(\omega(s) \circ f_{\xi^{(k)}(s)+\eta_{k+1}^{j}(s)}\right)-R_{3}\left(\varphi\left(h^{(k)}(s)\right)\right. \\
& \underset{\bmod s^{k+2}}{\equiv} 0 .
\end{aligned}
$$


From the assumption of the induction, we have

$$
\omega(s) \circ f_{\xi^{(k)}(s)+\eta_{k+1}^{j}(s)} \equiv \underset{\bmod s^{k+1}}{\equiv} \varphi\left(h^{(k)}(s)\right) .
$$

From (6.24) and (6.25), we have

$$
\bar{\partial}_{T^{\prime}}^{(1)}\left(\omega(s) \circ f_{\xi^{(k)}(s)+\eta_{k+1}^{j}(s)}-\varphi\left(h^{(k)}(s)\right)\right) \equiv \underset{\bmod s^{k+2}}{\infty} 0 .
$$

We shall put the homogeneous polynomials $h_{k+1}(s)$ and $\zeta_{k+1}(s)$ of degree $k+1$ as follows :

$$
h_{k+1}(s) \underset{\bmod s^{k+2}}{\equiv} \mathscr{L}\left(H_{T^{\prime}}^{(1)}\left(\omega(s) \circ f_{\xi^{(k)}(s)+\eta_{k+1}^{j}(s)}-\varphi\left(h^{(k)}(s)\right)\right)\right)
$$

and

$$
\zeta_{k+1}(s) \underset{\bmod s^{k+2}}{\equiv} \bar{\partial}_{T^{\prime}}^{*} N_{T^{\prime}}\left(\omega(s) \circ f_{\zeta^{(k)}(s)+\eta_{k+1}^{j}(s)}-\varphi\left(h^{(k)}(s)\right)-h_{k+1}(s)\right) .
$$

Then the equation (6.20) is satisfied (Hodge decomposition theorem).

We shall prove that the above formal power series $\sum_{k=1}^{\infty} h_{k}(s)$ and $\sum_{k=0}^{\infty}\left(\eta_{k+1}^{j}(s)+\zeta_{k+1}(s)\right)$ converge in \|\|$_{(m)}^{\prime}$-norm, to be introduced next, for sufficiently small $|s|$. To do this, we shall recall the following estimate (Propositions 3. 10 and 3. 11).

Proposition 3.10. The estimate

$$
\|\mu\|_{(m)}^{\prime \prime 2} \leqq\left\|\square_{T^{\prime}} \mu\right\|_{(m)}^{2}+\|\mu\|_{(m)}^{2}
$$

holds uniformly for all $\mu$ in $\Gamma\left(M, T^{\prime} \otimes\left({ }^{0} T^{\prime \prime}\right)^{*}\right)$ and for all non-negative integer $m$.

Proposition 3.11. The estimate

$$
\left\|N_{T^{\prime}} \mu\right\|_{(m)}^{\prime \prime} \leqq\|\mu\|_{(m)}
$$

holds uniformly for all $\mu$ in $\Gamma\left(M, T^{\prime} \otimes\left({ }^{\circ} T^{\prime \prime}\right)^{*}\right)$ and for all non-negative integer $m$, where $N_{T^{\prime}}$ denotes the Neumann operator for $\square_{T^{\prime}}$.

Let $\Gamma=\left\{\Gamma_{i}\right\}_{i \in \Lambda}$ be a chain where $\Gamma_{i}$ is a $T^{\prime}$-valued form on $V_{i}=\mathscr{V}_{i} \cap M$ and $\left\{W_{i}\right\}_{i \in \Lambda}$ be a refinement of $\left\{V_{i}\right\}_{i \in \Lambda}$. We define a semi-norm on $T^{\prime}$-valued chain as follows.

For each $\Gamma=\left\{\Gamma_{i}\right\}_{i \in A}$ in $C^{0}\left(V_{i}, T^{\prime}\right)$, we put 


$$
\|\Gamma\|_{(m)}^{2}=\sum_{i} \sum_{\alpha} \sum_{|\beta| \leq m} \int_{w_{i}} P_{\beta} \Gamma_{i}^{\alpha} \cdot \overline{P_{\beta} \Gamma_{i}^{\alpha}} d \mathscr{V}
$$

where we use the notations $C^{0}\left(V_{i}, T^{\prime}\right)$ for $T^{\prime}$-valued chains, by $\Gamma_{i}^{\alpha} \alpha=1, \ldots, n$ we denote $C^{\infty}$ functions on $V_{i}$ defined as follows;

$$
\Gamma_{i}=\sum_{r=1}^{n-1} \Gamma_{i}^{r} \bar{e}_{r}^{i}+\Gamma_{i}^{n} \xi
$$

where $\left\{\bar{e}_{r}^{i}\right\}_{1 \leq r \leq n-1}$ and $\xi$ are the system of moving frame of $T^{\prime} \mid V_{i}$ introduced in Lemma 6.7 and $d \mathscr{V}$ means the volume element on $V_{i}=\mathscr{V}_{i} \cap M$ induced by the diffeomorphism to a ball in $R^{2 n-1}$. And we set

$$
P_{\beta}=\partial^{\beta} / \partial x_{i_{1}}^{\beta_{1}} \ldots \partial x_{i_{l}}^{\beta_{l}}, \quad \sum_{k=1}^{l} \beta_{k}=\beta
$$

where $\left\{x_{i k}\right\}$ is a real coordinate system on $V_{i}$. With these notations we define a new norm \|\|$_{(m)}^{\prime}$ as follows: for each $\Gamma=\left\{\Gamma_{i}\right\}_{i \in A}$ in $C^{0}\left(V_{i}, T^{\prime}\right)$,

$$
\begin{aligned}
\|\Gamma\|_{(m)}^{2} & =\sum_{r}\left\|e_{r} \Gamma\right\|_{(m)}^{2} \\
& +\sum_{r}\left\|\bar{e}_{r} \Gamma\right\|_{(m)}^{2} \\
& +\sum_{r}\|\Gamma\|_{(m)}^{2}
\end{aligned}
$$

where by $e_{r} \Gamma$ we denote the $T^{\prime}$-valued function on $V_{i}$ defined as follows :

$$
e_{r} \Gamma=\sum_{\alpha=1}^{n-1} e_{r}^{i} \cdot \Gamma_{\alpha}^{i} \cdot \bar{e}_{\alpha}^{i}+e_{r}^{i} \cdot \Gamma_{n}^{i} \cdot \xi
$$

and we put

$$
\left\|e_{r} \Gamma\right\|_{(m)}^{2}=\sum_{i} \sum_{\alpha} \sum_{|\beta| \leq m} \int_{w_{i}} P_{\beta} e_{r}^{i} \Gamma_{i}^{\alpha} \cdot \overline{P_{\beta} e_{r}^{i} \Gamma_{i}^{\alpha}} d \mathscr{V}
$$

After this we fix these real coordinate and moving frames.

Now we shall prove the convergence. Consider the series:

$$
A(s)=b / 16 c \sum_{k=1}^{\infty}(c s)^{k} / k^{2},
$$

where $b$ and $c$ are constants to be determined later. Then we have

$$
A(s)^{n} \ll(b / c)^{n-1} A(s),
$$


where we write $a(t) \ll b(t)$ if $a(t)$ and $b(t)$ are power series $\sum_{k=0}^{\infty} a_{k} t^{k}$ and $\sum_{k=0}^{\infty} b_{k} t^{k}$ such that

$$
\left|a_{k}\right|<b_{k} .
$$

For each power series in $C^{0}\left(V_{i}, T^{\prime}\right)$, we set

$$
\|\Gamma(t)\|_{(m)}^{2}=\sum_{k=0}^{\infty}|| \Gamma_{k} \|^{\prime 2} t^{k}
$$

Then we have the relations: for any $\Gamma_{1}(t)$ and $\Gamma_{2}(t)$ in $C^{0}\left(V_{i}, T^{\prime}\right)$,

$$
\left\|\Gamma_{1}(t)+\Gamma_{2}(t)\right\|_{(m)}^{\prime} \ll\left\|\Gamma_{1}(t)\right\|_{(m)}^{\prime}+\left\|\Gamma_{2}(t)\right\|_{(m)}^{\prime}
$$

and

$$
\left\|\Gamma_{1}(t) \Gamma_{2}(t)\right\|_{(m)}^{\prime} \ll C_{m}^{\prime}|| \Gamma_{1}(t) \|\left._{(m)}^{\prime}|| \Gamma_{2}(t)\right|_{l_{(m)}} ^{\prime}
$$

hold.

In fact for any $\Gamma_{1}(t)=\sum_{k=0}^{\infty} \Gamma_{1, k} t^{k}$ and $\Gamma_{2}(t)=\sum_{k=0}^{\infty} \Gamma_{2 \cdot k} t^{k}$, we have $\left\|\Gamma_{1}(t)\right\|_{(m)}^{\prime}=\sum_{k=0}^{\infty}\left\|\Gamma_{1, k}\right\|_{(m)}^{\prime} t^{k}$ and $\left\|\left.\Gamma_{2}(t)\right|_{(m)} ^{\prime}=\sum_{k=0}^{\infty}\right\| \Gamma_{2 \cdot k} \|_{(m)}^{\prime} t^{k}$. Therefore from the relation: $\left\|\Gamma_{1 \cdot k}+\Gamma_{2 \cdot k}\right\|_{(m)}^{\prime} \leq\left\|\Gamma_{1 \cdot k}\right\|_{(m)}^{\prime}+\left.\right|_{1} ^{\prime} \Gamma_{2 \cdot k} \|_{(m)}^{\prime}$, we have (6. 27). Similary we have

$$
\Gamma_{1}(t) \cdot \Gamma_{2}(t)=\sum_{r=0}^{\infty}\left(\sum_{\substack{r=k+l \\ k, l \geq 0}} \Gamma_{1 \cdot k} \cdot \Gamma_{1 \cdot r}\right) t^{r}
$$

Wh le $\left\|\Gamma_{1 \cdot k} \cdot \Gamma_{1 \cdot \bullet}\right\|_{(m)}^{\prime} \leq C_{m}\left\|\Gamma_{1 \cdot k}\right\|_{(m)}^{\prime}|| \Gamma_{1 \cdot r} \|_{(m)}^{\prime} \quad$ since $m \geq n-1$. Therefore we have (6.28).

To prove that the formal power series $\sum_{k=0}^{\infty} h_{k+1}(s)$ and $\sum_{k=0}^{\infty}\left(\zeta_{k+1}(s)\right.$ $\left.+\eta_{k+1}^{j}(s)\right)$ converge, it suffices to see the following relation:

$$
\left\|\zeta_{k+1}(s)\right\|_{(m)}^{\prime} \ll A(s), \quad\left\|\eta_{k+1}^{j}(s)\right\|_{(m)}^{\prime} \ll A(s)
$$

and

$$
\left\|h_{k+1}(s)\right\|_{(m)}^{\prime} \ll 1 / 2 K A(s),
$$

where $K$ is a constant, whose existence we assert, such that $\left\|\bar{\partial}_{T^{\prime}}^{*} N_{T^{\prime}} \mu\right\|_{(m)}{ }_{(m)} \leq K|| \mu \|_{(m)}^{\prime}$ for all $\mu \in \Gamma\left(M, T^{\prime} \otimes\left({ }^{\circ} T^{\prime \prime}\right)^{*}\right)$ for all integer $k$ if $b$ and $c$ are sufficiently large. We set $b$ and $c$ sufficiently large so that the following estimate holds.

$$
\left\|\left(z_{j}(s)-z_{j}\right)\right\|_{(m+2)} \ll A(s) .
$$


By induction, we shall prove the relations (6.29) and (6.30). For $k=0$, it is easily seen that the relations (6.29) and (6.30) hold if $b$ and $c$ are sufficiently large. Assume $(6.29)_{k}$ and $(6.30)_{k}$ and let us prove $(6.29)_{k+1}$ and $(6.30)_{k+1}$.

From the relations $(6.16)$ and $\omega(s) \cdot f_{\zeta^{(k)}(s)}(X) \underset{\bmod s^{k+1}}{\equiv} \varphi\left(h^{(k)}(s)\right)$, we have the relation:

$$
\begin{aligned}
& \omega(s) \cdot f_{\xi^{(k)}(s)+\eta_{k+1}^{j}}(X)_{j}^{\alpha} \\
& \underset{\bmod s^{k+2}}{\equiv} \varphi\left(h^{(k)}(s)\right) \cdot\left(z_{j}^{\alpha}-z_{j}^{\alpha}(s)\right)+X\left(z_{j}^{\alpha}(s)-z_{j}^{\alpha}\right) \\
& +X \zeta^{(k)}(s)\left(z_{j}^{\alpha}(s)\right)+\varphi\left(h^{(k)}(s)\right) \zeta^{(k)}(s)\left(z_{j}^{\alpha}(s)\right) \\
& +X \eta_{k+1}^{j}(s) \text {. }
\end{aligned}
$$

We use the notations $\left(\omega(s) \cdot f_{\xi^{(k)}(s)+\eta_{k+1}^{j}(s)}(X) z_{j}^{\alpha}\right)^{k+1}$ for the homogeneous term of degree $k+1$. Then from (6.31), we have

$$
\begin{aligned}
& \left(\left(\omega(s) \cdot f_{\zeta^{(k)}(s)+\eta_{k+1}^{j}(s)}(X)\right) z_{j}^{\alpha}\right)^{k+1} \\
& \quad \equiv\left(\varphi\left(h^{(k)}(s)\right) \cdot\left(z_{j}^{\alpha}-z_{j}^{\alpha}(s)\right)\right)^{k+1} \\
& \quad+\left(X\left(z_{j}^{\alpha}(s)-z_{j}^{\alpha}\right)\right)^{k+1}+\left(X \zeta^{(k)}(s)\left(z_{j}^{\alpha}(s)-z_{j}^{\alpha}\right)\right)^{k+1} \\
& \quad+\left(\varphi\left(h^{(k)}(s)\right) \cdot \zeta^{(k)}(s)\left(z_{j}^{\alpha}(s)-z_{j}^{\alpha}\right)\right)^{k+1} \\
& \quad+X \eta_{k+1}^{j}(s)+\left(\varphi\left(h^{(k)}(s)\right) \zeta^{(k)}(s) z_{j}^{\alpha}\right)^{k+1}
\end{aligned}
$$

Therefore we have the relation:

$$
\begin{aligned}
& \left\|\left(\omega(s) \cdot f_{\xi^{(k)}(s)+\eta_{k+1}^{j}(s)}\right)^{k+1}\right\|_{(m)} \\
& \ll C_{m}\left\{\left\|\eta_{k+1}^{j}(s)\right\|_{(m)}^{\prime}\right. \\
& +\left\|\varphi\left(h^{(k)}(s)\right)\right\|_{(m)}\left\|\zeta^{(k)}(s)\right\|_{(m)}^{\prime} \\
& \left.+\left\|\varphi\left(h^{(k)}(s)\right)\right\|_{(m)}\left\|\left.\right|^{(k)}(s)\right\|_{(m)}^{\prime} \mid z_{j}^{\alpha}(s)-z_{j}^{\alpha} \|_{(m+2)}\right\},
\end{aligned}
$$

where for each element in $\Gamma\left(M, T^{\prime} \otimes\left({ }^{0} T^{\prime \prime}\right)^{*}\right)$ we use the norm introduced in $\S 3$. Moreover from the definition of $\eta_{k+1}^{j}(s)$ :

$$
\eta_{k+1}^{j}(s) \underset{\bmod s^{k+2}}{\equiv} \sum_{i}\left(\partial f_{j, i} / \partial z_{i}\right) \rho_{i} \sigma_{i, j},
$$

where $\quad \sigma_{i, j}=\left(\sigma_{i, j}^{\alpha}\right)_{1 \leq \alpha \leq n} \quad$ and $\quad \sigma_{i, j}^{\alpha}=z_{i}^{\alpha}(s)+\zeta^{(k)}(s)\left(z_{i}^{\alpha}(s)\right)-f_{i, j}^{\alpha}\left(z_{j}(s)\right.$ $\left.+\zeta^{(k)}(s)\left(z_{j}(s)\right), s\right)$, we have the following estimate by an induction.

$$
\left\|\eta_{k+1}^{j}(s)\right\|_{(m)}^{\prime} \ll K_{1} A(s)^{2},
$$

where $\mathrm{K}_{1}$ is independent of $k$.

In fact, $\sigma_{i, j}$ being a homogeneous polynomial of degree $k+1$, we 
have the relation:

$$
\begin{aligned}
\sigma_{i, j}^{\alpha} & =\left(z_{i}^{\alpha}(s)-f_{i, j}^{\alpha}\left(z_{j}(s)+\zeta^{(k)}(s)\left(z_{j}(s)\right), s\right)\right)^{k+1} \\
& +\left(\zeta^{(k)}(s)\left(z_{i}^{\alpha}(s)-z_{i}^{\alpha}\right)\right)^{k+1} \\
& =\left(z_{i}^{\alpha}(s)-f_{i, j}^{\alpha}\left(z_{j}(s)+\zeta^{(k)}(s)\left(z_{j}(s)-z_{j}+\zeta^{(k)}(s) z_{j}, s\right)\right)^{k+1}\right. \\
& +\left(\zeta^{(k)}(s)\left(z_{i}^{\alpha}(s)-z_{i}^{\alpha}\right)\right)^{k+1} .
\end{aligned}
$$

We use the notation:

$$
\begin{aligned}
& z_{i}^{\alpha}(s)-f_{i, j}^{\alpha}\left(z_{j}(s)+u+v, s\right) \\
& =\sum_{\substack{I, L, k \\
|I I| \neq 0 \text { or }|L| \neq 0}} A_{I, L, k}^{\alpha} u^{I} v^{L} s^{k} .
\end{aligned}
$$

Then from (6.34) we have the relation:

$$
\begin{aligned}
& \sigma_{i, j}^{\alpha}=\left(\sum_{\beta} A_{0,1,0}^{\alpha}\left(\zeta^{(k)}(s) z_{j}^{\beta}\right)\right. \\
& \left.+\sum_{\substack{I_{i} L_{i}, k \\
(I I,|L|, k) \neq(0,1,0)}}^{\beta} A_{I, L, k}\left(\zeta^{(k)}(s)\left(z_{i}(s)-z_{i}\right)\right)^{I}\left(\zeta^{(k)}(s) z_{j}\right)^{L} s^{k}\right)^{k+1} \\
& +\left(\zeta^{(k)}(s)\left(z_{i}^{\alpha}(s)-z_{i}^{\alpha}\right)\right)^{k+1}
\end{aligned}
$$

where by $I$ and $L$ we denote multiindices. $\zeta^{(k)}(s) z_{j}^{\beta}$ being a polynomial of degree $k=1$, we have

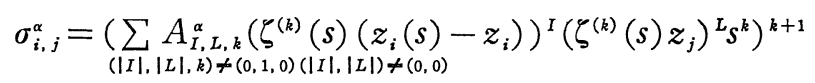

$$
\begin{aligned}
& +\left(\zeta^{(k)}(s)\left(z_{i}^{\alpha}(s)-z_{i}^{\alpha}\right)\right)^{k+1} .
\end{aligned}
$$

We may assume the relations

$$
\left\|\rho_{i} A_{I, L, k}^{\alpha}\right\|_{(m)}^{\prime} \ll B_{0}^{\prime} \cdot B_{1}^{\prime|I|+|L|+k}
$$

for any $I, L$ and $k$.

Similarly we have

$$
\left\|\left(\partial f_{j, i} / \partial z_{i}\right) \rho_{i}\left(A_{I, L, k}\right)\right\|_{(m)}^{\prime} \ll B_{0} \cdot B_{1}^{|I|+|L|+k}
$$

for any $I, L$ and $k$. We may assume

$$
\|s\|_{(m)}^{\prime} \ll A(z) \text { as a chain. }
$$

So from (6.35) and an induction, we have the relation:

$$
\begin{aligned}
& \left\|\left(\partial f_{j, i} / \partial z_{i}\right) \rho_{i} \sigma_{i, j}\right\|_{(m)}^{\prime} \\
& \ll C_{1}|A(z)|^{2}+\sum_{\substack{|I|, \mid \\
(|I|,|L|, k) \neq(0,1,0)}} B_{L \mid+(0,0)} \cdot B^{|I|+|L|+k} A(s)^{2|I|+|L|+k}
\end{aligned}
$$

now the relation $2|I|+|L|+k \geq 2$ holds. So we have the relations 


$$
(A(s))^{2 I+|L|+k} \ll(b / c)^{2 I+|L|+k-2} A(s)^{2} .
$$

From (6.36) and (6.37), we have the relation:

$$
\begin{aligned}
& \left.\|\left(\partial f_{j, i} / \partial z_{i}\right) \rho_{i} \sigma_{i, j}\right) \|_{(m)}^{\prime}
\end{aligned}
$$

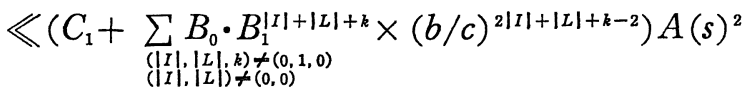

$$
\begin{aligned}
& \ll C_{2} A(s)^{2} \text {, }
\end{aligned}
$$

if $c$ are sufficiently large. So we have

$$
\left\|\eta_{k+1}^{j}(s)\right\|_{(m)}^{\prime} \ll K_{1} A(s)^{2}
$$

$\varphi(t)$ being a convergent power series, we have the relation:

$$
\left\|\varphi_{l}\right\|_{(m)}^{\prime} \leq B_{2} B_{3}^{l}
$$

for any $l$, where $\varphi(t)=\sum_{l \geq 1} \varphi_{l} t^{l}$. So we have the relation:

$$
\begin{aligned}
& \left\|\varphi\left(h^{(k)}(s)\right)\right\|_{(m)} \\
& \ll\left\|\varphi\left(h^{(k)}(s)\right)\right\|_{(m)}^{\prime} \\
& \ll \sum_{\sum_{l}=B_{2} B_{3}^{l}\left(h^{(k)}(s)\right)^{l}} \\
& \ll \sum_{l \geq 1} B_{2} \cdot B_{3}^{l} \cdot(b / c)^{l-1} A(s) \\
& \ll K_{2} A(s) .
\end{aligned}
$$

From (6.33), (6.38) (6.39) and the construction of $h_{k+1}(s)$, we have

$$
\begin{aligned}
\left\|h_{k+1}(s)\right\|_{(m)}^{\prime} & \ll K^{\prime} A(s)^{2} \\
& \ll K^{\prime} b / c A(s) \\
& \ll 1 / 2 K A(s)
\end{aligned}
$$

if $c$ is sufficiently large, where $K^{\prime}$ is independent of $k$. Similarly we have

$$
\left\|\zeta_{k+1}(s)\right\|_{(m)}^{\prime} \ll A(s) .
$$

Therefore $\sum_{k=0}^{\infty} h_{k+1}(s)$ and $\sum_{k=0}^{\infty}\left(\zeta_{k+1}(s)+\eta_{k+1}^{j}(s)\right)$ converge in \|\|$_{(m)}^{\prime}$ norm. By Sobolev lemma, we have that they are in $C^{2}$-class. So we have our main Theorem. 


\section{References}

[1] Akahori, T., Intrinsic formula for Kuranishi's $\bar{\partial}_{b}^{\varphi}$, to appear in Publ. RIMS.

[2] Folland, G. B. and Stein, E. M., Estimates for the $\bar{\partial}_{b}$ Complex and Analysis on the Heisenberg Group. Comm. Pure Appl. Math., 27 429-522.

[3] Kohn, J.J., Boundaries of Complex Manifolds. Proceedings of the Conference on Complex Analysis, Minneapolis, Springer, 1955.

[4] Kuranishi, M., Deformations of isolated singularities and $\vec{\partial}_{b}$. Preprint, Columbia Univ., 1973.

[5] Milnor, J., Singular Points of Complex Hypersurfaces, Ann. of Math. Studies 61, Princeton, N. J., 1968.

[6] Rossi, H., Attaching Analytic spaces to an Analytic Space along a Pseudo-concave Boundary, Proceeding of Conference on Complex Analysis, Minneapolis, Springer, (1965).

[7] Tanaka, N., A Differential Geometric Study on Strongly Pseudo-convex Manifolds, Lectures in Math., Dep. of Maht. Kyoto Univ.

[8] Kodaira, K. and Spencer, D.C., On deformations of complex analytic structures, I and II. Ann Math.67, (1958a), 328-466. 
